\title{
About the effects of polarising optics on lidar signals and the $\Delta 90$ calibration
}

\author{
Volker Freudenthaler \\ Fakultät für Physik, Meteorologisches Institut, Ludwig-Maximilians-Universität, Theresienstrasse 37, \\ 80333 Munich, Germany
}

Correspondence to: Volker Freudenthaler (volker.freudenthaler@lmu.de)

Received: 4 November 2015 - Published in Atmos. Meas. Tech. Discuss.: 11 February 2016

Revised: 25 July 2016 - Accepted: 27 July 2016 - Published: 31 August 2016

\begin{abstract}
This paper provides a model for assessing the effects of polarising optics on the signals of typical lidar systems, which is based on the description of the individual optical elements of the lidar and of the state of polarisation of the light by means of the Müller-Stokes formalism. General analytical equations are derived for the dependence of the lidar signals on polarisation parameters, for the linear depolarisation ratio, and for the signals of different polarisation calibration setups. The equations can also be used for the calculation of systematic errors caused by nonideal optical elements, their rotational misalignment, and by non-ideal laser polarisation. We present the description of the lidar signals including the polarisation calibration in a closed form, which can be applied for a large variety of lidar systems.
\end{abstract}

\section{Introduction}

The purpose of atmospheric depolarisation measurements with lidar, first described by Schotland et al. (1971), is mainly to discern between more or less depolarising scatterers. The discrimination of ice and water clouds was the main focus in the beginning. Sassen $(1991,2005)$ gives an overview about the early work related to that. Aerosol and their interaction with clouds have become more important in the last decade because of their insufficiently understood direct and indirect roles in the feedback mechanisms of climate change (Boucher et al., 2013). Multi-wavelength lidar measurements including the depolarisation ratio can be used to discern aerosol types (Sugimoto et al., 2002; Sugimoto and Lee, 2006; Ansmann et al., 2011; Burton et al., 2014; Groß et al., 2014) and to retrieve micro-physical aerosol properties by means of inversion algorithms (Müller et al., 1999; Ansmann and Müller, 2005; Gasteiger et al., 2011; Veselovskii et al., 2013; Böckmann and Osterloh 2014; Müller et al., 2014).

Published by Copernicus Publications on behalf of the European Geosciences Union. 
Pérez-Ramírez et al. (2013) show the impact of systematic errors of the lidar data on the retrieval of micro-physical particle properties. The additional measurement of the linear or circular depolarisation ratio improves the retrievals (Böckmann and Osterloh, 2014; Gasteiger and Freudenthaler, 2014). But the depolarisation ratios are often derived from lidar measurements assuming more or less ideal lidar setups neglecting the effects of small system misalignments and of non-ideal optical elements on the polarisation, which can lead to considerable errors in the retrieved depolarisation ratio (Reichardt et al., 2003; Alvarez et al., 2006; Freudenthaler et al., 2009; Mattis et al., 2009). According to Chipman (2009a, chap. 15.27), one of the primary difficulties in performing accurate polarisation measurements is the systematic error due to non-ideal polarisation elements. Most inclined optical surfaces and optical coatings on beam splitters are polarising; therefore, all lidars must be considered "incomplete light-measuring polarimeters" (Chipman, 2009a), even if they are not intended to measure the depolarisation ratio.

As model calculations of aerosol scattering properties advance (Nousiainen et al., 2011; Kahnert et al., 2014), the modellers need accurate measurements with small errors and reliable error bars in order to verify and improve their models. In order to estimate the uncertainties and to improve the measurements, we have to find the error sources. The usual way to do this is to compare the measurements with a model and to investigate the deviations. The only reliable atmospheric model for comparison is the model of the molecular linear depolarisation ratio $\delta_{m}$ (Behrendt and Nakamura 2002; Freudenthaler et al., 2015). But the actually measured values $\delta_{m}{ }^{*}$ of the very small real $\delta_{m}$ (on the order of 0.004 ) are usually a number of times higher, which makes it difficult to use them for the calibration with a simple model as $\delta_{m}{ }^{*}$ $=A \delta+B$ (Sassen and Benson 2001; Reichardt et al., 2003) (see also Sect. S9 in the Supplement). At present, polarisation calibration techniques of lidars are often not accurate enough to sufficiently determine the two parameters $A$ and $B$, and actually, as we will show in the following, the model itself is insufficient. But how accurate do we have to be? How accurate can we be? Which are the critical parts and adjustments? How can setups be improved with minimal costs and complexity, and how can existing lidar systems be checked? To answer these questions, we need a better model for the lidar setup, which is complete and flexible enough to be applied to a variety of lidar systems and can describe various calibration techniques.

Astronomical polarisation measurement setups are very similar to lidar setups. Elaborate theoretical and experimental investigations of the influence of polarising optics and corresponding corrections for astronomical telescopes and detection optics using the theory of polarimetry and ellipsometry (see Azzam, 2009; Chipman, 2009a) can be found quite frequently in the literature (Skumanich et al., 1997; Socas-Navarro et al., 2011; Breckinridge et al., 2015). Although the usefulness of a lidar with polarisation diversity had been realised early (Pal and Carswell 1973), the need for a complete description with the Müller-Stokes formalism was, to our knowledge, first expressed by Anderson (1989) but focused only on the atmospheric scattering process. Instrumental aspects including some error calculations have been included by Beyerle (1994), Cairo et al. (1999), Biele et al. (2000), Behrendt and Nakamura (2002), Reichardt et al. (2003), Alvarez et al. (2006), Del Guasta et al. (2006), Hayman and Thayer (2009), Mattis et al. (2009), Freudenthaler et al. (2009), Hayman (2011), Hayman and Thayer (2012), David et al. (2013), Geier and Arienti (2014), Di et al. (2015), and Volkov et al. (2015). The errors mainly considered are the diattenuation of the receiver optics (see Sect. 2.2), 
the cross-talk of the polarising beam splitter, non-ideal characteristics of the calibration, and rotational misalignment of polarising components.

In this work we describe lidar setups from the laser to the detector by means of the Stokes-Müller formalism (Chipman $2009 \mathrm{~b}$ ) including the transmitter and receiver optics. The Stokes vector describes the flux and the state of polarisation of the light, and the Müller matrices describe how optical elements change the Stokes vector. We develop equations for the two signals of a polarisation-sensitive lidar and for the signals of the polarisation calibration, which are necessary to retrieve the linear depolarisation ratio and the total lidar signal, using different calibration techniques and lidar setups. In order to enable the evaluation of the final errors and to analyse their dependencies on certain optical parameters or misalignments of individual optical elements, we derive first the full equations and then try to find more simple analytical formulations neglecting minor error sources to get an overview of the main critical parameters. For this we neglect the polarisation effects of lenses and of telescope mirrors with small incidence angles of the light beam (Seldomridge et al., 2006; Clark and Breckinridge 2011). Although not considered here, $90^{\circ}$ folding mirrors as in Newtonian-type telescopes (Breckinridge et al., 2015; Di et al., 2015) and stress birefringence in windows and lenses or unfavourable coatings might cause severe polarisation effects. This requires further investigation. In general, errors caused by a light beam which is divergent or inclined towards the optical axis are not discussed here; this means the light beams are assumed to be either perfectly parallel before and after polarisation optics, or that an optical element is insensitive to the incident angle regarding polarisation.

Basic information about the polarisation topics can be found in Goldstein (2003), Clarke (2009), and in the chapters by Azzam (2009), Bennett (2009a, b), and Chipman (2009b, a) of the 3rd edition of the Handbook of Optics (Bass, 2009). The authors of these chapters follow the Muller Nebraska convention (Muller, 1969) for the definition of signs and directions regarding, for example, the coordinate system (see Supplement Sect. S1), as we do in this work.

Most of the lidar setups for depolarisation measurement reported in the literature are explicable with the schematic in Fig. 1, in which the individual parts of a lidar system are grouped in modules, which are in general describable by Müller matrices of combinations of diattenuators, retarders, and rotators (see Sect.2.2). The setup in Fig. 1 can be described with Eq. (1).

$$
\boldsymbol{I}_{T, R}=\eta_{T, R} \mathbf{M}_{T, R} \mathbf{C} \mathbf{M}_{O} \mathbf{F} \mathbf{M}_{E} \boldsymbol{I}_{L}
$$

Symbols for Müller matrices are bold (M), vectors are bold and italic $(\boldsymbol{I})$, and variables italic $(I)$. The laser beam with Stokes vector $\boldsymbol{I}_{L}$ is expanded and directed towards the atmosphere with backscatter matrix $\mathbf{F}$ by the emitter module with Müller matrix $\mathbf{M}_{E}$. The backscattered photons are received by the telescope with a subsequent collimation lens and dichroic beam splitters in the receiver optics module $\mathbf{M}_{O}$. A polarisation calibrator with Müller matrix $\mathbf{C}$ is placed here before the polarising beam splitter cube (10) with Müller matrices $\mathbf{M}_{T}$ for the transmitted and $\mathbf{M}_{R}$ for the reflected path, their opto-electronic gains $\eta_{T, R}$, and the final Stokes vectors $\boldsymbol{I}_{T, R}$ at the detectors. The opto-electronic gains $\eta_{T, R}$ include the attenuation of all non-polarising optical elements such as neutral density and bandpass filters, the efficiency of the detectors, and the amplification of the electronic system. The scattering volume $\mathbf{F}$ can be at any distance from the lidar 
(lidar range) because we assume that the extinction in the range between the lidar and the scattering volume $\mathbf{F}$ is polarisation-independent and that signal contributions due to forward or multiple scattering in this range can be neglected. Therefore we neglect all lidar range dependencies in the following equations. We also do not consider rangedependent effects such as the overlap function and the range-dependent transmission of interference filters and dichroic beam splitters, which is caused by the range-dependent incident angles on the optics.
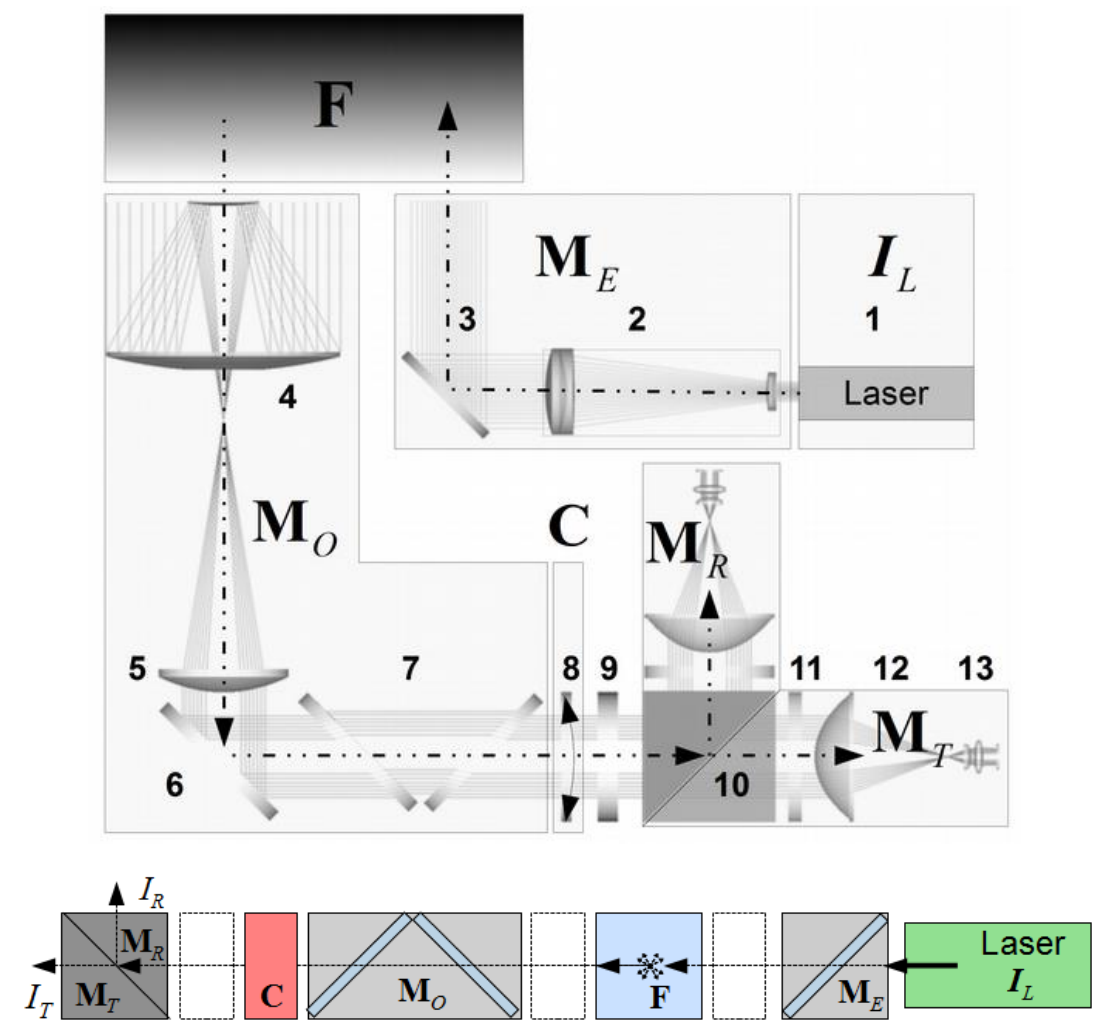

Figure 1. Top: exemplary depolarisation lidar setup with laser 1, beam expander 2, steering mirror 3, receiving telescope 4, collimator 5, folding mirror 6 , dichroic beam splitters 7 , a rotating element for polarisation calibration 8 , interference filter 9 , and polarising beam splitter cube 10 (PBS, polarising beam splitter). The neutral density filters and cleaning polarisers 11 , detector optics 12, and the detectors 13 . The system can be subdivided in functional blocks which can be described with the Stokes-Müller formalism: $\boldsymbol{I}_{L}$ is the Stokes vector of the laser source, $\mathbf{M}_{E}$ is the Müller matrix of the laser emitter optics, $\mathbf{F}$ of the atmospheric backscattering volume including depolarisation, $\mathbf{M}_{O}$ includes receiver optics as beam splitters, $\mathbf{C}$ is the calibrator, and $\mathbf{M}_{T, R}$ is the polarising beam splitter including the detector optics for the transmitted $(T)$ and reflected $(R)$ optical branches. Bottom: simplified schematic of the setup.

Various lidar systems employ different calibration techniques with calibrating devices with Müller matrix $\mathbf{C}$ at different places in the optical setup, with the respective equations: 
before the polarising beam splitter

before the receiver optics

behind the laser emitter optics

before the laser emitter optics

$$
\boldsymbol{I}_{S}=\eta_{S} \mathbf{M}_{S} \mathbf{C} \mathbf{M}_{O} \mathbf{F} \mathbf{M}_{E} \boldsymbol{I}_{L}
$$

In the following we list just a few examples from the literature with sufficient description of their calibration technique. Pal and Carswell (1973) used three telescopes with Glan-Thompson prisms in the receiver optics (Eq. 2) at 0, 45, and $90^{\circ}$ orientation with respect to the laser polarisation to determine the first three Stokes parameters of the scattered light and calibrated them by mechanically switching all polarisers to $0^{\circ}$ orientation. Houston and Carswell (1978) extended this setup by a fourth telescope with a $\lambda / 4$ plate to measure all four Stokes parameters, with the same calibration technique as before. The relative polarisation sensitivity of the CALIOP lidar on CALIPSO (Winker et al., 2009) is calibrated with a pseudo-depolariser before the polarising beam splitter (Hunt et al., 2009), which is described by Eq. (2). Del Guasta et al. (2006) calibrate the gain ratio $\eta_{R} / \eta_{T}$ of their polarimetric lidar with an unpolarised light source before the polarising beam splitter (Eq. 2) and determine the receiving optics Müller matrix $\mathbf{M}_{O}$ with a linearly polarised light source and rotating the receiving optics, which corresponds to Eq. (3) with a mechanical rotation matrix C. Similar rotation calibration before the polarising beam splitter is applied with RALI (Nemuc et al., 2013) and the Raymetrics LR331D400 (Bravo-Aranda et al., 2013) with a mechanical rotation $\Delta 90$ calibration (see Sect. 5), and with a $\lambda / 2$ plate rotation in the MULIS (Freudenthaler et al., 2009) and the Cloud Physics Lidar (McGill et al., 2002; Liu et al., 2004). A sheet polariser at $45^{\circ}$ is used before the polarising beam splitter in the AD-Net lidars (Shimizu et al., 2004). Mechanical rotation before the receiving optics (Eq. 3) is employed for the DLR HSRL (Esselborn et al., 2008), for POLIS (Freudenthaler et al., 2009), and by Nisantzi et al. (2014). For the McMurdo lidar (Snels et al., 2009) and the PollyXT (Engelmann et al., 2015) a linear polariser is used before the receiving optics. An unpolarised light source before the receiver telescope is used by Mattis et al. (2009). Spinhirne et al. (1982) use a $\lambda / 2$ plate for polarisation rotation in the output beam (Eq. 4). The HSRL-1 (Hair et al., 2008) and HSRL-2 (Burton et al., 2015) as well as David et al. (2012) use a $\lambda / 2$ plate as rotation calibrator before some parts of the emission optics (Eq. 5). Roy et al. (2011) and Cao et al. (2010) use a $\lambda / 2$ plate before the emitter optics (Eq. 5), but they switch the plane of emitted polarisation continually between horizontal and vertical and calculate the linear depolarisation ratio from the geometric mean of both measurements, which makes a separate calibration unnecessary. However, the equations of this work can still be used for the error analysis. Polarisation switching between laser pulses and with only one detection channel is done by Platt (1977) with mechanical rotation of the receiver optics, by Eloranta and Piironen (1994) with a $\lambda / 2$ plate after the emitter optics (Eq. 4), by Seldomridge et al. (2006) with a nematic liquid crystal before the polarising beam splitter (Eq. 2), and by Flynn et al. (2007) with a $\lambda / 2$ plate before the emitter optics (Eq. 5). Although the explicit equations in this work consider only one variable polarising element (i.e. the calibrator), the equations for more complex lidar setups as with a polarising beam splitter and a $\lambda / 4$ plate in the common emitter/receiver path (Eloranta 2005; David et al., 2013) or with different variable polarisation elements in the emitter/receiver path (Kaul et al., 2004; Hayman et al., 2012; 
Volkov et al., 2015) can be constructed with the equations provided in this work. Snels et al. (2009) present an overview of some potential error sources and other existing polarisation calibration techniques including calibration with assumed known depolarisation from molecules (“clear sky”) or clouds with spherical particles.

The equations presented in this work can be used for the design of lidar systems, especially for the determination of the requirements for certain components in order to achieve the desired measurement accuracy, for the analysis of the performance of existing lidar systems by means of different calibration setups, and for the final error calculation with respect to the polarisation characteristics.

One of the main uncertainties is the orientation of the plane of polarisation of the laser beam (angle $\alpha$ ) with respect to the orientation of the polarising beam splitter (briefly laser rotation) because first, the plane of polarisation of the laser might be determined not only by the orientation of the Pockels cell in the laser cavity but also by the orientation of the crystals for second and third harmonics generation and by the harmonic separation beam splitters. Second, the laser and emitter optics are often mounted on a separate optical breadboard, which might be rotated with respect to the receiver breadboard. Furthermore, laser manufacturers usually provide neither an indication of the accuracy of the orientation nor an accurate mechanical reference for it. The orientation cannot be measured easily, and finally the orientation can change with time and environmental conditions. We take into account that in lidar labs it is usually not possible to perform elaborate and accurate measurements as in an optical lab equipped for ellipsometric measurements. Therefore we want to use simple tools and as few as possible measurements - at best with the tools which we already use for the standard depolarisation measurements.

Some optical parts can be made almost ideal and some misalignments can be made very small so that they become negligible. For these cases often much simpler equations can be derived, which show the residual influence of the other non-ideal parts, and which can be used directly in lidar retrieval algorithms. It becomes also clear in which cases corrections are not possible, when additional measurements with simple setups can help to retrieve the properties of the disturbing parts, and where one has to be careful in the design of a lidar system to avoid non-correctable errors. We want to find the setups and calibrators, with which the calibration can be measured with the least errors, and we want equations to assess the final uncertainties in the retrieved lidar products. Setups with $90^{\circ}$ separated limit stops can be made very accurate $\left(<0.1^{\circ}\right)$ by means of working machines. Motorised holders with sufficient resolution and accuracy are commercially available. An example for an almost ideal part is the linear polariser. Polarising sheet filters are available with high extinction, well specified by manufacturers. They are relatively insensitive to the incident angle, work over a sufficiently large wavelength range, and are thin, which means that they can be placed even in already existing lidar systems with little space for additional optics. Additionally, they are available in large size at an affordable price - in contrast to crystal polarisers and wave plates, and thus they can also be placed before the telescope. Wave plates and circular polarisers made of plastic sheets are usually not as well specified concerning their phase shift, acceptance angle, and wavelength range. For other places, which require only small diameters, true zero-order $\lambda / 2$ plates can be used. 
Since the atmosphere is not stable and the laser power might change between two consecutive measurements, the absolute signals change. But if we use the ratios of the cross and parallel signals, which only change with the atmospheric polarisation parameter $a$, we can easily find atmospheric situations which introduce negligible errors in the calculations. Therefore we only use signal ratios for the calibrations.

Most of the problems can probably be solved with a much smaller theoretical framework. But then often questions arise as to how the one or other misalignment, rotation, additional retardance, or diattenuation would influence the final results. The impotence of less extended formulations to answer these questions will always leave an uncomfortable uncertainty. This work is an attempt to provide the tools to answer some of these questions, with the disadvantage of being rather extended.

Section 2 provides a simplified example as an introduction and preparation for Sect. 3, where we introduce the concepts and parameters which are necessary to formulate the equations in such a general way that they can be applied to a large variety of lidar systems. In order to generalise and to simplify the expressions, several binary parameters are introduced in the equations, which enable us to describe orthogonal orientations of individual elements with just one expression and which reduce the number of equations considerably. In Sect. 4 we develop the general equations for the lidar signals of normal atmospheric measurements (standard measurements in the following) and for the linear depolarisation ratio. In Sect. 5 we introduce the general concept of the $45^{\circ}$ and $\Delta 90$ calibrations, which is then applied in Sect. 6 to 10 for different calibrators and in the subsections for different positions of the calibrators in the emitter-receiver optics. We include the following types of calibrators: unpolarised light (Sect. 6), which has to be inserted by an additional light source or diffuser and has therefore some disadvantages; the mechanical and $\lambda / 2$ plate rotator (Sect. 7); the linear polariser (Sect. 8), which can be easily included in existing systems; the $\lambda / 4$ plate (Sect. 9), which can also be used to determine the amount of circular polarisation; and the circular polariser (Sect. 10). General purpose equations used in several sections are shifted to the appendices, and common equations or concepts, which can also be found in standard text books, are collected in the supplement in order to show their form with the variables used in this work.

\section{The basic Müller-Stokes representation of lidar signals with polarisation}

In this chapter we use a simple example of Fig. 1, described with Eq. (2), to introduce some basic concepts. It contains a calibrator $\mathbf{C}$ before the polarising beam splitter and neglects the polarising effects of the receiver optics $\mathbf{M}_{O}$, i.e.

$$
\boldsymbol{I}_{T, R}=\eta_{T, R} \mathbf{M}_{T, R} \mathbf{C F I} \boldsymbol{I}_{L}
$$

The total power $I_{L}$ and the state of polarisation of horizontally linearly polarised laser light are represented by the Stokes vector

$$
\boldsymbol{I}_{L}=I_{L}\left(\begin{array}{l}
1 \\
1 \\
0 \\
0
\end{array}\right)
$$


The magnitude $I_{L}$ of the Stokes vector is the total light beam intensity. It is directly measurable with a light detector for the flux of photons. Because a lidar includes optics as telescope and lenses, which change the diameter or focus the light beam, here the colloquial intensity means the radiant flux or radiant energy per unit time. However, the finally measured quantities are the electronic signals $I_{T}$ and $I_{R}$ of the detectors in the transmitted and reflected paths. We use flux, intensity and signal alternatively, depending on the context.

\subsection{Depolarising atmospheric aerosol}

Müller matrices describe the linear interaction between polarised light and an optical system (optical elements or medium). For any input, represented as a Stokes vector, the Müller matrix produces a unique output, in the form of another Stokes vector. For the backscattering of a volume of randomly oriented, non-spherical particles with rotation and reflection symmetry the Müller matrix F can be written as (van de Hulst 1981; Mishchenko and Hovenier 1995; Mishchenko et al., 2002)

$\mathbf{F}=\left(\begin{array}{cccc}F_{11} & 0 & 0 & 0 \\ 0 & F_{22} & 0 & 0 \\ 0 & 0 & -F_{22} & 0 \\ 0 & 0 & 0 & F_{44}\end{array}\right)=F_{11}\left(\begin{array}{cccc}1 & 0 & 0 & 0 \\ 0 & a & 0 & 0 \\ 0 & 0 & -a & 0 \\ 0 & 0 & 0 & 1-2 a\end{array}\right)$

with the polarisation parameter $a$ (Chipman 2009b; Eq. 93)

$a=\frac{F_{22}}{F_{11}}$

and

$F_{44}=F_{11}-2 F_{22}=F_{11}(1-2 a)$

Note that in some literature (Flynn et al., 2007; Gimmestad, 2008; Roy et al., 2011; Gasteiger and Freudenthaler, 2014) the de-polarisation parameter $d=(1-a)$ is used, and in Borovoi et al. (2014) $d$ is called polarisation parameter. In Volkov et al. (2015) $e=a$ (for randomly oriented particles) is called sphericity index. However, in this work we use the polarisation parameter $a$ for the reason of brevity, which is the fraction of the backscattered light that maintains the emitted linear polarisation.

The matrix $\mathbf{F}$ in Eq. (8) describes a pure depolariser $\mathbf{M}_{\Delta}$ (Lu and Chipman 1996), but including a mirror reflection $\mathbf{M}_{M}$ for the backscattering direction, with the backscatter coefficient $F_{11}$. 
$\mathbf{F}=\mathbf{M}_{M} \mathbf{M}_{\Delta}=F_{11}\left(\begin{array}{cccc}1 & 0 & 0 & 0 \\ 0 & 1 & 0 & 0 \\ 0 & 0 & -1 & 0 \\ 0 & 0 & 0 & -1\end{array}\right)\left(\begin{array}{cccc}1 & 0 & 0 & 0 \\ 0 & a & 0 & 0 \\ 0 & 0 & a & 0 \\ 0 & 0 & 0 & 2 a-1\end{array}\right)$

$F_{11}$ and $a$ are the only range-dependent parameters in all the following equations. The volume linear depolarisation ratio $\delta$ of the scattering volume, which contains particles and air molecules, can be written as (Mishchenko and Hovenier 1995)

$\delta=\frac{F_{11}-F_{22}}{F_{11}+F_{22}}=\frac{1-a}{1+a} \Rightarrow a=\frac{1-\delta}{1+\delta}$

The Stokes vector $\boldsymbol{I}_{\text {in }}$ of horizontally linearly polarised light $\boldsymbol{I}_{L}$ reflected by the atmosphere $\mathbf{F}$ and incident in the receiving optics is

$\boldsymbol{I}_{\text {in }}=\mathbf{F} \boldsymbol{I}_{L}=F_{11}\left(\begin{array}{cccc}1 & 0 & 0 & 0 \\ 0 & a & 0 & 0 \\ 0 & 0 & -a & 0 \\ 0 & 0 & 0 & 1-2 a\end{array}\right) I_{L}\left(\begin{array}{l}1 \\ 1 \\ 0 \\ 0\end{array}\right)=F_{11} I_{L}\left(\begin{array}{l}1 \\ a \\ 0 \\ 0\end{array}\right)$

\subsection{Optical parts: diattenuator with retardation}

All other optical elements in the lidar receiver can be described as a combination of diattenuators and retarders (Lu and Chipman, 1996) (retarding diattenuators; Eq. 14). Often a polarising beam splitter cube is used for splitting in transmitted and reflected components polarised parallel and perpendicular with respect to the laser polarisation. But also polarising or even non-polarising beam splitter plates with subsequent polarisation filters (analysers) can be used. All of them and combinations of them can be described with the Müller matrix of a polarising beam splitter (PBS) (Pezzaniti and Chipman 1994), considering the remarks in Sect. S4. The matrix of the transmitting part is

$\mathbf{M}_{T}=\frac{1}{2}\left(\begin{array}{cccc}T_{T}^{p}+T_{T}^{s} & T_{T}^{p}-T_{T}^{s} & 0 & 0 \\ T_{T}^{p}-T_{T}^{s} & T_{T}^{p}+T_{T}^{s} & 0 & 0 \\ 0 & 0 & 2 \sqrt{T_{T}^{p} T_{T}^{s}} \cos \Delta_{T} & 2 \sqrt{T_{T}^{p} T_{T}^{s}} \sin \Delta_{T} \\ 0 & 0 & -2 \sqrt{T_{T}^{p} T_{T}^{s}} \sin \Delta_{T} & 2 \sqrt{T_{T}^{p} T_{T}^{s}} \cos \Delta_{T}\end{array}\right)=$

$=T_{T}\left(\begin{array}{cccc}1 & D_{T} & 0 & 0 \\ D_{T} & 1 & 0 & 0 \\ 0 & 0 & Z_{T} \mathrm{c}_{T} & Z_{T} \mathrm{~s}_{T} \\ 0 & 0 & -Z_{T} \mathrm{~s}_{T} & Z_{T} \mathrm{c}_{T}\end{array}\right)$ 
with the intensity transmission coefficients (transmittance) for light polarised parallel $\left(T^{\mathrm{p}}\right)$ and perpendicular $\left(T^{6}\right)$ to the plane of incidence of the PBS, the diattenuation parameter $D_{T}$, and the average transmittance $T_{T}$, i.e. for unpolarised light. $\Delta_{T}$ is the difference of the phase shifts of the parallel and perpendicular polarised electrical fields (retardance) according to the Muller Nebraska convention (Muller 1969).

$T_{T}=\frac{T_{T}^{p}+T_{T}^{s}}{2}, D_{T}=\frac{T_{T}^{p}-T_{T}^{s}}{T_{T}^{p}+T_{T}^{s}}, Z_{T}=\frac{2 \sqrt{T_{T}^{p} T_{T}^{s}}}{T_{T}^{p}+T_{T}^{s}}=\sqrt{1-D_{T}^{2}}$,

$\mathrm{c}_{T}=\cos \Delta_{T}, \mathrm{~s}_{T}=\sin \Delta_{T}, \Delta_{T}=\varphi_{T}^{p}-\varphi_{T}^{s}$

Please note that this definition differs in two ways from the definition in Chipman (2009b): the retardance is defined differently there $\left(\Delta_{X}=\varphi_{X}{ }^{S}-\varphi_{X}{ }^{P}\right)$, and we denote with $D$ the horizontal diattenuation parameter $d_{h}$ (Chipman 2009b) and not the diattenuation magnitude $D_{\text {mag }}=|D|$ (see Supplement Sect. S4). The Müller matrix for the reflecting part of the PBS Eq. (16) includes a mirror reflection (Supplement Sect. S6) with the corresponding intensity reflection coefficients (reflectance) for light polarised parallel $\left(R_{\mathrm{p}}=T_{R}{ }^{\mathrm{p}}\right)$ and perpendicular $\left(R_{\mathrm{S}}=T_{R}{ }^{\mathrm{s}}\right)$ to the plane of incidence (Supplement Sect. S1) of the polarising beam splitter.

$\mathbf{M}_{R}=T_{R}\left(\begin{array}{cccc}1 & D_{R} & 0 & 0 \\ D_{R} & 1 & 0 & 0 \\ 0 & 0 & -Z_{R} \mathrm{c}_{R} & -Z_{R} \mathrm{~s}_{R} \\ 0 & 0 & Z_{R} \mathrm{~s}_{R} & -Z_{R} \mathrm{c}_{R}\end{array}\right)=T_{R}\left(\begin{array}{cccc}1 & 0 & 0 & 0 \\ 0 & 1 & 0 & 0 \\ 0 & 0 & -1 & 0 \\ 0 & 0 & 0 & -1\end{array}\right)\left(\begin{array}{cccc}1 & D_{R} & 0 & 0 \\ D_{R} & 1 & 0 & 0 \\ 0 & 0 & Z_{R} \mathrm{c}_{R} & Z_{R} \mathrm{~s}_{R} \\ 0 & 0 & -Z_{R} \mathrm{~s}_{R} & Z_{R} \mathrm{c}_{R}\end{array}\right)$

$T_{R}=\frac{T_{R}^{p}+T_{R}^{s}}{2}, D_{R}=\frac{T_{R}^{p}-T_{R}^{s}}{T_{R}^{p}+T_{R}^{s}}, Z_{R}=\frac{2 \sqrt{T_{R}^{p} T_{R}^{s}}}{T_{R}^{p}+T_{R}^{s}}=\sqrt{1-D_{R}^{2}}$,

$\mathrm{c}_{R}=\cos \Delta_{R}, \mathrm{~s}_{R}=\sin \Delta_{R}, \Delta_{R}=\varphi_{R}^{p}-\varphi_{R}^{s}$

In order to simplify the derivation of the equations, we describe both the reflecting and transmitting matrices with the matrix $\mathbf{M}_{S}$, and replace the subscript ${ }_{S}$ (for splitter) with ${ }_{T}$ (transmitting) or ${ }_{R}$ (reflecting) where appropriate, which means

$D_{S} \in\left\{D_{R}, D_{T}\right\}, \mathbf{M}_{S} \in\left\{\mathbf{M}_{R}, \mathbf{M}_{T}\right\}, I_{S} \in\left\{I_{R}, I_{T}\right\}$

It has to be emphasised that for this reason we cannot use the diattenuation magnitude $D_{m a g}$, which is always positive and almost exclusively used in other publications, but we have to use the diattenuation parameter $D$, which changes the sign when $T_{R}^{\mathrm{s}}$ becomes larger than $T_{R}^{\mathrm{p}}$ (see Supplement Sect. S3). Please keep also in mind that usually $D_{\mathrm{R}}<0$ that $\mathbf{M}_{R}$ includes an additional mirror reflection, and that fluxes measured after the PBS are not influenced by the addition of an ideal mirror reflection in the optical path. 


\subsection{Calibration, linear depolarisation ratio, and total signal}

Equation (6) shows the Stokes vectors of the transmitted $\left(\boldsymbol{I}_{T}\right)$ and reflected $\left(\boldsymbol{I}_{R}\right)$ channels, alias $\boldsymbol{I}_{S}$, after the polarising beam splitter $\mathbf{M}_{S}$ (PBS) without calibrator, i.e. $\mathbf{C}=\mathbf{1}=$ identity matrix. Equation (6) represents the standard measurement at the axial rotation of $0^{\circ}$, neglecting for now additional optics in $\mathbf{M}_{O}$.

$$
\begin{aligned}
& \boldsymbol{I}_{S}\left(0^{\circ}\right)=\eta_{S} \mathbf{M}_{S} \mathbf{F} \boldsymbol{I}_{L}=\eta_{S} \mathbf{M}_{S} \boldsymbol{I}_{\text {in }}= \\
& =\eta_{S} T_{S}\left(\begin{array}{cccc}
1 & D_{S} & 0 & 0 \\
D_{S} & 1 & 0 & 0 \\
0 & 0 & Z_{S} \mathrm{c}_{S} & Z_{S} \mathrm{~s}_{S} \\
0 & 0 & -Z_{S} \mathrm{~s}_{S} & Z_{S} \mathrm{c}_{S}
\end{array}\right) F_{11} I_{L}\left(\begin{array}{l}
1 \\
a \\
0 \\
0
\end{array}\right)=\eta_{S} T_{S} F_{11} I_{L}\left(\begin{array}{c}
1+D_{S} a \\
D_{S}+a \\
0 \\
0
\end{array}\right)
\end{aligned}
$$

The measured signals $I_{S}$ are

$$
I_{S}\left(0^{\circ}\right)=\eta_{S} T_{S} F_{11} I_{L}\left(1+D_{S} a\right)
$$

which correspond to the transmitted and reflected intensities, include the individual channels gains $\eta_{S}$, i.e. $\eta_{T}$ and $\eta_{R}$, which are the product of the electronic amplification of the detectors, the amplifiers, and of the optical attenuation due to polarisation insensitive attenuation of all optics including neutral density and interference filters. The latter is in general different in the two channels. We can solve the equation of the ratio of the measured reflected to the transmitted signals

$$
\frac{I_{R}}{I_{T}}\left(0^{\circ}\right)=\frac{\eta_{R} T_{R}\left(1+D_{R} a\right)}{\eta_{T} T_{T}\left(1+D_{T} a\right)}=\frac{\eta_{R}}{\eta_{T}} \frac{\left(T_{R}^{p}+T_{R}^{s} \delta\right)}{\left(T_{T}^{p}+T_{T}^{s} \delta\right)}
$$

for the linear depolarisation ratio $\delta$ if we know the calibration factor

$$
\eta \equiv \frac{\eta_{R} T_{R}}{\eta_{T} T_{T}}
$$

(with reflectance $T_{R}$ and transmittance $T_{T}$ for unpolarised light) and the transmission parameters of the polarising beam splitter $T_{T}^{\mathrm{p}}, T_{T}^{\mathrm{s}}, T_{R}{ }^{\mathrm{p}}$, and $T_{R}{ }^{\mathrm{s}}$ for the correction of its crosstalk. We could get the calibration factor $\eta$ already with the measurements in Eq. (21) if the light incident on the analyser were unpolarised, i.e. $a=0$. Otherwise, $\eta$ can be determined by means of calibration measurements, e.g. by rotating the PBS including the detectors by $+45^{\circ}$ or $-45^{\circ}$ about the optical axis (Eq. 23). 


$$
\begin{aligned}
& \boldsymbol{I}_{S}\left( \pm 45^{\circ}\right)=\eta_{S} \mathbf{M}_{S} \mathbf{R}\left( \pm 45^{\circ}\right) \mathbf{F} \boldsymbol{I}_{\text {in }}= \\
& =\eta_{S} \mathbf{M}_{S}\left(\begin{array}{cccc}
1 & 0 & 0 & 0 \\
0 & 0 & \mp 1 & 0 \\
0 & \pm 1 & 0 & 0 \\
0 & 0 & 0 & 1
\end{array}\right) F_{11} I_{L}\left(\begin{array}{l}
1 \\
a \\
0 \\
0
\end{array}\right)=\eta_{S} T_{S}\left(\begin{array}{cccc}
1 & D_{S} & 0 & 0 \\
D_{S} & 1 & 0 & 0 \\
0 & 0 & Z_{S} c_{S} & Z_{S} s_{S} \\
0 & 0 & -Z_{S} s_{S} & Z_{S} c_{S}
\end{array}\right) F_{11} I_{L}\left(\begin{array}{c}
1 \\
0 \\
\pm a \\
0
\end{array}\right)= \\
& =\eta_{S} T_{S} F_{11} I_{L}\left(\begin{array}{c}
1 \\
D_{S} \\
\pm a Z_{S} c_{S} \\
\mp a Z_{S} s_{S}
\end{array}\right)
\end{aligned}
$$

With the rotations $\mathbf{R}\left( \pm 45^{\circ}\right)$ it is intended to produce at the entrance of the PBS equal light intensities in the transmitted and reflected paths, independent of the atmospheric depolarisation. The error from an inaccurate $\pm 45^{\circ}$ alignment can be reduced by the $\Delta 90$ calibration explained in Sect. 5. From Eq. (23) we get the signal intensities

$I_{S}\left( \pm 45^{\circ}\right)=\eta_{S} T_{S} F_{11} I_{L}$

and the calibration factor $\eta$ from the signal ratio

$$
\frac{I_{R}}{I_{T}}\left( \pm 45^{\circ}\right)=\frac{\eta_{R} T_{R}}{\eta_{T} T_{T}}=\eta
$$

With known $\eta$ we can express the measured signal ratio $\delta^{*}$ in Eq. (21) as

$\delta^{*} \equiv \frac{1}{\eta} \frac{I_{R}}{I_{T}}\left(0^{\circ}\right)=\frac{I_{T}}{I_{R}}\left( \pm 45^{\circ}\right) \frac{I_{R}}{I_{T}}\left(0^{\circ}\right)=\frac{T_{T}}{T_{R}} \frac{T_{R}^{p}+T_{R}^{s} \delta}{T_{T}^{p}+T_{T}^{s} \delta}$

which is almost equal to the linear depolarisation ratio $\delta$ but still includes the diattenuation and crosstalk of the imperfect polarising beam splitter. From $\delta^{*}$ we retrieve the linear depolarisation ratio $\delta$

$\delta=\frac{\delta^{*} T_{R} T_{T}^{p}-T_{T} T_{R}^{p}}{T_{T} T_{R}^{s}-\delta^{*} T_{R} T_{T}^{s}}$

With the assumption of good PBSs

$T_{T}^{s} \ll 1 \Rightarrow\left\{T_{R}^{s} \approx 1, \quad T_{T} \approx 0.5 T_{T}^{p}, T_{R} \approx 0.5\left(1+T_{R}^{p}\right)\right\}$

we get an approximation

$\delta \approx \delta^{*}-T_{R}^{p}\left(1-\delta^{*}\right)$ 
Next we will determine the total lidar backscatter signal from the two signals $I_{T}$ and $I_{R}$ measured at $0^{\circ}$. This is the rangedependent signal, which we use for the inversion of the backscatter coefficient $F_{11}$ with the lidar inversion methods. From Eq. (20) we can get $F_{11}$ either from the transmitted or from the reflected signal

$$
F_{11}=\frac{I_{S}\left(0^{\circ}\right)}{\eta_{S} T_{S} I_{L}\left(1+D_{S} a\right)}
$$

The polarisation parameter $a$ can be extracted from the signal ratio in Eq. (21)

$$
a=\frac{\eta I_{T}-I_{R}}{I_{R} D_{T}-\eta I_{T} D_{R}}
$$

and substituted in Eq. (30) to yield

$$
I_{L} F_{11}=\frac{\eta_{T} T_{T} D_{T} I_{R}-\eta_{R} T_{R} D_{R} I_{T}}{\eta_{T} T_{T} \eta_{R} T_{R}\left(D_{T}-D_{R}\right)}=\frac{1}{D_{T}-D_{R}}\left(\frac{D_{T} I_{R}}{\eta_{R} T_{R}}-\frac{D_{R} I_{T}}{\eta_{T} T_{T}}\right)
$$

Equation (32) shows that we cannot determine an absolute $F_{l l}$ without an absolute calibration of the individual channel gains $\eta_{R}$ and $\eta_{T}$ and knowledge of the laser intensity $I_{L}$. However, for the lidar signal inversions, which use a reference value at a certain range or similar, we only need a relative, range-dependent $F_{11}$. Hence we can choose any of the rangeindependent parameters in Eq. (32), in which only $I_{T}$ and $I_{R}$ are range-dependent, which we cancel out and get

$$
F_{11} \propto D_{T} I_{R}-\eta D_{R} I_{T}=\frac{T_{T}^{p}-T_{T}^{s}}{T_{T}^{p}+T_{T}^{s}} I_{R}-\eta \frac{T_{R}^{p}-T_{R}^{s}}{T_{R}^{p}+T_{R}^{s}} I_{T} .
$$

In the case that the polarising beam splitter is ideal, i.e. $T_{T}^{\mathrm{p}}=T_{R}^{\mathrm{s}}=1$ and $T_{T}^{\mathrm{s}}=T_{R}^{\mathrm{p}}=0$, and hence $D_{R}=-1$ and $D_{T}=+1$, Eq. (33) becomes as expected

$$
F_{11} \propto I_{R}+\eta I_{T}
$$

Please bear in mind that in general $T_{R}{ }^{\mathrm{s}}>T_{R}{ }^{\mathrm{p}}$, and therefore $\left(T_{R}^{\mathrm{p}}-T_{R}^{\mathrm{s}}\right)<0$ and $D_{R}<0$ according to our definition in Eq. (17).

Summarising, we have to find the calibration factor $\eta$ and correct the crosstalk. $\delta$ is retrieved from two signals at $0^{\circ}$ represented by $\delta^{*}$, Eq. (26), plus two signals for the calibration factor at $\pm 45^{\circ}$, Eq. (25), and the knowledge of the PBS parameters $T_{T}^{\mathrm{p}}, T_{T}^{\mathrm{s}}, T_{R}^{\mathrm{p}}$, and $T_{R}^{\mathrm{s}}$ for the correction of the crosstalk.

\section{Complete Müller-Stokes lidar setup with rotation of optical elements}

In the previous section, a basic lidar setup is described with the Müller-Stokes formalism as an introduction, which includes only a horizontally linearly polarised laser, the matrices for the atmospheric aerosol backscattering and depolarisation, and the polarising beam splitter. In order to expand this setup to a realistic but still manageable model 
for a large variety of lidar systems and calibration techniques, we introduce in this section some concepts and parameters, which will enable us to describe the variety of setups with as few as possible equations.

The Stokes-Müller formalism (Chipman, 2009b) represents four linear equations (Eq. 35), which relate the four outputs with the four input Stokes parameters.

$$
\begin{aligned}
& \boldsymbol{I}_{\text {out }}=\left(\begin{array}{c}
I_{\text {out }} \\
Q_{\text {out }} \\
U_{\text {out }} \\
V_{\text {out }}
\end{array}\right)=\mathbf{M I}_{\text {in }}=\left(\begin{array}{llll}
M_{11} & M_{12} & M_{13} & M_{14} \\
M_{21} & M_{22} & M_{23} & M_{24} \\
M_{31} & M_{32} & M_{33} & M_{34} \\
M_{41} & M_{42} & M_{43} & M_{44}
\end{array}\right)\left(\begin{array}{l}
I_{\text {in }} \\
Q_{\text {in }} \\
U_{\text {in }} \\
V_{\text {in }}
\end{array}\right)= \\
& =M_{11} I_{\text {in }}\left(\begin{array}{cccc}
1 & m_{12} & m_{13} & m_{14} \\
m_{21} & m_{22} & m_{23} & m_{24} \\
m_{31} & m_{32} & m_{33} & m_{34} \\
m_{41} & m_{42} & m_{43} & m_{44}
\end{array}\right)\left(\begin{array}{l}
i_{\text {in }} \\
q_{\text {in }} \\
u_{\text {in }} \\
v_{\text {in }}
\end{array}\right)
\end{aligned}
$$

The lower-case matrix $\left(m_{i j}\right)$ and vector components on the right of Eq. (35) are normalised by their first element, i.e. the unpolarised transmission $M_{11}$ and the total intensity $I_{i n}$; hence $m_{l 1}=i_{i n}=1$. In the following we usually keep the variable $i_{\text {in }}$ in order to allow for later expansions of the equations. While the first Stokes vector parameter $I_{\text {out }}$ can be directly detected with a photon detector, the other output Stokes parameters can each be determined with two measurements of output intensities using additional polarisation elements (Chipman, 2009a) (see Eq. S.2.2 in the Supplement). We derive the backscatter coefficient $F_{l l}$ and the linear polarisation parameter $a$ of the Müller matrix $\mathbf{F}$ of the atmosphere (see Sect. 2.1) from the first two equations of $I_{\text {out }}$ and $Q_{\text {out }}$ in Eq. (35), which in turn are determined from the two measurements of $I_{R}$ and $I_{T}$ using the two orthogonal linear analysers of the polarising beam splitter. For the determination of each additional unknown parameter we need additional measurements. For the relative calibration factor $\eta$ of the two polarisation signals $I_{R}$ and $I_{T}$ we use an additional calibrator element with Müller matrix $\mathbf{C}$. The lidar setup shown in Fig (1) is described by Eq. (6), i.e. $\boldsymbol{I}_{S}=\eta_{S} \mathbf{M}_{S} \mathbf{C} \mathbf{M}_{O} \mathbf{F} \mathbf{M}_{E} \boldsymbol{I}_{L}$, where the matrices $\mathbf{M}_{T, R}$ (alias $\mathbf{M}_{S}$ ) represent the two paths of the polarising beam splitter, i.e. subscripts $T$ for transmission and $R$ for reflection. Since the laser in our model can be arbitrarily polarised and because "parallel" and "perpendicular" are defined relative to the incident plane of a beam splitter (superscripts $p$ and $s$, respectively; see Supplement Sect. S1) and do not necessarily describe the polarisation behind it with respect to the laser polarisation, we cannot use these terms here for the two branches behind the polarising beam splitter. $\mathbf{C}(\Psi)$ describes the calibrator matrix, which can be a mechanical rotation of the detection optics by $\Psi$ or an optical device as a polarising sheet filter rotated by angle $\Psi$, for example. The purpose of the calibrator device is to produce equal intensities for both polarisation channels, independent of the laser light polarisation and independent of backscattering characteristics of the atmosphere. This is achieved, for example, with an ideal polarising sheet filter oriented at $45^{\circ}$ with respect to the incident plane of the PBS. The calibration factor $\eta$ of the relative sensitivity of both polarisation channels can be retrieved from the ratio of the measured intensities. The calibration factor includes electronic gains and the polarisation transmission of optical elements behind the calibrator. In our model 
the calibrator can be at three different positions in the optical chain, which are indicated by the red blocks in Fig. 2 . The calibrator positions and the respective equations are the following:

behind the laser emitter optics $\mathbf{M}_{E}$

before the telescope / receiver optics $\mathbf{M}_{O}$

before the polarising beam splitter $\mathbf{M}_{S}$

$$
\boldsymbol{I}_{S}=\eta_{S} \mathbf{M}_{S} \mathbf{M}_{O} \mathbf{F C} \mathbf{M}_{E} \boldsymbol{I}_{L}=\eta_{S} \mathbf{M}_{S} \mathbf{M}_{o} \mathbf{F C} \boldsymbol{I}_{i n}
$$

$$
\boldsymbol{I}_{S}=\eta_{S} \mathbf{M}_{S} \mathbf{M}_{O} \mathbf{C} \mathbf{F} \mathbf{M}_{E} \boldsymbol{I}_{L}=\eta_{S} \mathbf{M}_{S} \mathbf{M}_{O} \mathbf{C} \boldsymbol{I}_{i n}
$$$$
\boldsymbol{I}_{S}=\eta_{S} \mathbf{M}_{S} \mathbf{C} \mathbf{M}_{O} \mathbf{F} \mathbf{M}_{E} \boldsymbol{I}_{L}=\eta_{S} \mathbf{M}_{S} \mathbf{C I}
$$

In the case that the telescope and/or the collimating lens do not change the state of polarisation of the incoming light, the placement of the calibrator after those elements is equivalent to the position before the telescope.

We develop the equations for all three positions of the calibrator, and additionally for the calibration with an unpolarised light source before the receiving optics (Sect. 6). In the equations we use as calibrator elements the Müller matrix $\mathbf{C}$ as a place holder for any sort of calibrator, which are $\mathbf{M}_{r o t}$ for mechanical rotation or by means of a $\lambda / 2$ plate, $\mathbf{M}_{P}$ for a linear polariser, $\mathbf{M}_{Q W}$ for a $\lambda / 4$ plate, and $\mathbf{M}_{C P}$ for a circular polariser.

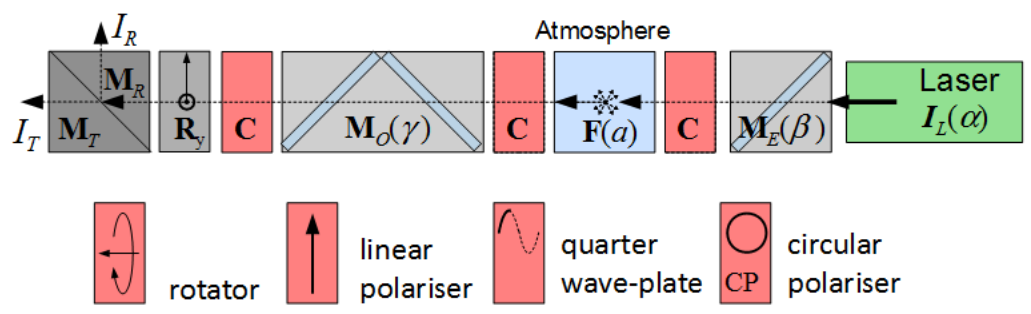

Figure 2. Schematic of a two-channel, polarisation-sensitive lidar setup (compare Fig. 1) with Müller matrix block elements and different calibrator (red block) positions (top), and three options for the calibrator $\mathbf{C}$ (bottom). $\boldsymbol{I}_{L}$ : laser Stokes vector, $\mathbf{M}_{E}$ : emitter optics; F: atmospheric backscatter matrix with polarisation parameter a; $\mathbf{M}_{O}$ : receiver optics; $\mathbf{R}_{\mathrm{y}}$ : rotation matrix for the $0^{\circ}(\mathrm{y}=+1)$ and $90^{\circ}(\mathrm{y}=-1)$ detection setup (see text); $\mathbf{M}_{T, R}$ : transmitted and reflected part of the polarising beam splitter; $\boldsymbol{I}_{T, R}$ : transmitted and reflected detection signals. Angles $\alpha, \beta$, and $\gamma$ are rotations around the optical axis.

\subsection{The analyser $<$ bra $\mid$ and input $\mid$ ket $>$ vectors}

The general structure of all the considered lidar setups can be described with three groups of optical elements: elements before the calibrator, the calibrator, and elements behind the calibrator. To simplify the equations, we combine the

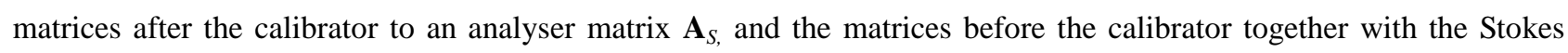
vector of the laser beam $\boldsymbol{I}_{L}$ to an input Stokes vector $\boldsymbol{I}_{i n}$. Since $\mathbf{A}_{S}$ and $\boldsymbol{I}_{i n}$ are the same for all calibrator types, they have to be derived only once and can then be used for the different setups. "After" and "before" denote the order with respect to the light direction, i.e. from right to left in the Müller-Stokes equations.

Since photodetectors are, in general, insensitive to the polarisation, we measure the intensity $I_{S}$ at the detector, which is the first parameter of the output Stokes vector. $I_{S}$ is determined by the top row of a matrix $\mathbf{A}_{S}$ and an input vector $\boldsymbol{I}_{i n}$. 


$$
\left(\begin{array}{c}
I_{S} \\
- \\
- \\
-
\end{array}\right)=\eta_{S} \mathbf{A}_{S} \boldsymbol{I}_{i n}=\eta_{S}\left(\begin{array}{cccc}
A_{11} & A_{12} & A_{13} & A_{14} \\
- & - & - & - \\
- & - & - & - \\
- & - & - & -
\end{array}\right)\left(\begin{array}{c}
I_{i n} \\
Q_{i n} \\
U_{i n} \\
V_{i n}
\end{array}\right)=\eta_{S}\left(\begin{array}{c}
A_{11} I_{i n}+A_{12} Q_{i n}+A_{13} U_{i n}+A_{14} V_{i n} \\
- \\
- \\
-
\end{array}\right)
$$

Using the <bra|ket> matrix-vector notation (see 0 and 0 ), we define for this work the row vector $<\mathbf{A}_{S} \mid$ as the top row of a matrix $\mathbf{A}_{S}$

$$
\left\langle\mathbf{A}_{S}\right|=\left\langle\begin{array}{llll}
A_{11} & A_{12} & A_{13} & A_{14}
\end{array}\right|
$$

and use analogously the column vector $\left|\boldsymbol{I}_{i n}\right\rangle$. With this notation the equation for the intensity $I_{S}$ can be written as

$$
\begin{aligned}
& I_{S}=\eta_{S}\left\langle\mathbf{A}_{S} \mid \boldsymbol{I}_{\text {in }}\right\rangle=\eta_{S} I_{\text {in }}\left\langle A_{11} \quad A_{12} \quad A_{13} \quad A_{14} \mid I_{\text {in }} \quad Q_{\text {in }} \quad U_{\text {in }} \quad V_{\text {in }}\right\rangle= \\
& =\eta_{S} I_{\text {in }}\left(A_{11} I_{\text {in }}+A_{12} Q_{\text {in }}+A_{13} U_{\text {in }}+A_{14} V_{\text {in }}\right)
\end{aligned}
$$

For example, the equation for signal $I_{S}$ of a calibration measurement with the calibrator before the PBS (see Eq. 38) can be expressed as

$$
I_{S}(\mathrm{y}, \mathrm{x}, \varepsilon)=\eta_{S}\left\langle\mathbf{M}_{S} \mathbf{R}_{\mathrm{y}}\left|\mathbf{C}\left(\mathrm{x} 45^{\circ}+\varepsilon\right)\right| \mathbf{M}_{O} \mathbf{F} \mathbf{M}_{E} \boldsymbol{I}_{L}\right\rangle=\eta_{S}\left\langle\mathbf{A}_{S}|\mathbf{C}| \boldsymbol{I}_{\text {in }}\right\rangle
$$

and the respective standard measurement signals without the calibrator can be expressed with the same vectors $\left\langle\mathbf{A}_{S}\right|$ and $\boldsymbol{I}_{\text {in }}>$ as

$$
I_{S}(\mathrm{y})=\eta_{S}\left\langle\mathbf{M}_{S} \mathbf{R}_{\mathrm{y}} \mid \mathbf{M}_{O} \mathbf{F} \mathbf{M}_{E} \boldsymbol{I}_{L}\right\rangle=\eta_{S}\left\langle\mathbf{A}_{S} \mid \boldsymbol{I}_{\text {in }}\right\rangle
$$

This split-up of the equations in an analyser bra vector and an input Stokes ket vector is similar to the split-up in instrumental vectors of the transmitter and receiver in Kaul et al. (2004) and Volkov et al. (2015).

In Eqs. (42) and (43) we already used the binary operators $y$, x, the parameter $\varepsilon$ for different rotation angles, and the rotation matrix $\mathbf{R}_{\mathrm{y}}$, which will be explained in detail in Sect. 3.3.

\subsection{Laser polarisation and atmospheric depolarisation}

The light leaving commercial Nd:YAG lasers is usually linearly polarised. Manufacturers often specify a polarisation "purity" > $95 \%$ or similar, which is not very accurate. Actually, the laser light is often much better polarised, but the measurement of the polarisation of individual lasers in a series is expensive and it can change during the operation and with ageing of the laser. Probably for that reason the manufacturers seem to specify a lower limit which they can assure under all circumstances. A secure method to ensure a high degree of linear polarisation is to use a polariser as the last element at the laser output. Often the orientation of the laser polarisation relative to the orientation of the polarising beam splitter in the receiving optics is not well known, firstly because the state of polarisation of short laser pulses with high power is difficult to measure accurately, secondly because the state of polarisation of the laser can change during 
the operation of the laser over periods with changing environmental conditions. Hence we consider a possible rotation $\alpha$ of the plane of horizontal linear polarisation of the laser (laser rotation). Furthermore, beam expanders and especially steering mirrors after the laser can degrade the degree of linear polarisation considerably producing elliptical polarised light. Hence we start with an emitter Stokes vector with arbitrary state of polarisation leaving the laser, which includes all effects of cleaning, shaping, and steering optics

$$
\boldsymbol{I}_{E}=\mathbf{M}_{E} \boldsymbol{I}_{L}=T_{E} I_{L}\left|i_{E} \quad q_{E} \quad u_{E} \quad v_{E}\right\rangle
$$

We will develop all equations first for a general emitter beam polarisation as in Eq. (44), and then as an explicit example for a linearly polarised laser with intensity $I_{L}$ and laser rotation $\alpha$ (see 0 ) to elaborate the errors due to misalignments of the calibration and measurement optics.

$$
\boldsymbol{I}_{L}(\alpha)=I_{L}\left|1 \quad \mathrm{c}_{2 \alpha} \quad \mathrm{s}_{2 \alpha} \quad 0\right\rangle
$$

Depolarisation of the laser (with linear polarisation parameter $a_{L}$ ), caused by volume or surface scattering in or on optical elements, is hardly probable, and the scattered radiation reaching the lidar telescope would be negligible. However, it is briefly covered in Supplement Sect. S3. The Stokes vector $\boldsymbol{I}_{F}$, which is reflected by the atmosphere with scattering matrix $\mathbf{F}(a)$ with linear polarisation parameter $a$ from a generally polarised emitter $\boldsymbol{I}_{E}$, is (see Supplement Sect. S3)

$$
\frac{\boldsymbol{I}_{F}(a)}{F_{11} T_{E} I_{L}}=\frac{\mathbf{F}(a)\left|\mathbf{M}_{E} \boldsymbol{I}_{L}\right\rangle}{F_{11} T_{E} I_{L}}=\left|i_{E} \quad a q_{E} \quad-a u_{E} \quad(1-2 a) v_{E}\right\rangle
$$

\subsection{Receiver optics and calibrator}

In order to investigate the effect of misalignments of the optical elements on the final measurement and the calibration results, i.e. the total signal and the linear depolarisation ratio, we apply to each optical element in Eqs. (36) to (38) an additional rotation error about the optical axis (see Fig. 2). The reference coordinate system is in general defined by the incident plane of the polarising beam splitter (Fig. 3); therefore no rotation error is considered in $\mathbf{M}_{S}$. Nevertheless, the polarising beam splitter can be mechanically rotated by $90^{\circ}$ in some existing lidar systems without changing the rest of the setup. We include this additional fixed rotation by introducing the rotation matrix $\mathbf{R}_{\mathrm{y}}$ with the polarising beam splitter orientation parameter $\mathrm{y}$ (Fig. 3). For $\mathrm{y}=+1$ the parallel laser polarisation is detected in the transmitted channel and for $y=-1$ in the reflected channel. This seems a bit confusing, but it is necessary to get control of all the actually existing lidar setups. The rotation matrix $\mathbf{R}_{\mathrm{y}}$ is shown in Eq. (47).

$$
\mathbf{R}_{\mathrm{y}}=\mathbf{R}(\mathrm{y})=\left(\begin{array}{llll}
1 & 0 & 0 & 0 \\
0 & \mathrm{y} & 0 & 0 \\
0 & 0 & \mathrm{y} & 0 \\
0 & 0 & 0 & 1
\end{array}\right) \Rightarrow \begin{gathered}
\mathbf{R}(\mathrm{y}=-1)=\mathbf{R}\left(90^{\circ}\right) \\
\mathbf{R}(\mathrm{y}=+1)=\mathbf{R}\left(0^{\circ}\right)
\end{gathered}
$$




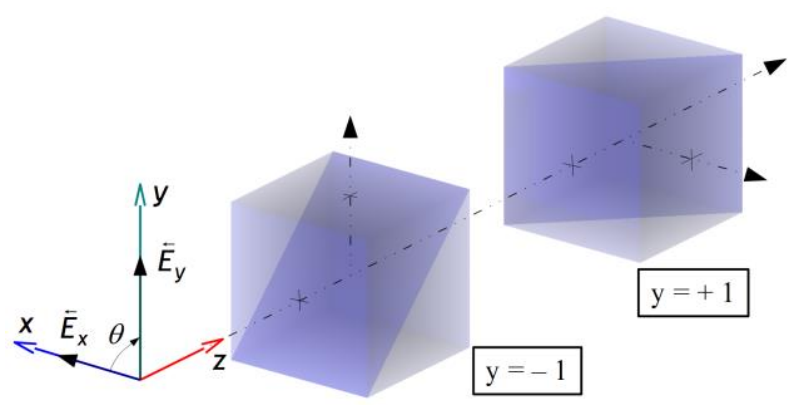

Figure 3. Definition of the global coordinate reference system and the binary operator $y$ with respect to the incident plane of the polarising beam splitter. If the polarising beam splitter orientation parameter $\mathrm{y}=+1$, the vibration of the horizontal linear polarisation with vector $\boldsymbol{E}_{x}$ is parallel to the plane of incidence, while for $\mathrm{y}=-1$ it is perpendicular.

The whole lidar system shown in Fig. 2 is then described by Eq. (48) with rotation angles $\alpha, \beta, \gamma$, and $\Psi$ around the optical axis.

$$
\boldsymbol{I}_{S}(\mathrm{y}, \Psi, \gamma, a, \beta, \alpha)=\eta_{S} \mathbf{M}_{S} \mathbf{R}(\mathrm{y}) \mathbf{C}(\Psi) \mathbf{M}_{O}(\gamma) \mathbf{F}(a) \mathbf{M}_{E}(\beta) \boldsymbol{I}_{L}(\alpha)
$$

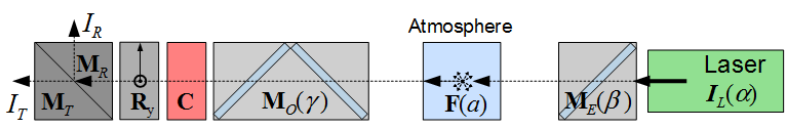

It would be possible to include the $\mathbf{R}_{\mathrm{y}}$ rotation by changing the laser angle $\alpha$ in Eq. (48), but we choose to do it before the polarising beam splitter for two reasons: first we want to use the angle $\alpha$ only for rotation errors, and ,second, in some lidar systems a rotation of the receiving optics is used for the calibration, and with these setups a change between the two $\mathbf{R}_{y}$ versions of a lidar is easily accomplished and can be used for certain test measurements without changing the rest of the equations. On the other hand, an arbitrary rotation of the laser polarisation is usually not possible. A rotation $\gamma$ of a retarding diattenuator $\mathbf{M}_{O}$ can complicate the equations considerably, as it converts linearly polarised light into elliptically polarised, which cannot be analysed by a simple polarising beam splitter. Therefore, diattenuating and retarding optics before the polarising beam splitter should be carefully oriented with their eigenaxes parallel to the ones of the polarising beam splitter to avoid the resulting uncertainties. Such an element can, for example, be a dichroic beam splitter, which does not reflect exactly to $0^{\circ}$ or $90^{\circ}$. For what we call $\Delta 90$ calibration, we use two calibrator orientations $\mathbf{C}(\Psi)$ with

$\Psi^{+}=+45^{\circ}+\varepsilon$
$\Psi^{-}=-45^{\circ}+\varepsilon$

so that

$\Psi^{+}-\Psi^{-}=90^{\circ}$ 
We choose these special angles because in the geometric mean of two calibrations at orientations exactly $90^{\circ}$ apart the error terms sometimes compensate very well. Note that the $\Delta 90$ error angle $\varepsilon$ describes the rotational misalignment of the whole $\Delta 90$ calibrator setup with respect to the polarising beam splitter, not the error in the $90^{\circ}$ difference. So, $\pm 45^{\circ}$ means either $+45^{\circ}$ or $-45^{\circ}$, and $\Delta 90$ means the combination of measurements at $+45^{\circ}+\varepsilon$ and $-45^{\circ}+\varepsilon$. To obtain general equations, we combine these angles using the binary operator $\mathrm{x}$ for calibrations

$\mathrm{x}= \pm 1: \Psi(\mathrm{x}, \varepsilon)=\mathrm{x} 45^{\circ}+\varepsilon$

We use this definition in a setup with a rotation calibrator $\mathbf{M}_{\text {rot }}($ Sect. 7)

$\mathbf{C}(\Psi, \mathrm{h})=\mathbf{M}_{r o t}\left(\mathrm{x} 45^{\circ}+\varepsilon, \mathrm{h}\right)=\mathbf{M}_{r o t}(\mathrm{x}, \varepsilon, \mathrm{h})=\mathbf{R}\left(\mathrm{x} 45^{\circ}\right) \mathbf{R}(\varepsilon) \mathbf{M}_{\mathrm{h}}$

with the binary operator $\mathrm{h}$ to discern between a mechanical $(\mathrm{h}=+1)$ and a $\lambda / 2$ plate rotation (Supplement $\mathrm{S} .10 .15)$ and can express the four equations for the reflected and transmitted signals $I_{R}$ and $I_{T}$ of the two calibration measurements at $\Psi= \pm 45^{\circ}+\varepsilon$ with Eq. (53)

$$
I_{S}(\mathrm{y}, \mathrm{x}, \varepsilon, \mathrm{h}, \gamma, a, \beta, \alpha)=\eta_{S}\left\langle\mathbf{M}_{S} \mathbf{R}_{\mathrm{y}}\left|\mathbf{M}_{r o t}(\mathrm{x}, \varepsilon, \mathrm{h})\right| \mathbf{M}_{o}(\gamma) \mathbf{F}(a) \mathbf{M}_{E}(\beta) \boldsymbol{I}_{L}(\alpha)\right\rangle
$$

and the four equations for the standard measurements at $\Psi=0^{\circ}(\mathrm{y}=+1)$ and $\Psi=90^{\circ}(\mathrm{y}=-1)$ using the same analyser and input Stokes vectors with just another formula Eq. (54)

$$
I_{S}(\mathrm{y}, \varepsilon, \mathrm{h}, \gamma, a, \beta, \alpha)=\eta_{S}\left\langle\mathbf{M}_{S} \mathbf{R}_{\mathrm{y}}\left|\mathbf{R}(\varepsilon) \mathbf{M}_{\mathrm{h}}\right| \mathbf{M}_{O}(\gamma) \mathbf{F}(a) \mathbf{M}_{E}(\beta) \boldsymbol{I}_{L}(\alpha)\right\rangle
$$

Using the rotation calibrator we have to consider the same alignment error $\varepsilon$ for the standard measurements at 0 and $90^{\circ}$ as for the calibration at the $\pm 45^{\circ}$ because this calibrator is not removed from the lidar setup after the calibration measurements. Please note that $\varepsilon=0$ for all other calibrators.

\section{Retrieval of the total signal and of the linear depolarisation ratio}

The final goal of this work is to investigate how the polarisation calibration factor, the linear depolarisation ratio, and the total lidar signal can be retrieved from the measurements $I_{T}$ and $I_{R}$, how much the various rotational misalignments and the crosstalk of the calibrator influence them, and how the deviations can possibly be corrected. The standard measurement signals $I_{S}$ in Eq. (54) include a rotational error $\varepsilon$ before the polarising beam splitter.

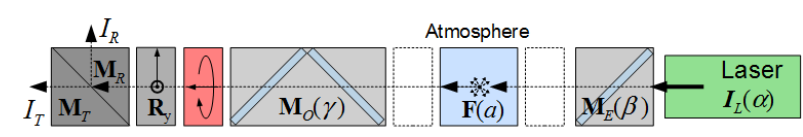

We get Eq. (55) for the analyser part with Eqs. (D5), (S.5.1.6), and (S.10.15.2), and with the most general input $\boldsymbol{I}_{E}$ from Eq. (E31) with atmospheric polarisation parameter $a$ we get the signal $I_{S}$ from Eq. (S.7.1.2)

$$
\left\langle\mathbf{A}_{S}(\mathrm{y})\right| \mathbf{R}(\varepsilon, \mathrm{h})=\left\langle\mathbf{M}_{S} \mathbf{R}_{\mathrm{y}}\right| \mathbf{R}(\varepsilon) \mathbf{M}_{\mathrm{h}}=T_{S}\left\langle 1 \quad \mathrm{yc}_{2 \varepsilon} D_{S} \quad-\mathrm{yhs}_{2 \varepsilon} D_{S} \quad 0\right|
$$




$$
\begin{aligned}
& \frac{I_{S}}{\eta_{S} T_{S} T_{r o t} T_{O} F_{11} T_{E} I_{L}}=\frac{\left\langle\mathbf{A}_{S}(\mathrm{y})\left|\mathbf{R}(\varepsilon) \mathbf{M}_{\mathrm{h}}\right| \boldsymbol{I}_{i n}(\gamma, a)\right\rangle}{T_{S} T_{r o t} T_{O} F_{11} T_{E} I_{L}}=\frac{\left\langle\mathbf{M}_{S} \mathbf{R} \mathrm{y}\left|\mathbf{R}(\varepsilon) \mathbf{M}_{\mathrm{h}}\right| \mathbf{M}_{O}(\gamma) \mathbf{F}(a) \boldsymbol{I}_{E}\right\rangle}{T_{S} T_{r o t} T_{O} F_{11} T_{E} I_{L}}= \\
& =\left(1+\mathrm{y} D_{S} D_{O} \mathrm{c}_{2 \gamma+\mathrm{h} 2 \varepsilon}\right) i_{E}-\mathrm{y}_{S} Z_{O} \mathrm{~s}_{O} \mathrm{~s}_{2 \gamma+\mathrm{h} 2 \varepsilon} v_{E}+ \\
& +a\left\{D_{O}\left(\mathrm{c}_{2 \gamma} q_{E}-\mathrm{s}_{2 \gamma} u_{E}\right)+\mathrm{y} D_{S}\left[\left(\mathrm{c}_{2 \varepsilon} q_{E}+\mathrm{s}_{\mathrm{h} 2 \varepsilon} u_{E}\right)-\mathrm{s}_{2 \gamma+\mathrm{h} 2 \varepsilon}\left(W_{O}\left(\mathrm{~s}_{2 \gamma} q_{E}+\mathrm{c}_{2 \gamma} u_{E}\right)-2 Z_{O} \mathrm{~s}_{O} v_{E}\right)\right]\right\}
\end{aligned}
$$

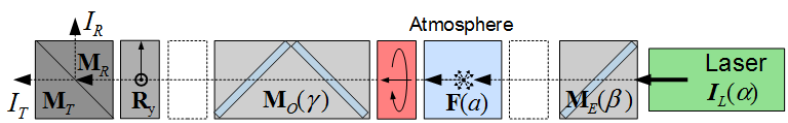

In the case that the rotational error is before the receiving optics, we get Eq. (57) from Eq. (S.7.2.1) with Eq. (D7) for the analyser part and (E26) for the input vector.

$$
\begin{aligned}
& \frac{I_{S}}{\eta_{S} T_{S} T_{O} T_{r o t} F_{11} T_{E} I_{L}}=\frac{\left\langle\mathbf{A}_{S}(\mathrm{y}, \gamma) \| \boldsymbol{I}_{i n, \varepsilon}(\varepsilon, \mathrm{h}, a)\right\rangle}{T_{S} T_{O} T_{r o t} F_{11} T_{E} I_{L}}=\frac{\left\langle\mathbf{M}_{S} \mathbf{R}_{\mathrm{y}} \mathbf{M}_{O}(\gamma) \| \mathbf{R}(\varepsilon) \mathbf{M}_{\mathrm{h}} \mathbf{F}(a) \boldsymbol{I}_{E}\right\rangle}{T_{S} T_{O} T_{r o t} F_{11} T_{E} I_{L}}= \\
& =\left(1+\mathrm{y} D_{O} D_{S} \mathrm{c}_{2 \gamma}\right) i_{E}-\mathrm{y} D_{S} Z_{O} \mathrm{~s}_{O} \mathrm{~s}_{2 \gamma} \mathrm{h} v_{E}+ \\
& +a\left\{\begin{array}{l}
D_{O}\left[\mathrm{c}_{2 \gamma-2 \varepsilon} q_{E}-\mathrm{s}_{2 \gamma-2 \varepsilon} \mathrm{h} u_{E}\right]+ \\
+\mathrm{y} D_{S}\left[\left(\mathrm{c}_{2 \varepsilon} q_{E}+\mathrm{s}_{2 \varepsilon} \mathrm{h} u_{E}\right)-\mathrm{s}_{2 \gamma}\left(W_{O}\left(\mathrm{~s}_{2 \gamma-2 \varepsilon} q_{E}+\mathrm{c}_{2 \gamma-2 \varepsilon} \mathrm{h} u_{E}\right)-2 Z_{O} \mathrm{~s}_{O} \mathrm{~h} v_{E}\right)\right]
\end{array}\right\}
\end{aligned}
$$

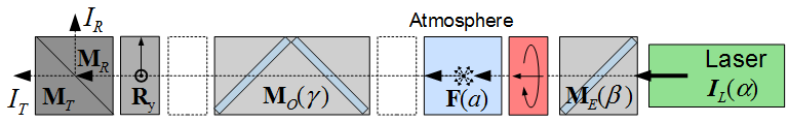

The case of rotational error behind the emitter optics can be retrieved from Eq. (57) by simply replacing $\varepsilon$ with $-\varepsilon$ (see Supplement Sect. S7.3). Special cases of $\boldsymbol{I}_{E}$ for Eqs. (56) and (57) can be found in Sect. E2.

\subsection{General formulations for the total signal and the linear depolarisation ratio}

From Eqs. (56) and (57) we see that all standard signals $I_{S}$ can be expressed by introducing two parameters $G_{S}$ and $H_{S}$ for the terms without and with atmospheric polarisation, respectively,

$$
I_{S}=\eta_{S} T_{S} T_{O} T_{r o t} F_{11} T_{E} I_{L}\left(G_{S}+a H_{S}\right)
$$

Using Eq. (56) as an example, the two parameters are

$$
\begin{aligned}
& G_{S}(\mathrm{y}, \varepsilon, \mathrm{h}, \gamma)=\left(1+\mathrm{y} D_{S} D_{O} \mathrm{c}_{2 \gamma+\mathrm{h} 2 \varepsilon}\right) i_{E}-\mathrm{y} D_{S} Z_{O} \mathrm{~s}_{O} \mathrm{~s}_{2 \gamma+\mathrm{h} 2 \varepsilon} v_{E} \\
& H_{S}(\mathrm{y}, \varepsilon, \mathrm{h}, \gamma, \beta, \alpha)= \\
& \quad=D_{O}\left(\mathrm{c}_{2 \gamma} q_{E}-\mathrm{s}_{2 \gamma} u_{E}\right)+\mathrm{y} D_{S}\left[\left(\mathrm{c}_{2 \varepsilon} q_{E}+\mathrm{s}_{\mathrm{h} 2 \varepsilon} u_{E}\right)-\mathrm{s}_{2 \gamma+\mathrm{h} 2 \varepsilon}\left(W_{O}\left(\mathrm{~s}_{2 \gamma} q_{E}+\mathrm{c}_{2 \gamma} u_{E}\right)-2 Z_{O} \mathrm{~s}_{O} v_{E}\right)\right]
\end{aligned}
$$

Table 1 shows how their expressions simplify if some uncertainties are neglected. 
With Eq. (58) the measured signal ratio becomes

$\delta^{*}=\frac{1}{\eta} \frac{I_{R}}{I_{T}}=\frac{G_{R}+a H_{R}}{G_{T}+a H_{T}}$

with the calibration factor $\eta=\left(\eta_{R} T_{R}\right) /\left(\eta_{T} T_{T}\right)$, which has to be determined with one of the methods in the following chapters. $G_{S}$ and $H_{S}$ describe the polarisation crosstalk terms of the lidar setup depending on the diattenuation parameters $D$ and the retardation (described by $\mathrm{s}_{O}$ and $\mathrm{c}_{O}$ ) of the individual optical elements, depending on the relative rotation of the elements and on the polarisation parameter of the atmosphere $a$. From Eq. (60) we retrieve the general equations for the polarisation parameter $a$ in Eq. (61) and for the linear depolarisation ratio $\delta$ in Eq. (62) (compare Eq. 12).

$a=\frac{\delta^{*} G_{T}-G_{R}}{H_{R}-\delta^{*} H_{T}}$

$\delta=\frac{1-a}{1+a}=\frac{\delta^{*}\left(G_{T}+H_{T}\right)-\left(G_{R}+H_{R}\right)}{\left(G_{R}-H_{R}\right)-\delta^{*}\left(G_{T}-H_{T}\right)}$

Remember that $\delta^{*}$ and hence $a$ and $\delta$ are range-dependent. For the retrieval of the total lidar signal, which is equivalent to $F_{1 l}$, we substitute Eq. (61) in Eq. (58) in the transmitted or the reflected version of $I_{S} \in\left\{I_{T}, I_{R}\right\}$ and replace $\delta *$ with Eq. (60). Using the transmitted signal $I_{T}$ from Eq. (58) we get Eq. (63), and after some restructuring (see Eqs. S.8.1 and S.8.2) we get the attenuated backscatter coefficient Eq. (64).

$$
\begin{aligned}
& \eta_{T} T_{T} T_{O} F_{11} T_{E} I_{L}=\frac{I_{T}}{G_{T}+a H_{T}} \\
& F_{11}=\frac{1}{T_{O} T_{E} I_{L}} \frac{H_{R} \frac{I_{T}}{\eta_{T} T_{T}}-H_{T} \frac{I_{R}}{\eta_{R} T_{R}}}{H_{R} G_{T}-H_{T} G_{R}}
\end{aligned}
$$

For the inversion of the lidar signal we only need the relative attenuated backscatter coefficient, for which we can get a much simpler formula by removing all factors in Eq. (64) which are not range-dependent (compare Eq. 32ff.), which yields Eq. (65):

$$
F_{11} \propto \eta H_{R} I_{T}-H_{T} I_{R}
$$

The individual calibration methods can add errors and uncertainties due to additional optics with unknown diattenuation and retardation and due to rotation errors. The possible uncertainties of the calibration factor $\eta$ can be assessed from the analytical expressions of the gain ratio $\eta^{*}$ (see Sect. 5). 
For systems without a polarising beam splitter, i.e. pure backscatter lidars with one channel for each wavelength, the total signal is $I_{T}$ from the transmitted signal, but with $D_{S}=D_{T}=0$, and without calibrator $(=>\mathrm{h}=1)$ and without calibrator rotation error angle $\varepsilon$. Hence, we get from both Eqs. (56) and (57) the transmitted signal with Eq. (66):

$D_{T}=0, T_{T}=1, \varepsilon=0, \mathrm{y}=1 \Rightarrow$

$I_{T}=\eta_{T} T_{T} T_{O} F_{11} T_{E} I_{L}\left[i_{E}+a D_{O}\left(\mathrm{c}_{2 \gamma} q_{E}-\mathrm{s}_{2 \gamma} u_{E}\right)\right]$

which shows that there is a distortion of the total signal due to the receiver optics diattenuation and depending on the atmospheric depolarisation, even if the laser beam behind the emitter optics is perfectly horizontally linearly polarised and without receiver optics rotation. i.e. Eq. (66) with

$\gamma=0, T_{E}=1, i_{E}=q_{E}=1, u_{E}=0 \Rightarrow$

$I_{T}=\eta_{T} T_{O} F_{11} I_{L}\left[1+a D_{O}\right]$

Table 1. Parameters $G_{S}$ and $H_{S}$ and their simplifications when neglecting some uncertainties for the case of Eq. (56), i.e. rotational error $\boldsymbol{\varepsilon}$ before the polarising beam-splitter, - with a general emitter Stokes vector $\boldsymbol{I}_{E}=T_{E} I_{L}\left|i_{E} \quad q_{E} \quad u_{E} \quad v_{E}\right\rangle$ (see Sect. E2).

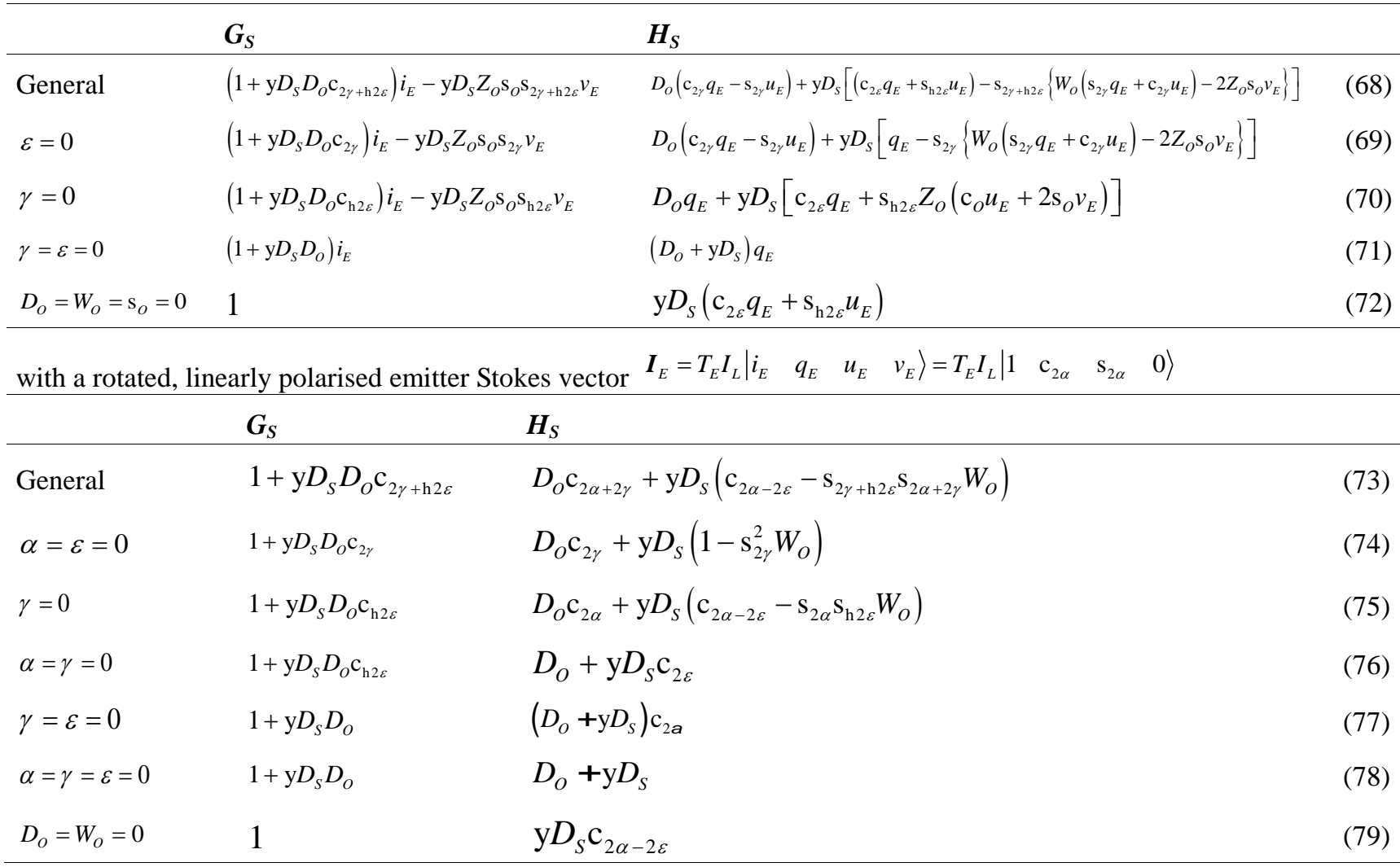




\section{$5 \quad$ The $45^{\circ}$ and $\Delta 90$ calibration, the gain ratios, and calibration factor}

The measured, apparent calibration factor $\eta^{*}$ of the polarisation channels, which we call in the following gain ratio in contrast to the calibration factor $\eta$, can be determined from the two calibration signals $I_{S}$, i.e. $I_{T}$ and $I_{R}$, with a calibrator at $+45^{\circ}$ or $-45^{\circ}$, which we call $45^{\circ}$ calibration (Eq. 80). The calibration factor $\eta$ is not directly measurable. Hence we need equations to retrieve $\eta$ from the measured $\eta^{*}$.

$\left.\begin{array}{l}\eta^{*}\left(+45^{\circ}\right)=\frac{I_{R}\left(+45^{\circ}\right)}{I_{T}\left(+45^{\circ}\right)} \\ \eta^{*}\left(-45^{\circ}\right)=\frac{I_{R}\left(-45^{\circ}\right)}{I_{T}\left(-45^{\circ}\right)}\end{array}\right\} \rightarrow \eta^{*}=\frac{I_{R}}{I_{T}}\left(\mathrm{x} 45^{\circ}\right)$

$\eta^{*}$ includes alignment errors and crosstalks. The theoretical dependence of these errors and crosstalks on the known parameters of our lidar model (Fig. 1) can be determined using the analytical expressions of Eqs. (81) and (82).

$$
\begin{aligned}
& I_{S}\left(\mathrm{y}, \mathrm{x} 45^{\circ}+\varepsilon\right)=\eta_{S}\left\langle\mathbf{A}_{S}(\mathrm{y})\left|\mathbf{C}\left(\mathrm{x} 45^{\circ}+\varepsilon\right)\right| \boldsymbol{I}_{\text {in }}\right\rangle \\
& \eta^{*}=\frac{I_{R}\left(\mathrm{y}, \mathrm{x} 45^{\circ}+\varepsilon\right)}{I_{T}\left(\mathrm{y}, \mathrm{x} 45^{\circ}+\varepsilon\right)}=\frac{\eta_{R}\left\langle\mathbf{A}_{R}(\mathrm{y})\left|\mathbf{C}\left(\mathrm{x} 45^{\circ}+\varepsilon\right)\right| \boldsymbol{I}_{\text {in }}\right\rangle}{\eta_{T}\left\langle\mathbf{A}_{T}(\mathrm{y})\left|\mathbf{C}\left(\mathrm{x} 45^{\circ}+\varepsilon\right)\right| \boldsymbol{I}_{\text {in }}\right\rangle}
\end{aligned}
$$

The theoretical correction $K$ of the gain ratio to get the calibrator factor can be retrieved from the analytical expression Eq. (83), which is then used to correct the measurement in Eqs. (84) or (85).

$$
\begin{aligned}
& K=\frac{\eta^{*}}{\eta}=\eta^{*} \frac{\eta_{T} T_{T}}{\eta_{R} T_{R}}=\frac{T_{T}}{T_{R}} \frac{\left\langle\mathbf{A}_{R}(\mathrm{y})\left|\mathbf{C}\left(\mathrm{x} 45^{\circ}+\varepsilon\right)\right| \boldsymbol{I}_{i n}\right\rangle}{\left\langle\mathbf{A}_{T}(\mathrm{y})\left|\mathbf{C}\left(\mathrm{x} 45^{\circ}+\varepsilon\right)\right| \boldsymbol{I}_{i n}\right\rangle} \\
& \eta=\frac{1}{K} \eta^{*}=\frac{1}{K} \frac{I_{R}}{I_{T}}\left(\mathrm{x} 45^{\circ}\right)
\end{aligned}
$$

Furthermore, additional equations for the estimation of the uncertainty of $\eta$ can be derived from Eq. (83). Since the errors due to $\varepsilon$ cancel out very well at orientations of the calibrator exactly $\Delta 90$ apart (i.e. $x= \pm 1$ ), as we will see in the following sections, a better estimation of the gain ratio can be retrieved from the geometric mean of the two gain ratios at $\pm 45^{\circ}$ (Eq. 85), which we call $\Delta 90$ calibration. The method of $90^{\circ}$ different polariser angles to reduce errors in polarimetric measurements seems to be common in ellipsometry (Nee, 2006).

$$
\eta_{\Delta 90}^{*} \equiv \sqrt{\eta^{*}\left(+45^{\circ}+\varepsilon\right) \eta^{*}\left(-45^{\circ}+\varepsilon\right)}=\sqrt{\frac{I_{R}\left(+45^{\circ}+\varepsilon\right)}{I_{T}\left(+45^{\circ}+\varepsilon\right)} \cdot \frac{I_{R}\left(-45^{\circ}+\varepsilon\right)}{I_{T}\left(-45^{\circ}+\varepsilon\right)}}
$$


While the two calibration signals $I_{T}$ and $I_{R}$ are taken at the same time, the two measurements for the $\Delta 90$ calibration at $\mathrm{x} 45^{\circ}+\varepsilon$ are done subsequently, and the atmosphere can change in between. If the gain ratio $\eta^{*}$ in Eq. (82) depends on the atmospheric polarisation parameter $a$, the $\Delta 90$ gain ratio $\eta^{*}{ }_{\Delta 90}$ in Eq. (85) depends also on the temporal change of $a$. In order to avoid this dependency, we either have to choose an appropriate setup and adjust it so that $\eta^{*}$ does not depend on $a$, or we have to choose a calibration range in which $a$ does not change with time. In the following we assume the latter, i.e. that the atmospheric polarisation parameter $a$ does not change in the calibration range between the two calibration measurements at $\times 45^{\circ}+\varepsilon$. This does not mean that the backscatter coefficient, an extrinsic parameter, must not change, but only that the aerosol composition with its intrinsic parameter $a$ remains the same and that the contribution of the air molecules to $a$ is negligible. Nevertheless, in Sect. 11 we describe a method to determine and consequently correct for $\varepsilon$, which is one of the major factors in the $a$ dependency of $\eta^{*}$. In Sects. 6 to 10 we derive $\mathbf{A}_{S}$ and $\boldsymbol{I}_{\text {in }}$ for several positions of the calibrator $\mathbf{C}$, and with that we will analyse special cases of the measurements $I_{S}$ and the retrieved calibration factor $\eta$. Figure 4 shows the steps in which the measurements are corrected for systematic errors by means of the model. If all system parameters of the model (Eqs. 56 and 57) are known, the crosstalk parameters $G_{S}$ and $H_{S}$ can be calculated (see Eqs. 68 to 79) and we only need to determine the calibration factor $\eta$ by means of calibration measurements in step 2 and its correction for crosstalk errors (step 3) as explained in Sects. 6 to 10 . Under certain conditions some instrumental parameters can be determined by means of additional calibration measurements (step 4) described under "special cases" in Sects. 6 to 10 and in Sects. 11 and 12.

\begin{tabular}{|c|c|c|}
\hline \multicolumn{2}{|l|}{ measurements } & model \\
\hline \multicolumn{3}{|l|}{ 1. standard measurements } \\
\hline \multicolumn{2}{|l|}{$I_{S}=\eta_{S}\left\langle\mathbf{A}_{S} \| \boldsymbol{I}_{\text {in }}\right\rangle \Rightarrow$} & $\begin{array}{ll}I_{S}=\eta_{S} T_{S} T_{O} T_{\text {rot }} F_{11} T_{E} I_{L}\left(G_{S}+a H_{S}\right) & \\
F_{11} \propto \eta H_{R} I_{T}-H_{T} I_{R} ; & \eta=\frac{\eta_{R} T_{R}}{\eta_{T} T_{T}} \\
\delta=\frac{\delta^{*}\left(G_{T}+H_{T}\right)-\left(G_{R}+H_{R}\right)}{\left(G_{R}-H_{R}\right)-\delta^{*}\left(G_{T}-H_{T}\right)} ; & \delta^{*}=\frac{1}{\eta} \frac{I_{R}}{I_{T}}\end{array}$ \\
\hline \multicolumn{3}{|l|}{ 2. calibration => gain ratio (Sect. 5 ) } \\
\hline \multicolumn{3}{|c|}{$I_{S}(\mathrm{x}, \varepsilon)=\eta_{S}\left\langle\mathbf{A}_{S}|\mathbf{C}(\mathrm{x}, \varepsilon)| \boldsymbol{I}_{\text {in }}\right\rangle \Rightarrow \eta_{\Delta 90}^{*}$} \\
\hline \multicolumn{3}{|c|}{ gain ratio correction $=>$ calibration factor (Sects. 6 to 10$)$} \\
\hline$\eta_{490}^{*}$ & & $\eta=\frac{\eta_{\Delta 90}^{*}}{K}$ \\
\hline \multicolumn{2}{|c|}{ 4. combined calibration measurements } & determination of instrumental parameters \\
\hline 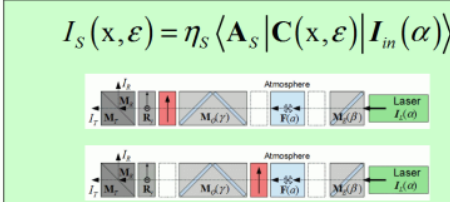 & $\Rightarrow$ & $\begin{array}{ll}D_{O} & (\text { Sects. 7.2, 8.2) } \\
v_{i n}, v_{E} & \text { (Sects. 9.1, 9.2, 9.3) } \\
\varepsilon & (\text { Sect. 11) } \\
\alpha & \text { (Sect. 12) }\end{array}$ \\
\hline
\end{tabular}

Figure 4. Four steps for calibrating and correcting the standard measurements for systematic errors by means of the model equations and additional calibration measurements. On the left side the measurements and their Müller matrix representations are listed and on the right side the scalar equations and their parameters, which can be retrieved from our model and from additional calibration measurements. 
The most general equation Eq. (86) for our lidar model, with e.g. a calibrator before the PBS, contains eight optical parameters of the four optical elements and the atmosphere, and four variables, i.e. the rotation angles of the optical elements and of the laser polarisation. Note that because detectors only detect the flux of light, the retardation of the polarising beam splitter $\Delta_{S}$ is irrelevant. For each setup we first derive the general formulations (Eq. 86). Then, in order to reduce the complexity of the equations and to carve out the most important and useful relations, we neglect certain parameters and variables in the detailed equations of special cases. We often omit the explicit description of the laser emitter optics $\mathbf{M}_{E}$ (Eq. 87), which means that we assume the light emitted to the atmosphere as arbitrarily polarised (see Sect. E2) $\boldsymbol{I}_{E}=\mathbf{M}_{E} \boldsymbol{I}_{L}=T_{E} I_{L}\left|i_{E} \quad q_{E} \quad u_{E} \quad v_{E}\right\rangle$. If necessary $\boldsymbol{I}_{E}$ can be expanded in the final equations by the appropriate ones in Appendix E. But we also consider the more simple case of a rotated linearly polarised laser $\boldsymbol{I}_{E}=\boldsymbol{I}_{L}=I_{L}\left|\begin{array}{llll}1 & \mathrm{c}_{2 \alpha} & \mathrm{s}_{2 \alpha} & 0\end{array}\right\rangle$. Furthermore, it is quite easy to remove the crosstalk of the polarising beam splitter $\mathbf{M}_{S}$ by means of additional polarisation filters behind it, which removes many terms in the Eq. (88). We call such an analyser "cleaned". The rotation $\gamma$ of the receiving optics $\mathbf{M}_{O}$ is very disturbing, which can be avoided in the very beginning of the lidar design (Eq. 89). And at last, this paper provides the tools to determine how good a calibrator must be to be considered ideal. With such a calibrator the equations become less complex (Eq. 90).

$$
\begin{array}{ll}
\boldsymbol{I}_{S}=\eta_{S} \mathbf{M}_{S}\left(D_{S}\right) \mathbf{R}_{\mathrm{y}} \mathbf{C}\left(D_{C}, \Delta_{C}, \varepsilon\right) \mathbf{M}_{O}\left(D_{O}, \Delta_{O}, \gamma\right) \mathbf{F}(a) \mathbf{M}_{E}\left(D_{E}, \Delta_{E}, \beta\right) \boldsymbol{I}_{L}(\alpha) \\
\boldsymbol{I}_{S}=\eta_{S} \mathbf{M}_{S}\left(D_{S}\right) \mathbf{R}_{\mathrm{y}} \mathbf{C}\left(D_{C}, \Delta_{C}, \varepsilon\right) \mathbf{M}_{O}\left(D_{O}, \Delta_{O}, \gamma\right) \mathbf{F}(a) & \boldsymbol{I}_{E} \\
\boldsymbol{I}_{S}=\eta_{S} \mathbf{M}_{S_{\text {clean }}} \mathbf{R}_{\mathrm{y}} \mathbf{C}\left(D_{C}, \Delta_{C}, \varepsilon\right) \mathbf{M}_{O}\left(D_{O}, \Delta_{O}, \gamma\right) \mathbf{F}(a) & \boldsymbol{I}_{E} \\
\boldsymbol{I}_{S}=\eta_{S} \mathbf{M}_{S}\left(D_{S}\right) \mathbf{R}_{\mathrm{y}} \mathbf{C}\left(D_{C}, \Delta_{C}, \varepsilon\right) \mathbf{M}_{O}\left(D_{O}, \Delta_{O}, 0\right) \mathbf{F}(a) & \boldsymbol{I}_{E} \\
\boldsymbol{I}_{S}=\eta_{S} \mathbf{M}_{S}\left(D_{S}\right) \mathbf{R}_{\mathrm{y}} \quad \mathbf{C}_{\text {ideal }} \quad \mathbf{M}_{O}\left(D_{O}, \Delta_{O}, \gamma\right) \mathbf{F}(a) & \boldsymbol{I}_{E}
\end{array}
$$

\section{Calibration with unpolarised input before the receiving optics}

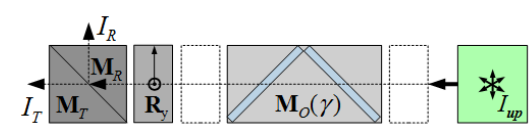

In principle, an additional light source with a known state of polarisation, which is placed before the telescope, can be used for the calibration. For other states of polarisation of the calibration light source the equations in Sect. (7.2) can be used together with the appropriate description of the input Stokes vector. But the beam from an additional light source has some disadvantages because it fills the apertures of the individual optical elements differently than the backscattered light from the lidar laser, and also the distribution of the incident angles on elements with limited acceptance angles, as dichroic beams splitters and interference filters, is different. Furthermore, the wavelength band of the light source is usually different from that of the lidar laser, which introduces wavelength-dependent transmission, diattenuation, and retardation effects. This can lead to errors in the calibration factor, which can additionally be range-dependent. Such 
errors are very difficult to assess. We therefore prefer to use the atmospheric backscatter of the lidar laser for the calibration, which provides the same spatial and angular characteristics and the same wavelengths for the calibration as for the measurements. Nevertheless, the output Stokes vector $\boldsymbol{I}_{S}$ of an unpolarised light source before the receiving optics is given by Eq. (91).

$$
\boldsymbol{I}_{S}=\eta_{S} \mathbf{M}_{S} \mathbf{R}_{\mathrm{y}} \mathbf{M}_{O} \boldsymbol{I}_{u p} \Rightarrow \mathbf{A}_{S}=\mathbf{M}_{S} \mathbf{R}_{\mathrm{y}} \mathbf{M}_{O} \text { and } \boldsymbol{I}_{i n}=\boldsymbol{I}_{u p}
$$

With the analyser vector from Eq. (D7) and the unpolarised input Stokes vector $\boldsymbol{I}_{\text {in }}$ before the lidar optics from Eq. (92) we get the calibration signals in Eq. (93).

$$
\begin{aligned}
& \boldsymbol{I}_{i n}=\boldsymbol{I}_{u p}=I_{u p} \mid \begin{array}{llll}
1 & 0 & 0 & 0
\end{array} \\
& I_{S}=\eta_{S}\left\langle\mathbf{M}_{S} \mathbf{R}_{\mathrm{y}} \mathbf{M}_{O}(\gamma)\right| \boldsymbol{I}_{u p}=\eta_{S} T_{S} T_{O} I_{u p}\left(1+\mathrm{y} D_{S} \mathbf{c}_{2 \gamma} D_{O}\right)
\end{aligned}
$$

The gain ratio can be retrieved directly with Eq. (93)

$$
\eta^{*}=\frac{I_{R}}{I_{T}}=\frac{\eta_{R} T_{R}}{\eta_{T} T_{T}} \frac{1+\mathrm{y} D_{R} D_{O} \mathrm{c}_{2 \gamma}}{1+\mathrm{y} D_{T} D_{O} \mathrm{c}_{2 \gamma}}=\eta \frac{1+\mathrm{y} D_{R} D_{O} \mathrm{c}_{2 \gamma}}{1+\mathrm{y} D_{T} D_{O} \mathrm{c}_{2 \gamma}}
$$

Error sources are the unknown receiver optics rotation $\gamma$ and the diattenuation $D_{O}$. With a cleaned analyser $\mathbf{M}_{S}$ (see Supplement Sect. S10.10) and $\gamma=0$ we get from Eq. (94)

$$
\frac{\eta^{*}}{\eta}=\frac{1-\mathrm{y} D_{O}}{1+\mathrm{y} D_{O}}
$$

With $D_{O}=\frac{T_{O}^{p}-T_{O}^{s}}{T_{O}^{p}+T_{O}^{s}}$ we get the gain ratios for the two setups $\mathrm{y}= \pm 1$ from

$$
\frac{\eta^{*}(\mathrm{y}=+1)}{\eta}=\frac{T_{O}^{s}}{T_{O}^{p}}, \quad \frac{\eta^{*}(\mathrm{y}=-1)}{\eta}=\frac{T_{O}^{p}}{T_{O}^{s}}
$$

As there are no calibrator-induced rotational errors $\varepsilon$, all equations for the standard measurements of Sect. 4 are with $\varepsilon=$ $0^{\circ}$.

\section{Calibration with a rotator - mechanical or by $\lambda / 2$ plate (HWP)}

With an ideal HWP rotator the input Stokes vector is rotated with respect to the coordinate system, while with the mechanical rotator the polarising beam splitter and, if so, the receiving optics are rotated in the opposite direction to achieve the same effect. Mathematically the latter means a rotation of the coordinate system (see Sect. S5). Furthermore, the rotation with a HWP includes a retardance of $180^{\circ}$ and hence a mirroring of the input Stokes vector (see Eq. 
S.10.13.2). We combine the two methods in the rotator matrix $\mathbf{M}_{r o t}$ (Supplement Sect. S10.15) by introducing the rotator operator $\mathrm{h}$ (Eq. S.10.15.1), which is $\mathrm{h}=+1$ for the mechanical rotator and $\mathrm{h}=-1$ for the HWP rotator.

\subsection{Calibration with a rotator before the polarising beam splitter}

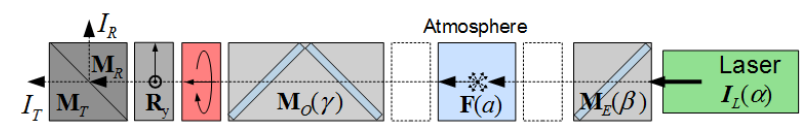

The general formula for the output Stokes vector $\boldsymbol{I}_{S}$ with a rotation calibrator $\mathbf{M}_{r o t}$ before the polarising beam splitter is Eq. (97).

$$
\begin{aligned}
& \boldsymbol{I}_{S}=\eta_{S} \mathbf{M}_{S} \mathbf{R}_{\mathrm{y}} \mathbf{M}_{r o t}\left(\mathrm{x} 45^{\circ}+\varepsilon, \mathrm{h}\right) \mathbf{M}_{o}(\gamma) \mathbf{F}(a) \mathbf{M}_{E}(\beta) \boldsymbol{I}_{L}(\alpha)= \\
& =\eta_{S} \mathbf{A}_{S}(\mathrm{y}) \mathbf{M}_{r o t}\left(\mathrm{x} 45^{\circ}+\varepsilon, \mathrm{h}\right) \boldsymbol{I}_{i n}(\gamma, a, \beta, \alpha)
\end{aligned}
$$

With the analyser part $\mathbf{A}_{S}$ from Eq. (D.5), $\mathbf{M}_{r o t}$ from Eq. (S.10.15.1), and the input Stokes vector $\boldsymbol{I}_{\text {in }}$ from 0 we get Eq. (98) for the calibration signals, and with the expanded input Stokes vector Eq. (E31) we get from Eq. (98) the general calibration signals in Eq. (99).

$$
\begin{aligned}
& \frac{I_{S}}{\eta_{S} T_{S} T_{r o t} I_{\text {in }}}=\frac{\left\langle\mathbf{M}_{S} \mathbf{R}_{\mathrm{y}}\left|\mathbf{M}_{r o t}\left(\mathrm{x} 45^{\circ}+\varepsilon, \mathrm{h}\right)\right| \boldsymbol{I}_{\text {in }}\right\rangle}{T_{S} T_{r o t} I_{\text {in }}}= \\
& =\left\langle\begin{array}{c}
1 \\
\mathrm{y} D_{S} \\
0 \\
0
\end{array}\left|\left(\begin{array}{cccc}
1 & 0 & 0 & 0 \\
0 & -\mathrm{xs}_{2 \varepsilon} & -\mathrm{xhc}_{2 \varepsilon} & 0 \\
0 & \mathrm{xc}_{2 \varepsilon} & -\mathrm{xhs}_{2 \varepsilon} & 0 \\
0 & 0 & 0 & \mathrm{~h}
\end{array}\right)\right| \begin{array}{l}
i_{i n} \\
q_{i n} \\
u_{i n} \\
v_{\text {in }}
\end{array}\right\rangle=\left\langle\begin{array}{c}
1 \\
-\mathrm{xys}_{2 \varepsilon} D_{S} \\
-\mathrm{xyhc}_{2 \varepsilon} D_{S} \\
0
\end{array} \| \begin{array}{l}
i_{i n} \\
q_{i n} \\
u_{i n} \\
v_{\text {in }}
\end{array}\right\rangle= \\
& =i_{i n}-\mathrm{xy} D_{S}\left(\mathrm{~s}_{2 \varepsilon} q_{i n}+\mathrm{hc}_{2 \varepsilon} u_{i n}\right) \\
& \frac{I_{S}}{\eta_{S} T_{S} T_{r o t} T_{O} F_{11} T_{E} I_{L}}=\frac{\left\langle\mathbf{M}_{S} \mathbf{R}_{\mathrm{y}}\left|\mathbf{M}_{r o t}\left(\mathrm{x} 45^{\circ}+\varepsilon, \mathrm{h}\right)\right| \mathbf{M}_{O}(\gamma) \mathbf{F}(a) \boldsymbol{I}_{E}\right\rangle}{T_{S} T_{r o t} T_{O} F_{11} T_{E} I_{L}}= \\
& =i_{E}+a D_{O}\left(\mathrm{c}_{2 \gamma} q_{E}-\mathrm{s}_{2 \gamma} u_{E}\right)-\mathrm{xy} D_{S}\left\{\begin{array}{l}
\mathrm{s}_{2 \varepsilon+\mathrm{h} 2 \gamma} D_{O} i_{E}+a\left(\mathrm{~s}_{2 \varepsilon} q_{E}-\mathrm{hc}_{2 \varepsilon} u_{E}\right)+ \\
+\mathrm{hc}_{2 \varepsilon+\mathrm{h} 2 \gamma}\left[W_{O} a\left(\mathrm{~s}_{2 \gamma} q_{E}+\mathrm{c}_{2 \gamma} u_{E}\right)+Z_{O} \mathrm{~s}_{O}(1-2 a) v_{E}\right]
\end{array}\right\}
\end{aligned}
$$

Since $i_{\text {in }}$ in Eq. (98) is independent of $\varepsilon, \mathrm{x}$, and $\mathrm{y}$, we can define the function $E$ in Eq. (100) and get for the calibration signals in Eq. (101) and for the gain ratios $\eta^{*}$ (Sect. 5) Eq. (102). 


$$
\begin{aligned}
& E(\varepsilon, \mathrm{h}, \gamma, a, \beta, \alpha) \equiv \frac{\mathrm{s}_{2 \varepsilon} q_{i n}+\mathrm{hc}_{2 \varepsilon} u_{i n}}{i_{i n}}= \\
& =\frac{\mathrm{s}_{2 \varepsilon+\mathrm{h} 2 \gamma} D_{O} i_{E}+a\left(\mathrm{~s}_{2 \varepsilon} q_{E}-\mathrm{hc}_{2 \varepsilon} u_{E}\right)+\mathrm{hc}_{2 \varepsilon+\mathrm{h} 2 \gamma}\left[W_{O} a\left(\mathrm{~s}_{2 \gamma} q_{E}+\mathrm{c}_{2 \gamma} u_{E}\right)+Z_{O} \mathrm{~s}_{O}(1-2 a) v_{E}\right]}{i_{E}+a D_{O}\left(\mathrm{c}_{2 \gamma} q_{E}-\mathrm{s}_{2 \gamma} u_{E}\right)} \\
& I_{S}=\eta_{S} T_{S} T_{r o t} I_{i n}\left[1-\mathrm{xy} D_{S} E\right] i_{i n} \\
& \eta^{*}=\frac{I_{R}}{I_{T}}=\frac{\eta_{R} T_{R}}{\eta_{T} T_{T}} \frac{1-\mathrm{xy} D_{R} E}{1-\mathrm{xy} D_{T} E}=\eta \frac{1-\mathrm{xy} D_{R} E}{1-\mathrm{xy} D_{T} E}
\end{aligned}
$$

Equation (103) shows the gain ratio from the $\Delta 90$ calibration, assuming that the polarisation parameter $a$ does not change in the calibration range between the two calibration measurements, i.e. $E_{+}=E_{-}$(see Sect. 5).

$$
\frac{\eta_{\Delta 90}^{*}}{\eta}=\sqrt{\frac{1-\mathrm{y} D_{R} E_{+}}{1-\mathrm{y} D_{T} E_{+}} \frac{1+\mathrm{y} D_{R} E_{-}}{1+\mathrm{y} D_{T} E_{-}}}=\sqrt{\frac{1-D_{R}{ }^{2} E^{2}}{1-D_{T}{ }^{2} E^{2}}}
$$

Special cases: We immediately see that it is advantageous to use a cleaned analyser (see Supplement Sect. S10.10) because with $D_{T}=1, D_{R}=-1$ Eq. (102) becomes Eq. (104) and all possible errors in the $\Delta 90$ calibration from Eq. (103) are removed in Eq. (105), besides the problem of temporal change of $a$.

$$
\begin{aligned}
& D_{T}=+1, D_{R}=-1 \Rightarrow \\
& \frac{\eta^{*}}{\eta}=\frac{1+\mathrm{xy} E}{1-\mathrm{xy} E} \\
& \frac{\eta_{\Delta 90}^{*}}{\eta}=\sqrt{\frac{1-E^{2}}{1-E^{2}}}=1 \Rightarrow \eta=\eta_{\Delta 90}^{*}
\end{aligned}
$$

From Eq. (100) we get Eq. (106) without emitter and receiver optics rotation, without laser rotation, but with calibrator rotation $\varepsilon$ and with a horizontally linearly polarised laser $\boldsymbol{I}_{\boldsymbol{L}}$ (Eq. E5).

$$
\begin{aligned}
& \gamma=\beta=\alpha=0 \wedge \boldsymbol{I}_{E}=\boldsymbol{I}_{L}=I_{L}\left|\begin{array}{llll}
1 & 1 & 0 & 0
\end{array}\right\rangle \Rightarrow \\
& E(\varepsilon, \mathrm{h}, 0, a, 0,0)=\mathrm{s}_{2 \varepsilon} \frac{D_{O}+a}{1+a D_{O}}
\end{aligned}
$$

If additionally without calibrator rotation error $\varepsilon$, Eq. (106) becomes Eq. (107) and thus $\eta^{*}$ and $\eta^{*} \Delta 90$ are independent of the atmospheric polarisation parameter $a$ and any atmospheric changes (see Eqs. 102 and 103).

$$
\begin{aligned}
& \varepsilon=0 \Rightarrow \\
& E(0, \mathrm{~h}, 0, a, 0,0)=0
\end{aligned}
$$


A more general case without receiver optics rotation $\gamma$ and without calibrator rotation $\varepsilon$, but with unknown laser and emitter optics rotation, Eq. (100) becomes Eq. (108).

with $\gamma=\varepsilon=0 \Rightarrow E(0, \mathrm{~h}, 0, a, \beta, \alpha)=\frac{u_{i n}}{i_{\text {in }}}=\frac{\mathrm{h} Z_{O}\left[\mathrm{~s}_{O}(1-2 a) v_{E}-\mathrm{c}_{O} a u_{E}\right]+(\mathrm{h}-1) a u_{E}}{i_{E}+a D_{O} q_{E}}$

Equation (108) remains quite complex if we use $\boldsymbol{I}_{E}$ with rotated emitter optics (Eq. E12), and even if we assume a linearly polarised laser (Eq. E9).

With a horizontally linearly polarised laser (Eq. E13) aligned with the rotated emitter optics $(\alpha=\beta)$ we get from Eq. (100)

with $\alpha=\beta \wedge \boldsymbol{I}_{E}=T_{E} I_{L}\left(1+D_{E}\right)\left|1 \quad \mathrm{c}_{2 \alpha} \quad \mathrm{s}_{2 \alpha} \quad 0\right\rangle \Rightarrow$

$E(\varepsilon, \mathrm{h}, \gamma, a, \alpha, \alpha)=\frac{\mathrm{s}_{2 \varepsilon+\mathrm{h} 2 \gamma} D_{O}+a\left[\left(\mathrm{~s}_{2 \varepsilon} \mathrm{c}_{2 \alpha}-\mathrm{hc}_{2 \varepsilon} \mathrm{s}_{2 \alpha}\right)+\mathrm{hc}_{2 \varepsilon+\mathrm{h} 2 \gamma} W_{O}\left(\mathrm{~s}_{2 \gamma} \mathrm{c}_{2 \alpha}+\mathrm{c}_{2 \gamma} \mathrm{s}_{2 \alpha}\right)\right]}{1+a D_{O}\left(\mathrm{c}_{2 \gamma} \mathrm{c}_{2 \alpha}-\mathrm{s}_{2 \gamma} \mathrm{s}_{2 \alpha}\right)}=$

$=\frac{\mathrm{s}_{2 \varepsilon+\mathrm{h} 2 \gamma} D_{O}+a\left(\mathrm{hc}_{2 \varepsilon+\mathrm{h} 2 \gamma} \mathrm{s}_{2 \gamma+2 \alpha} W_{O}+\mathrm{s}_{2 \varepsilon-\mathrm{h} 2 \alpha}\right)}{1+a D_{O} \mathrm{c}_{2 \gamma+2 \alpha}}$

Note that $D_{E}=0$ means without emitter optics, and $W_{O}=\left(1-Z_{O} \mathrm{c}_{O}\right)$.

Equation (109) with laser, emitter and receiver optics aligned with each other becomes

with $\alpha=\beta=-\gamma \wedge \boldsymbol{I}_{E}=T_{E} I_{L}\left(1+D_{E}\right)\left|1 \quad \mathrm{c}_{2 \alpha} \quad \mathrm{s}_{2 \alpha} \quad 0\right\rangle \Rightarrow$

$E(\varepsilon, \mathrm{h}, \gamma, a,-\gamma,-\gamma)=\mathrm{s}_{2 \varepsilon+\mathrm{h} 2 \gamma} \frac{D_{O}+\mathrm{h} a}{1+a D_{O}}$

Equation (109) with receiver optics and calibrator aligned becomes

with $\alpha=\beta, \varepsilon=-\mathrm{h} \gamma \wedge \boldsymbol{I}_{E}=T_{E} I_{L}\left(1+D_{E}\right)\left|1 \quad \mathrm{c}_{2 \alpha} \quad \mathrm{s}_{2 \alpha} \quad 0\right\rangle \Rightarrow$

$E(-\gamma, \mathrm{h}, \gamma, a, \alpha, \alpha)=\frac{\mathrm{hs}_{2 \gamma+2 \alpha} a\left(1-Z_{O} \mathrm{c}_{O}-\mathrm{h}\right)}{1+a D_{O} \mathrm{c}_{2 \gamma+2 \alpha}}$

In summary, the $\Delta 90$ calibration with a cleaned analyser results in a calibration factor $\eta$ independent of $\boldsymbol{I}_{i n}$, i.e. independent of any optics before the calibrator and independent of the rotation error $\varepsilon$ of the calibrator. Calibrations without a cleaned analyser include error terms which increase rapidly with increasing $\varepsilon$ and $\alpha$ for the individual $\pm 45^{\circ}$ calibrations (Bravo-Aranda et al., 2016) because $D_{T}$ and $D_{R}$ in the numerator and denominator have opposite signs in Eq. (102). The geometric mean of the two $\pm 45^{\circ}$ calibrations in Eq. (103) removes the opposite signs and the increasing error with increasing $\varepsilon$ and $\alpha$ is reduced by orders of magnitude compared to the individual $\pm 45^{\circ}$ calibrations (Freudenthaler et al., 2009). 
7.2 Calibration with a rotator before the receiving optics

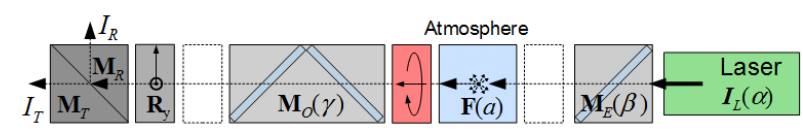

The general formula for the output Stokes vector $\boldsymbol{I}_{S}$ with rotation calibrator before the receiving optics $\mathbf{M}_{O}$ and the polarising beam splitter $\mathbf{M}_{S}$ is given in Eq. (112).

$$
\begin{aligned}
& \boldsymbol{I}_{S}=\eta_{S} \mathbf{M}_{S} \mathbf{R}_{\mathrm{y}} \mathbf{M}_{o}(\gamma) \mathbf{M}_{r o t}\left(\mathrm{x} 45^{\circ}+\varepsilon, \mathrm{h}\right) \mathbf{F}(a) \mathbf{M}_{E}(\beta) \boldsymbol{I}_{L}(\alpha)= \\
& =\eta_{S} \mathbf{A}_{S}(\mathrm{y}, \gamma) \mathbf{M}_{r o t}\left(\mathrm{x} 45^{\circ}+\varepsilon, \mathrm{h}\right) \boldsymbol{I}_{i n}(a, \beta, \alpha)
\end{aligned}
$$

With $\mathbf{A}_{S}$ from Eq. (D7), $\mathbf{M}_{r o t}$ from Eq. (S.10.15.1), and $\boldsymbol{I}_{\text {in }}$ from Sect. E3, i.e. Eq. (E19), we get Eq. (113) for the calibration signals using the trigonometric relations in Supplement Sect. S12.

$$
\begin{aligned}
& \frac{I_{S}}{\eta_{S} T_{S} T_{O} T_{r o t} F_{11} T_{E} I_{L}}=\frac{\left\langle\mathbf{M}_{S} \mathbf{R}_{\mathrm{y}} \mathbf{M}_{O}(\gamma)\left|\mathbf{M}_{r o t}\left(\mathrm{x} 45^{\circ}+\varepsilon, \mathrm{h}\right)\right| \mathbf{F}(a) \boldsymbol{I}_{E}\right\rangle}{T_{S} T_{O} T_{r o t} F_{11} T_{E} I_{L}}= \\
& \left.=\left(\begin{array}{c}
1+\mathrm{yc}_{2 \gamma} D_{O} D_{S} \\
\mathrm{c}_{2 \gamma} D_{O}+\mathrm{y}_{S}\left(1-\mathrm{s}_{2 \gamma}^{2} W_{O}\right) \\
\mathrm{s}_{2 \gamma}\left(D_{O}+\mathrm{yc}_{2 \gamma} D_{S} W_{O}\right) \\
-\mathrm{ys}_{2 \gamma} D_{S} Z_{O} \mathrm{~s}_{O}
\end{array} \mid \begin{array}{cccc}
1 & 0 & 0 & 0 \\
0 & -\mathrm{xs}_{2 \varepsilon} & -\mathrm{xhc}_{2 \varepsilon} & 0 \\
0 & \mathrm{xc}_{2 \varepsilon} & -\mathrm{xhs}_{2 \varepsilon} & 0 \\
0 & 0 & 0 & \mathrm{~h}
\end{array}\right) \mid \begin{array}{c}
i_{E} \\
a q_{E} \\
-a u_{E} \\
(1-2 a) v_{E}
\end{array}\right)= \\
& =\left(1+\mathrm{yc}_{2 \gamma} D_{O} D_{S}\right) i_{E}-\mathrm{yhs}_{2 \gamma} D_{S} Z_{O} \mathrm{~s}_{O}(1-2 a) v_{E}+ \\
& -\mathrm{x} a\left\{D_{O}\left(q_{E} \mathrm{~s}_{2 \varepsilon-2 \gamma}-\mathrm{h} u_{E} \mathrm{c}_{2 \varepsilon-2 \gamma}\right)-\mathrm{y} D_{S}\left[\mathrm{~s}_{2 \gamma} W_{O}\left(q_{E} \mathrm{c}_{2 \varepsilon-2 \gamma}+\mathrm{h} u_{E} \mathrm{~s}_{2 \varepsilon-2 \gamma}\right)-\left(q_{E} \mathrm{~s}_{2 \varepsilon}-\mathrm{h} u_{E} \mathrm{c}_{2 \varepsilon}\right)\right]\right\}
\end{aligned}
$$

Special cases: Without receiver optics rotation, i.e. $\gamma=0$, Eq. (113) becomes Eq. (114), which is less complex and independent of retardation terms $Z_{O} \mathrm{~s}_{O}$ and $W_{O}$, and the gain ratios $\eta^{*}$ (Sect. 5) can be written as Eqs. (115) and (116).

$$
\begin{aligned}
& \gamma=0 \Rightarrow \\
& I_{S} /\left(\eta_{S} T_{S} T_{O} T_{r o t} F_{11} T_{E} I_{L}\right)=\left(1+\mathrm{y} D_{O} D_{S}\right) i_{E}-\mathrm{x} a\left(D_{O}+\mathrm{y} D_{S}\right)\left(q_{E} \mathrm{~s}_{2 \varepsilon}-\mathrm{h} u_{E} \mathrm{c}_{2 \varepsilon}\right) \\
& \frac{\eta^{*}}{\eta}=\frac{\left(1+\mathrm{y} D_{O} D_{R}\right) i_{E}-\mathrm{x} a\left(D_{O}+\mathrm{y} D_{R}\right)\left(q_{E} \mathrm{~s}_{2 \varepsilon}-\mathrm{h} u_{E} \mathrm{c}_{2 \varepsilon}\right)}{\left(1+\mathrm{y} D_{O} D_{T}\right) i_{E}-\mathrm{x} a\left(D_{O}+\mathrm{y} D_{T}\right)\left(q_{E} \mathrm{~s}_{2 \varepsilon}-\mathrm{h} u_{E} \mathrm{c}_{2 \varepsilon}\right)} \\
& \frac{\eta_{\Delta 90}^{*}}{\eta}=\sqrt{\frac{\left(1+\mathrm{y} D_{O} D_{R}\right)^{2} i_{E}{ }^{2}-\left(D_{O}+\mathrm{y} D_{R}\right)^{2}\left(q_{E} \mathrm{~s}_{2 \varepsilon}-\mathrm{h} u_{E} \mathrm{c}_{2 \varepsilon}\right)^{2}}{\left(1+\mathrm{y} D_{O} D_{T}\right)^{2} i_{E}{ }^{2}-\left(D_{O}+\mathrm{y} D_{T}\right)^{2}\left(q_{E} \mathrm{~s}_{2 \varepsilon}-\mathrm{h} u_{E} \mathrm{c}_{2 \varepsilon}\right)^{2}}}
\end{aligned}
$$

With a cleaned analyser (see Supplement Sect. S10.10) Eqs. (115) and (116) become Eqs. (117) and (118). 


$$
\begin{aligned}
& \gamma=0^{\circ}, D_{T}=+1, D_{R}=-1 \Rightarrow \\
& \frac{\eta^{*}}{\eta}=\frac{1-\mathrm{y} D_{O}}{1+\mathrm{y} D_{O}} \frac{i_{E}+\mathrm{xy} a\left(q_{E} \mathrm{~s}_{2 \varepsilon}-\mathrm{h} u_{E} \mathrm{c}_{2 \varepsilon}\right)}{i_{E}-\mathrm{xy} a\left(q_{E} \mathrm{~s}_{2 \varepsilon}-\mathrm{h} u_{E} \mathrm{c}_{2 \varepsilon}\right)} \\
& \frac{\eta_{\Delta 90}^{*}}{\eta}=\frac{1-\mathrm{y} D_{O}}{1+\mathrm{y} D_{O}}
\end{aligned}
$$

The gain ratio $\eta^{*}{ }_{\Delta 90}$ in Eq. (118) is independent of the input Stokes vector, i.e. the laser polarisation, independent of the calibrator type (mechanical or $\lambda / 2$ plate rotation) and of the calibrator rotation $\varepsilon$. Using the two calibration setups Eqs. (118) (before the receiving optics) and (105) (before the PBS) it is possible to retrieve the receiver optics diattenuation parameter $D_{O}$. Furthermore, with this setup and the measured gain ratio $\eta^{*}{ }_{\Delta 90}$ from Eq. (118) we get the polarisation parameter $a$ (Eq. 119) and the backscatter coefficient $F_{1 l}$ (Eq. 120) with Eq. (78) directly from the measurement signals $I_{R}$ and $I_{T}$ according to Eqs. (61) and (65) without the explicit knowledge of $D_{O}$ or any other correction.

$$
\begin{gathered}
a=\mathrm{y} \frac{\eta_{\Delta 90}^{*} I_{T}-I_{R}}{\eta_{\Delta 90}^{*} I_{T}+I_{R}} \\
F_{11} \propto \eta_{\Delta 90}^{*} I_{T}+I_{R}
\end{gathered}
$$

\subsection{Calibration with a rotator behind the emitter optics}

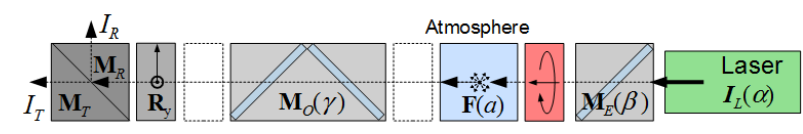

The general formula for the output Stokes vector $\boldsymbol{I}_{S}$ with rotation calibrator $\mathbf{M}_{r o t}$ (Eq. S.10.15.2) behind the emitter optic $\mathbf{M}_{E}$ and all derivations therefrom can be derived from Sect. 7.2 using Eq. (121) and considering the mirror effect of $\mathbf{F}$ and the associated sign changes in the rotation angle (Supplement Sect. S6.3) when mathematically moving the calibrator $\mathbf{M}_{\text {rot }}$ from behind the emitter optics $\mathbf{M}_{E}$ to before the receiving optics $\mathbf{M}_{O}$. Regarding the rotation and mirror relations see Supplement Sects. S5 and S6.

$$
\begin{aligned}
& \boldsymbol{I}_{S}=\eta_{S} \mathbf{M}_{S} \mathbf{R}_{\mathrm{y}} \mathbf{M}_{o}(\gamma) \mathbf{F}(a) \mathbf{M}_{r o t}\left(\mathrm{x} 45^{\circ}+\varepsilon, \mathrm{h}\right) \mathbf{M}_{E}(\beta) \boldsymbol{I}_{L}(\alpha)= \\
& =\eta_{S} \mathbf{M}_{S} \mathbf{R}_{\mathrm{y}} \mathbf{M}_{o}(\gamma) \mathbf{F}(a) \mathbf{R}\left(\mathrm{x} 45^{\circ}\right) \mathbf{R}(\varepsilon) \mathbf{M}_{\mathrm{h}} \mathbf{M}_{E}(\beta) \boldsymbol{I}_{L}(\alpha)= \\
& =\eta_{S} \mathbf{M}_{S} \mathbf{R}_{\mathrm{y}} \mathbf{M}_{o}(\gamma) \mathbf{R}\left(-\mathrm{x} 45^{\circ}\right) \mathbf{R}(-\varepsilon) \mathbf{M}_{\mathrm{h}} \mathbf{F}(a) \mathbf{M}_{E}(\beta) \boldsymbol{I}_{L}(\alpha) \\
& =\eta_{S} \mathbf{M}_{S} \mathbf{R}_{\mathrm{y}} \mathbf{M}_{o}(\gamma) \mathbf{M}_{r o t}\left(-\mathrm{x} 45^{\circ}-\varepsilon, \mathrm{h}\right) \mathbf{F}(a) \mathbf{M}_{E}(\beta) \boldsymbol{I}_{L}(\alpha)
\end{aligned}
$$

\section{Calibration with a linear polariser $(\mathbf{P})$}

A linear polariser is a retarding linear diattenuator (Supplement Sect. S10.3). The output of an ideal linear polariser is linearly polarised light independent of the state of polarisation of the input, which seems to be ideal for our purpose. 
Polarising sheet filters are thin and have large acceptance angles. Hence they can be easily included in existing lidar systems, even in diverging or converging light paths as close to the telescope focus. However, to achieve an acceptable uncertainty of the calibration factor, a rather good extinction ratio of the linear polariser of the order of $10^{-4}$ and better is necessary. Crystal polarisers exhibit such high extinction ratios, but the available diameters are limited, are bulky, and have smaller acceptance angles. Wire grid and liquid crystal polarisers usually do not show high enough extinction ratios. A linear polariser is described in the same way as a polarising beam splitter, which is a retarding diattenuator (Supplement Sect. S4 and S10.3ff.) with high diattenuation $\left(\mathbf{D}_{P} \approx 1\right)$. Since the standard measurements have to be performed without the linearly polarising calibrator, there is no rotational misalignment $\varepsilon$ for the standard measurement signals of Sect. 4. As the equations become too complex with a real linear polariser with diattenuation and retardation, we use a real linear polariser only in Sect. 8.1 to show as an example how the uncertainty of the extinction ratio influences the accuracy of the calibration factor, and otherwise we use an ideal linear polariser. The general formula with a real linear polariser can be found in Sect. C2.

\subsection{Calibration with a linear polariser before the polarising beam splitter}

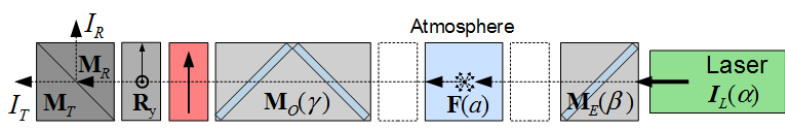

$$
\begin{aligned}
& \boldsymbol{I}_{S}=\eta_{S} \mathbf{M}_{S} \mathbf{R}(\mathrm{y}) \mathbf{M}_{P}\left(\mathrm{x} 45^{\circ}+\varepsilon\right) \mathbf{M}_{o}(\gamma) \mathbf{F}(a) \mathbf{M}_{E}(\beta) \boldsymbol{I}_{L}(\alpha)= \\
& =\eta_{S} \mathbf{A}_{S}(\mathrm{y}) \mathbf{M}_{P}\left(\mathrm{x} 45^{\circ}+\varepsilon\right) \boldsymbol{I}_{i n}(\gamma, a, \beta, \alpha)
\end{aligned}
$$

With Eq. (D5) for the analyser part $\mathbf{A}_{S}$, Eq. (S.10.6.1) for the rotated linear polariser, and $\boldsymbol{I}_{\text {in }}$ from Sect. E4 we get the general calibration signals in Eq. (123).

$$
\begin{aligned}
& \frac{I_{S}}{\eta_{S} T_{S} T_{P} I_{\text {in }}}=\frac{\left\langle\mathbf{M}_{S} \mathbf{R}_{\mathrm{y}}\left|\mathbf{M}_{P}\left(\mathrm{x} 45^{\circ}+\varepsilon\right)\right| \mathbf{I}_{\text {in }}\right\rangle}{T_{S} T_{P} I_{\text {in }}}= \\
& =\left\langle\begin{array}{c|c}
1-\mathrm{xys}_{2 \varepsilon} D_{P} D_{S} \\
-\mathrm{xs}_{2 \varepsilon} D_{P}+\mathrm{y}_{S}\left(1-\mathrm{c}_{2 \varepsilon}^{2} W_{P}\right) \\
\mathrm{xc}_{2 \varepsilon} D_{P}-\mathrm{ys}_{2 \varepsilon} \mathrm{c}_{2 \varepsilon} W_{P} D_{S} \\
-\mathrm{xyc}_{2 \varepsilon} Z_{P} \mathrm{~s}_{P} D_{S}
\end{array} \mid \begin{array}{l}
i_{\text {in }} \\
q_{\text {in }} \\
u_{\text {in }} \\
v_{\text {in }}
\end{array}\right\rangle= \\
& =i_{\text {in }}+\mathrm{y} D_{S}\left[q_{\text {in }}-\mathrm{c}_{2 \varepsilon} W_{P}\left(\mathrm{c}_{2 \varepsilon} q_{\text {in }}+\mathrm{s}_{2 \varepsilon} u_{\text {in }}\right)\right]- \\
& -\mathrm{x}\left[D_{P}\left(\mathrm{~s}_{2 \varepsilon} q_{i n}-\mathrm{c}_{2 \varepsilon} u_{\text {in }}\right)+\mathrm{y} D_{S}\left(\mathrm{~s}_{2 \varepsilon} D_{P} i_{i n}+\mathrm{c}_{2 \varepsilon} Z_{P} \mathrm{~s}_{P} v_{i n}\right)\right]
\end{aligned}
$$

Special cases: Without calibrator rotation error $\varepsilon$ Eq. (123) becomes Eq. (124). 
$\varepsilon=0 \Rightarrow$

$\frac{I_{S}}{\eta_{S} T_{S} T_{P} I_{\text {in }}}=i_{\text {in }}+\mathrm{y} D_{S}\left[1-W_{P}\right] q_{i n}+\mathrm{x}\left[u_{i n} D_{P}-\mathrm{y} D_{S} Z_{P} \mathrm{~s}_{P} v_{i n}\right]=$

$=i_{\text {in }}+\mathrm{x} D_{P} u_{\text {in }}+\mathrm{y} D_{S} Z_{P}\left(\mathrm{c}_{P} q_{\text {in }}-\mathrm{s}_{P} v_{\text {in }}\right)$

We get with a cleaned analyser and horizontally linearly polarised input $\boldsymbol{I}_{\text {in }}$ with Eq. (124) the gain ratios (Sect. 5) in Eq. (125).

$\varepsilon=0, D_{T}=+1, D_{R}=-1, \boldsymbol{I}_{\text {in }}=\left|\begin{array}{llll}1 & 1 & 0 & 0\end{array}\right\rangle \Rightarrow$

$\frac{\eta^{*}}{\eta}=\frac{1-\mathrm{y} Z_{P}}{1+\mathrm{y} Z_{P}}$

Using Eq. (S.10.10.8) for the extinction ratio $\rho$ of the real linear polariser, we get the approximation Eq. (126) for the gain ratios depending on $\rho$, with which we can estimate the error of the gain ratio if we use a real polariser with extinction ratio $\rho$ for the measurements but assume an ideal polariser as a calibrator in the correction equations. Equation (126) with $\rho=10^{-5}$ and $\rho=10^{-4}$, for example, gives relative errors of the gain ratios of about 1.3 and $8 \%$, respectively.

with $\rho=k_{2} / k_{1}$ and $k_{2} \ll k_{1} \Rightarrow$

$\frac{\eta^{*}}{\eta} \approx \frac{1-2 \mathrm{y} \sqrt{\rho}}{1+2 \mathrm{y} \sqrt{\rho}} \approx 1-4 \mathrm{y} \sqrt{\rho}$

With an ideal linear polariser Eq. (123) becomes Eq. (127), and the gain ratios in Eq. (128) are independent of $\boldsymbol{I}_{\text {in }}$, i.e. independent of the laser polarisation, of the atmospheric depolarisation, and of any optics before the calibrator. The error due to the calibrator rotation $\varepsilon$ is largely reduced with the $\Delta 90$ calibration in Eq. (129) compared to the $\pm 45^{\circ}$ calibration in Eq. (128).

$D_{P}=1 \Rightarrow W_{P}=1, Z_{P}=0 \Rightarrow$

$\frac{I_{S}}{\eta_{S} T_{S} T_{P} I_{\text {in }}}=\left(1-\mathrm{xys}_{2 \varepsilon} D_{S}\right)\left\langle 1 \quad-\mathrm{xs}_{2 \varepsilon} \quad \mathrm{xc}_{2 \varepsilon} \quad 0 \mid i_{\text {in }} \quad q_{\text {in }} \quad u_{\text {in }} \quad v_{\text {in }}\right\rangle=$

$=\left(1-\mathrm{xys}_{2 \varepsilon} D_{S}\right)\left[i_{i n}-\mathrm{x}\left(\mathrm{s}_{2 \varepsilon} q_{i n}-\mathrm{c}_{2 \varepsilon} u_{i n}\right)\right]$

$\frac{\eta^{*}}{\eta}=\frac{1-\operatorname{xys}_{2 \varepsilon} D_{R}}{1-\operatorname{xys}_{2 \varepsilon} D_{T}}$

$\frac{\eta_{\Delta 90}^{*}}{\eta}=\sqrt{\frac{1-\mathrm{s}_{2 \varepsilon}{ }^{2} D_{R}{ }^{2}}{1-\mathrm{s}_{2 \varepsilon}{ }^{2} D_{T}{ }^{2}}}$ 
If additionally a cleaned analyser is used (see Supplement Sect. S10.10), Eqs. (128) and (129) become Eqs. (130) and (131). Equation (130) is of the form of Eq. (193) and can be used to determine $\varepsilon$ (see Sect. 11). Equation (131) shows that the $\Delta 90$ calibration with a cleaned analyser is free of $\varepsilon$ error.

with $D_{P}=1, W_{P}=1, Z_{P}=0, D_{T}=+1, D_{R}=-1 \Rightarrow$

$\frac{\eta^{*}}{\eta}=\frac{1+\mathrm{xys}_{2 \varepsilon}}{1-\mathrm{xys}_{2 \varepsilon}}$

$\eta_{\Delta 90}^{*}=\eta=\frac{\eta_{R} T_{R}^{s}}{\eta_{T} T_{T}^{p}}$

\subsection{Calibration with an ideal linear polariser before the receiving optics}

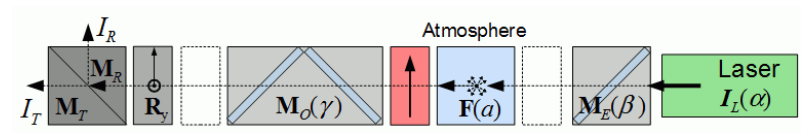

$\boldsymbol{I}_{S}=\eta_{S} \mathbf{M}_{S} \mathbf{R}(\mathrm{y}) \mathbf{M}_{o}(\gamma) \mathbf{M}_{P}\left(\mathrm{x} 45^{\circ}+\varepsilon\right) \mathbf{F}(a) \mathbf{M}_{E}(\beta) \boldsymbol{I}_{L}(\alpha)=$

$=\eta_{S} \mathbf{A}_{S}(\mathrm{y}, \gamma) \mathbf{M}_{P}\left(\mathrm{x} 45^{\circ}+\varepsilon\right) \boldsymbol{I}_{i n}(a, \beta, \alpha)$

With Eq. (D.7) for the analyser part $\mathbf{A}_{S}$, Eq. (S.10.8.6) for the ideal linear polariser $\mathbf{M}_{P}$, and any of the input Stokes vectors $\boldsymbol{I}_{\text {in }}$ of 0 we get Eq. (133) for the calibration signals $I_{S}$. Since the last term of Eq. (133) is independent of the analyser diattenuation parameters $D_{S}$, this term cancels out in the ratio of the gain ratios (Sect. 5) in Eq. (134), which are therefore independent of the input Stokes vector.

with $D_{P}=1 \Rightarrow$

$\frac{I_{S}}{\eta_{S} T_{S} T_{O} T_{P} F_{11} T_{E} I_{L}}=\frac{\left\langle\mathbf{M}_{S} \mathbf{R}_{\mathrm{y}} \mathbf{M}_{O}(\gamma)\left|\mathbf{M}_{P}\left(\mathrm{x} 45^{\circ}+\varepsilon\right)\right| \mathbf{F}(a) \boldsymbol{I}_{E}\right\rangle}{T_{S} T_{O} T_{P} F_{11} T_{E} I_{L}}=$

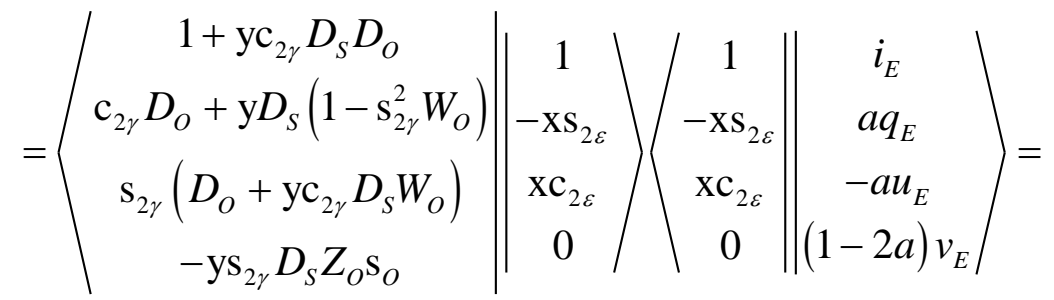

$=\left[\left(1+\mathrm{yc}_{2 \gamma} D_{S} D_{O}\right)-\mathrm{x}\left\{\mathrm{s}_{2 \varepsilon-2 \gamma} D_{O}+\mathrm{y}_{S}\left[\mathrm{~s}_{2 \varepsilon}-\mathrm{s}_{2 \gamma} \mathrm{c}_{2 \varepsilon-2 \gamma} W_{O}\right]\right\}\right]\left[i_{E}-\mathrm{x} a\left(\mathrm{~s}_{2 \varepsilon} q_{E}+\mathrm{c}_{2 \varepsilon} u_{E}\right)\right]$

$\frac{\eta^{*}}{\eta}=\frac{\left(1+\mathrm{yc}_{2 \gamma} D_{O} D_{R}\right)-\mathrm{x}\left[\mathrm{s}_{2 \varepsilon-2 \gamma} D_{O}+\mathrm{y} D_{R}\left(\mathrm{~s}_{2 \varepsilon}-\mathrm{s}_{2 \gamma} \mathrm{c}_{2 \varepsilon-2 \gamma} W_{O}\right)\right]}{\left(1+\mathrm{yc}_{2 \gamma} D_{O} D_{T}\right)-\mathrm{x}\left[\mathrm{s}_{2 \varepsilon-2 \gamma} D_{O}+\mathrm{y} D_{T}\left(\mathrm{~s}_{2 \varepsilon}-\mathrm{s}_{2 \gamma} \mathrm{c}_{2 \varepsilon-2 \gamma} W_{O}\right)\right]}$ 
Special cases: Eq. (134) does not become simple with only a cleaned analyser (Eq. 135) or without receiver optics rotation gamma (Eq. 136), but with both; i.e. with a cleaned analyser and gamma = 0, we get Eq. (137), which is of the form Eq.(193). The corresponding $\Delta 90$ calibration in Eq. (138) can be used together with the calibration measurements, which directly yield $\eta$ (see Eqs. 131 or 105, for example) to determine the diattenuation parameter $D_{O}$ of the receiving optics.

with $D_{P}=1, D_{T}=+1, D_{R}=-1 \Rightarrow$

$\frac{\eta^{*}}{\eta}=\frac{\left(1-\mathrm{yc}_{2 \gamma} D_{O}\right)-\mathrm{x}\left[\mathrm{s}_{2 \varepsilon-2 \gamma} D_{O}-\mathrm{y}\left(\mathrm{s}_{2 \varepsilon}-\mathrm{s}_{2 \gamma} \mathrm{c}_{2 \varepsilon-2 \gamma} W_{O}\right)\right]}{\left(1+\mathrm{yc}_{2 \gamma} D_{O}\right)-\mathrm{x}\left[\mathrm{s}_{2 \varepsilon-2 \gamma} D_{O}+\mathrm{y}\left(\mathrm{s}_{2 \varepsilon}-\mathrm{s}_{2 \gamma} \mathrm{c}_{2 \varepsilon-2 \gamma} W_{O}\right)\right]}$

with $D_{P}=1, \gamma=0 \Rightarrow$

$\frac{\eta^{*}}{\eta}=\frac{\left(1+\mathrm{y} D_{O} D_{R}\right)-\mathrm{xs}_{2 \varepsilon}\left[D_{O}+\mathrm{y} D_{R}\right]}{\left(1+\mathrm{y} D_{O} D_{T}\right)-\mathrm{xs}_{2 \varepsilon}\left[D_{O}+\mathrm{y} D_{T}\right]}$

with $D_{P}=1, D_{T}=+1, D_{R}=-1, \gamma=0 \Rightarrow$

$\frac{\eta^{*}}{\eta}=\frac{\left(1-\mathrm{y} D_{O}\right)-\mathrm{xs}_{2 \varepsilon}\left(D_{O}-\mathrm{y}\right)}{\left(1+\mathrm{y} D_{O}\right)-\mathrm{xs}_{2 \varepsilon}\left(D_{O}+\mathrm{y}\right)}=\frac{1-\mathrm{y} D_{O}}{1+\mathrm{y} D_{O}} \frac{1+\mathrm{xys}_{2 \varepsilon}}{1-\mathrm{xys}_{2 \varepsilon}}$

$\frac{\eta_{\Delta 90}^{*}}{\eta}=\frac{1-\mathrm{y} D_{O}}{1+\mathrm{y} D_{O}}$

\subsection{Calibration with an ideal linear polariser behind the emitter optics}

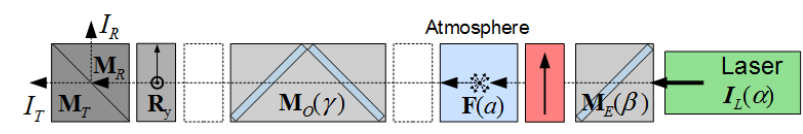

$\boldsymbol{I}_{S}=\eta_{S} \mathbf{M}_{S} \mathbf{R}_{\mathrm{y}} \mathbf{M}_{O}(\gamma) \mathbf{F}(a) \mathbf{M}_{P}\left(\mathrm{x} 45^{\circ}+\varepsilon\right) \mathbf{M}_{E}(\beta) \boldsymbol{I}_{L}(\alpha)=$

$=\eta_{S} \mathbf{A}_{S}(\mathrm{y}, \gamma, a) \mathbf{M}_{P}\left(\mathrm{x} 45^{\circ}+\varepsilon\right) \boldsymbol{I}_{i n}(\beta, \alpha)$

With Eq. (D13) for the analyser part $\mathbf{A}_{S}$, Eq. (S.10.8.6) for the ideal linear polariser $\mathbf{M}_{P}$, and any of the emitter Stokes vectors $\boldsymbol{I}_{E}$ of Sect. E2, we get the calibration signals $I_{S}$ in Eq. (140). Since the last term of Eq. (140) is independent of analyser diattenuation parameters $D_{S}$, it cancels out in the ratio of the gain ratios (Sect. 5), and the gain ratios in Eq. (141) are independent of the input Stokes vector. 
with $D_{P}=1 \Rightarrow$

$\frac{I_{S}}{\eta_{S} T_{S} T_{O} F_{11} T_{P} I_{\text {in }}}=\frac{\left\langle\mathbf{M}_{S} \mathbf{R}_{\mathrm{y}} \mathbf{M}_{O}(\gamma)\left|\mathbf{F}(a) \mathbf{M}_{P}\left(\mathrm{x} 45^{\circ}+\varepsilon\right)\right| \boldsymbol{I}_{E}\right\rangle}{T_{S} T_{O} F_{11} T_{P} I_{E}}=$

$=\left\langle\begin{array}{c}1+\mathrm{yc}_{2 \gamma} D_{S} D_{O} \\ a\left[\mathrm{c}_{2 \gamma} D_{O}+\mathrm{y}_{S}\left(1-\mathrm{s}_{2 \gamma}^{2} W_{O}\right)\right] \\ -a \mathrm{~s}_{2 \gamma}\left(D_{O}+\mathrm{yc}_{2 \gamma} D_{S} W_{O}\right) \\ -(1-2 a) \mathrm{ys}_{2 \gamma} D_{S} Z_{O} \mathrm{~s}_{O}\end{array}|| \begin{array}{c}1 \\ -\mathrm{xs}_{2 \varepsilon} \\ \mathrm{xc}_{2 \varepsilon} \\ 0\end{array}\right\rangle\left(\begin{array}{c}1 \\ -\mathrm{xs}_{2 \varepsilon} \\ \mathrm{xc}_{2 \varepsilon} \\ 0\end{array}\right\rangle=$

$=\left[\left(1+\mathrm{yc}_{2 \gamma} D_{S} D_{O}\right)-a \mathrm{x}\left\{\mathrm{s}_{2 \varepsilon+2 \gamma} D_{O}+\mathrm{y} D_{S}\left[\mathrm{~s}_{2 \varepsilon}+\mathrm{s}_{2 \gamma} \mathrm{c}_{2 \varepsilon+2 \gamma} W_{O}\right]\right\}\right]\left[i_{E}-\mathrm{x}\left(\mathrm{s}_{2 \varepsilon} q_{E}-\mathrm{c}_{2 \varepsilon} u_{E}\right)\right]$

$\frac{\eta^{*}}{\eta}=\frac{\left(1+\mathrm{yc}_{2 \gamma} D_{O} D_{R}\right)-\mathrm{xa}\left[\mathrm{s}_{2 \varepsilon+2 \gamma} D_{O}+\mathrm{y} D_{R}\left(\mathrm{~s}_{2 \varepsilon}+\mathrm{s}_{2 \gamma} \mathrm{c}_{2 \varepsilon+2 \gamma} W_{O}\right)\right]}{\left(1+\mathrm{yc}_{2 \gamma} D_{O} D_{T}\right)-\mathrm{xa}\left[\mathrm{s}_{2 \varepsilon+2 \gamma} D_{O}+\mathrm{y} D_{T}\left(\mathrm{~s}_{2 \varepsilon}+\mathrm{s}_{2 \gamma} \mathrm{c}_{2 \varepsilon+2 \gamma} W_{O}\right)\right]}$

Special cases: Eq. (141) with a cleaned analyser becomes Eq. (142), without receiver optics rotation Eq. (143), and with both conditions Eq. (144). Equation (144) is of the form of Eq. (199) and can be used to determine $\varepsilon$ (see Sect. 11). As before in Eq. (138) the corresponding $\Delta 90$ calibration becomes Eq. (145),

with $D_{P}=1, D_{T}=+1, D_{R}=-1 \Rightarrow$

$\frac{\eta^{*}}{\eta}=\frac{\left(1-\mathrm{yc}_{2 \gamma} D_{O}\right)-\mathrm{xa}\left[\mathrm{s}_{2 \varepsilon+2 \gamma} D_{O}-\mathrm{y}\left(\mathrm{s}_{2 \varepsilon}+\mathrm{s}_{2 \gamma} \mathrm{c}_{2 \varepsilon+2 \gamma} W_{O}\right)\right]}{\left(1+\mathrm{yc}_{2 \gamma} D_{O}\right)-\mathrm{xa}\left[\mathrm{s}_{2 \varepsilon+2 \gamma} D_{O}+\mathrm{y}\left(\mathrm{s}_{2 \varepsilon}+\mathrm{s}_{2 \gamma} \mathrm{c}_{2 \varepsilon+2 \gamma} W_{O}\right)\right]}$

with $D_{P}=1, \gamma=0 \Rightarrow$

$\frac{\eta^{*}}{\eta}=\frac{\left(1+\mathrm{y} D_{O} D_{R}\right)-\mathrm{xas}_{2 \varepsilon}\left[D_{O}+\mathrm{y} D_{R}\right]}{\left(1+\mathrm{y} D_{O} D_{T}\right)-\mathrm{xas}_{2 \varepsilon}\left[D_{O}+\mathrm{y} D_{T}\right]}$

with $D_{P}=1, D_{T}=+1, D_{R}=-1, \gamma=0 \Rightarrow$

$\frac{\eta^{*}}{\eta}=\frac{\left(1-\mathrm{y} D_{O}\right)-\operatorname{xas}_{2 \varepsilon}\left(D_{O}-\mathrm{y}\right)}{\left(1+\mathrm{y} D_{O}\right)-\operatorname{xas}_{2 \varepsilon}\left(D_{O}+\mathrm{y}\right)}=\frac{\left(1-\mathrm{y} D_{O}\right)\left(1+\operatorname{xyas}_{2 \varepsilon}\right)}{\left(1+\mathrm{y} D_{O}\right)\left(1-\operatorname{xyas}_{2 \varepsilon}\right)}$

$\frac{\eta_{\Delta 90}^{*}}{\eta}=\frac{1-\mathrm{y} D_{O}}{1+\mathrm{y} D_{O}}$

\section{Calibration with a $\lambda / 4$ plate (QWP)}

A $\lambda / 4$ plate (QWP) is a retarding linear diattenuator (Supplement Sect. S4) with $90^{\circ}$ phase shift between the polarisation parallel and perpendicular to the fast axis and without diattenuation (Sect. S10.16ff). Further details can be found in Bennett (2009a), Bennett (2009b), and Chipman (2009b). Oriented at $\pm 45^{\circ}$ relative to the incident linear polarisation, its output is circularly polarised. Since the equations with a real QWP with retardation error $\omega$ (Supplement Sect. S10.16) 
are too complex, we consider $\omega$ only in Sect. 9.1 to show with an example how this uncertainty influences the accuracy of the calibration factor. The general formula with a real QWP can be found in Sect. C3.

\subsection{Calibration with a $\lambda / 4$ plate before the polarising beam splitter}

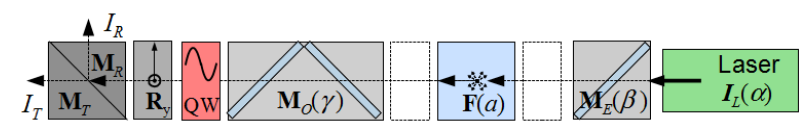

$$
\begin{aligned}
& \boldsymbol{I}_{S}=\eta_{S} \mathbf{M}_{S} \mathbf{R}_{\mathrm{y}} \mathbf{M}_{Q W}\left(\mathrm{x} 45^{\circ}+\varepsilon, \omega\right) \mathbf{M}_{O}(\gamma) \mathbf{F}(a) \boldsymbol{I}_{E}= \\
& \eta_{S} \mathbf{A}_{S}(\mathrm{y}) \mathbf{M}_{Q W}\left(\mathrm{x} 45^{\circ}+\varepsilon, \omega\right) \boldsymbol{I}_{i n}(\gamma, a, \beta, \alpha)
\end{aligned}
$$

With Eq. (D5) for the analyser part $\mathbf{A}_{S}$, Eq. (S.10.16.3) for the $\lambda / 4$ plate $\mathbf{M}_{Q W}$ with phase-shift error $\omega$, and with the input Stokes vector $\boldsymbol{I}_{\text {in }}$ from Sect. E4 we get the calibration signals $I_{S}$ in Eq. (147).

$$
\begin{aligned}
& \Delta_{Q W}=90^{\circ}+\omega \Rightarrow \\
& \frac{I_{S}}{\eta_{S} T_{S} T_{Q W} I_{i n}}=\frac{\left\langle\mathbf{M}_{S} \mathbf{R}_{\mathrm{y}}\left|\mathbf{M}_{Q W}\left(\mathrm{x} 45^{\circ}+\varepsilon, \omega\right)\right| \boldsymbol{I}_{i n}\right\rangle}{\eta_{S} T_{S} T_{Q W} I_{i n}}= \\
& =\left(\begin{array}{c}
1 \\
\mathrm{y} D_{S} \\
0 \\
0
\end{array}\left|\left(\begin{array}{cccc}
1 & 0 & 0 & 0 \\
0 & \mathrm{~s}_{2 \varepsilon}^{2}-\mathrm{c}_{2 \varepsilon}^{2} \mathrm{~s}_{\omega} & -\mathrm{s}_{2 \varepsilon} \mathrm{c}_{2 \varepsilon}\left(1+\mathrm{s}_{\omega}\right) & -\mathrm{xc}_{2 \varepsilon} \mathrm{c}_{\omega} \\
0 & -\mathrm{s}_{2 \varepsilon} \mathrm{c}_{2 \varepsilon}\left(1+\mathrm{s}_{\omega}\right) & \mathrm{c}_{2 \varepsilon}^{2}-\mathrm{s}_{2 \varepsilon}^{2} \mathrm{~s}_{\omega} & -\mathrm{Xs}_{2 \varepsilon} \mathrm{c}_{\omega} \\
0 & \mathrm{xc}_{2 \varepsilon} \mathrm{c}_{\omega} & \mathrm{Xs}_{2 \varepsilon} \mathrm{c}_{\omega} & -\mathrm{s}_{\omega}
\end{array}\right)\right| \begin{array}{l}
i_{i n} \\
q_{\text {in }} \\
u_{i n} \\
v_{i n}
\end{array}\right\rangle= \\
& =i_{i n}+\mathrm{y} D_{S}\left[\mathrm{~s}_{2 \varepsilon}\left(\mathrm{s}_{2 \varepsilon} q_{i n}-\mathrm{c}_{2 \varepsilon} u_{i n}\right)-\mathrm{c}_{2 \varepsilon} \mathrm{s}_{\omega}\left(\mathrm{c}_{2 \varepsilon} q_{i n}+\mathrm{s}_{2 \varepsilon} u_{i n}\right)-\mathrm{xc}_{2 \varepsilon} \mathrm{c}_{\omega} v_{i n}\right]
\end{aligned}
$$

Special cases: For the investigation of the effect of the phase-shift error $\omega$ we neglect the rotation error $\varepsilon$ in Eq. (147) and get the calibration signals in Eq. (148) and the gain ratios in Eq. (149).

$$
\begin{aligned}
& \varepsilon=0 \Rightarrow \\
& I_{S}=\eta_{S} T_{S} T_{Q W} I_{i n}\left[i_{i n}-\mathrm{y} D_{S}\left(\mathrm{~s}_{\omega} q_{i n}+\mathrm{xc}_{\omega} v_{i n}\right)\right] \\
& \frac{\eta^{*}}{\eta}=\frac{i_{\text {in }}-\mathrm{y} D_{R} \mathrm{~s}_{\omega} q_{i n}-\mathrm{xy} D_{R} \mathrm{c}_{\omega} v_{i n}}{i_{\text {in }}-\mathrm{y} D_{T} \mathrm{~s}_{\omega} q_{\text {in }}-\mathrm{xy} D_{T} \mathrm{c}_{\omega} v_{i n}}
\end{aligned}
$$

With a cleaned analyser, the gain ratios from Eq. (149) become Eq. (150) and for the $\Delta 90$ calibration Eq. (151), from which we can estimate the influence of a phase-shift error $\omega$.

$$
\begin{aligned}
& \text { with } \varepsilon=0, D_{T}=+1, D_{R}=-1 \Rightarrow \\
& \frac{\eta^{*}}{\eta}=\frac{i_{i n}+\mathrm{ys}_{\omega} q_{i n}+\mathrm{xyc}_{\omega} v_{i n}}{i_{\text {in }}-\mathrm{ys}_{\omega} q_{i n}-\mathrm{xyc}_{\omega} v_{i n}}
\end{aligned}
$$


$\frac{\eta_{\Delta 90}^{*}}{\eta}=\sqrt{\frac{\left(i_{i n}+\mathrm{ys}_{\omega} q_{i n}\right)^{2}-\mathrm{c}_{\omega}{ }^{2} v_{i n}{ }^{2}}{\left(i_{i n}-\mathrm{ys}_{\omega} q_{i n}\right)^{2}-\mathrm{c}_{\omega}{ }^{2} v_{i n}{ }^{2}}}$

Without phase-shift error $\omega$ in Eq. (147) but with calibrator rotation error $\varepsilon$ we get the calibration signals in Eq. (152) and the gain ratios in Eq. (153).

$\omega=0 \Rightarrow$

$I_{S}=\eta_{S} T_{S} T_{Q W} I_{i n}\left\{i_{i n}+\mathrm{y} D_{S}\left[\mathrm{~s}_{2 \varepsilon}\left(\mathrm{s}_{2 \varepsilon} q_{i n}-\mathrm{c}_{2 \varepsilon} u_{i n}\right)-\mathrm{xc}_{2 \varepsilon} v_{i n}\right]\right\}$

$\frac{\eta^{*}}{\eta}=\frac{i_{i n}+\mathrm{y} D_{R} \mathrm{~s}_{2 \varepsilon}\left(\mathrm{s}_{2 \varepsilon} q_{i n}-\mathrm{c}_{2 \varepsilon} u_{i n}\right)-\mathrm{xy} D_{R} \mathrm{c}_{2 \varepsilon} v_{i n}}{i_{i n}+\mathrm{y} D_{T} \mathrm{~s}_{2 \varepsilon}\left(\mathrm{s}_{2 \varepsilon} q_{i n}-\mathrm{c}_{2 \varepsilon} u_{i n}\right)-\mathrm{xy} D_{T} \mathrm{c}_{2 \varepsilon} v_{i n}}$

$\omega=0, D_{T}=+1, D_{R}=-1 \Rightarrow$

$\frac{\eta_{\Delta 90}^{*}}{\eta}=\sqrt{\frac{\left(i_{i n}-\mathrm{ys}_{2 \varepsilon}\left(\mathrm{s}_{2 \varepsilon} q_{i n}-\mathrm{c}_{2 \varepsilon} u_{i n}\right)\right)^{2}-\mathrm{c}_{2 \varepsilon}{ }^{2} v_{i n}{ }^{2}}{\left(i_{i n}+\mathrm{ys}_{2 \varepsilon}\left(\mathrm{s}_{2 \varepsilon} q_{i n}-\mathrm{c}_{2 \varepsilon} u_{i n}\right)\right)^{2}-\mathrm{c}_{2 \varepsilon}{ }^{2} v_{i n}{ }^{2}}}$

The terms without the $\mathrm{x}$ factor in Eq. (150) containing $\omega$ and in Eq. (153) containing $\varepsilon$ are not compensated with the $\Delta 90$ calibration in Eq. (151) and Eq. (154), even if a cleaned analyser is used. This is a disadvantage of the QWP compared to the linear polariser (see Eq. 129).

From Eq. (153) without calibrator rotation $\varepsilon$ we get the gain ratios in Eqs. (155) and (156).

$$
\begin{aligned}
& \omega=\varepsilon=0 \Rightarrow \\
& \frac{\eta^{*}}{\eta}=\frac{i_{i n}-\mathrm{xy} D_{R} v_{i n}}{i_{i n}-\operatorname{xy} D_{T} v_{i n}} \\
& \frac{\eta_{\Delta 90}^{*}}{\eta}=\sqrt{\frac{i_{i n}{ }^{2}-D_{R}{ }^{2} v_{i n}^{2}}{i_{i n}{ }^{2}-D_{T}{ }^{2} v_{i n}{ }^{2}}}
\end{aligned}
$$

With a cleaned analyser Eq. (156) becomes Eq. (157).

$\omega=\varepsilon=0, D_{T}=+1, D_{R}=-1 \Rightarrow$

$\eta_{\Delta 90}^{*}=\eta$

The advantage of the QWP calibrator is that we can retrieve from Eqs. (157) and (155) with a cleaned analyser the degree of circular polarisation $v_{i n} / i_{\text {in }}$ of the light before the polarising beam splitter according to Eq. (158). Bear in mind that $\eta^{*}$ and $\eta^{*}{ }_{\Delta 90}$ in Eq. (158) are values directly derived from measured signals. The errors due to uncertainties in $\varepsilon$ or $\omega$ can be estimated by means of equations earlier in this section. 


$$
\begin{aligned}
& \omega=\varepsilon=0, D_{T}=+1, D_{R}=-1 \Rightarrow \\
& \frac{v_{\text {in }}}{i_{\text {in }}}=\frac{1}{\mathrm{xy}} \frac{\eta^{*}-\eta_{\Delta 90}^{*}}{\eta^{*}+\eta_{\Delta 90}^{*}}
\end{aligned}
$$

\subsection{Calibration with an ideal $\lambda / 4$ plate before the receiving optics}

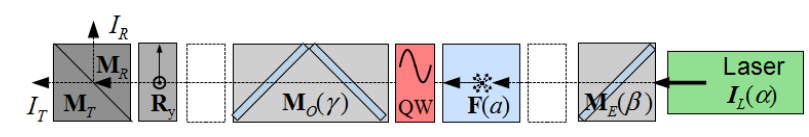

$$
\begin{aligned}
& \boldsymbol{I}_{S}=\eta_{S} \mathbf{M}_{S} \mathbf{R}(\mathrm{y}) \mathbf{M}_{O}(\gamma) \mathbf{M}_{Q W}\left(\mathrm{x} 45^{\circ}+\varepsilon\right) \mathbf{F}(a) \boldsymbol{I}_{E}(\beta, \alpha)= \\
& =\eta_{S} \mathbf{A}_{S}(\mathrm{y}, \gamma) \mathbf{M}_{Q W}\left(\mathrm{x} 45^{\circ}+\varepsilon\right) \boldsymbol{I}_{i n}(a, \beta, \alpha)
\end{aligned}
$$

With Eq. (D7) for the analyser part $\mathbf{A}_{S}$, an ideal $\lambda / 4$ plate $\mathbf{M}_{Q W}$ Eq. (S.10.17.3), and with an input Stokes vector $\boldsymbol{I}_{\text {in }}$ from Sect. E3 we get the general calibration signals $I_{S}$ in Eq. (160).

$$
\begin{aligned}
& \frac{I_{S}}{\eta_{S} T_{S} T_{O} T_{Q W} F_{11} T_{E} I_{L}}=\frac{\left\langle\mathbf{M}_{S} \mathbf{R}_{\mathrm{y}} \mathbf{M}_{O}(\gamma)\left|\mathbf{M}_{Q W}\left(\mathrm{x} 45^{\circ}+\varepsilon\right)\right| \mathbf{F}(a) \mathbf{M}_{E} \boldsymbol{I}_{L}\right\rangle}{T_{S} T_{O} T_{Q W} F_{11} T_{E} I_{L}}= \\
& \left.=\left\langle\begin{array}{c}
1+\mathrm{yc}_{2 \gamma} D_{S} D_{O} \\
\mathrm{c}_{2 \gamma} D_{O}+\mathrm{y} D_{S}\left(1-\mathrm{s}_{2 \gamma}^{2} W_{O}\right) \\
\mathrm{s}_{2 \gamma}\left(D_{O}+\mathrm{yc}_{2 \gamma} D_{S} W_{O}\right) \\
-\mathrm{ys}_{2 \gamma} D_{S} Z_{O} \mathrm{~s}_{O}
\end{array}\right| \begin{array}{cccc}
1 & 0 & 0 & 0 \\
0 & \mathrm{~s}_{2 \varepsilon}^{2} & -\mathrm{s}_{2 \varepsilon} \mathrm{c}_{2 \varepsilon} & -\mathrm{xc}_{2 \varepsilon} \\
0 & -\mathrm{s}_{2 \varepsilon} \mathrm{c}_{2 \varepsilon} & \mathrm{c}_{2 \varepsilon}^{2} & -\mathrm{xs}_{2 \varepsilon} \\
0 & \mathrm{xc}_{2 \varepsilon} & \mathrm{xs}_{2 \varepsilon} & 0
\end{array}\right)\left|\begin{array}{c}
i_{E} \\
a q_{E} \\
-a u_{E} \\
(1-2 a) v_{E}
\end{array}\right\rangle= \\
& =\left(\begin{array}{c}
1+\mathrm{yc}_{2 \gamma} D_{S} D_{O} \\
\mathrm{~s}_{2 \varepsilon} D_{O} \mathrm{~s}_{2 \varepsilon-2 \gamma}+\mathrm{y} D_{S}\left[\mathrm{~s}_{2 \varepsilon}^{2}-\mathrm{s}_{2 \varepsilon} \mathrm{s}_{2 \gamma} W_{O} \mathrm{c}_{2 \varepsilon-2 \gamma}-\mathrm{xc}_{2 \varepsilon} \mathrm{s}_{2 \gamma} Z_{O} \mathrm{~s}_{O}\right] \\
-\mathrm{c}_{2 \varepsilon} D_{O} \mathrm{~s}_{2 \varepsilon-2 \gamma}-\mathrm{y} D_{S}\left[\mathrm{~s}_{2 \varepsilon} \mathrm{c}_{2 \varepsilon}-\mathrm{c}_{2 \varepsilon} \mathrm{s}_{2 \gamma} W_{O} \mathrm{c}_{2 \varepsilon-2 \gamma}+\mathrm{xs}_{2 \varepsilon} \mathrm{s}_{2 \gamma} Z_{O} \mathrm{~s}_{O}\right] \\
-\mathrm{x}\left\{D_{O} \mathrm{c}_{2 \varepsilon-2 \gamma}+\mathrm{y} D_{S}\left[\mathrm{c}_{2 \varepsilon}+\mathrm{s}_{2 \gamma} W_{O} \mathrm{~s}_{2 \varepsilon-2 \gamma}\right]\right\} \\
a q_{E} \\
-a u_{E} \\
(1-2 a) v_{E}
\end{array}\right)
\end{aligned}
$$

Special cases: Without receiver optics rotation $\gamma$ we get from Eq. (E19) and Eq. (160) the calibration signals in Eq. (161) and the gain ratios in Eq. (162). 
$\gamma=0 \Rightarrow$

$\frac{I_{S}}{\eta_{S} T_{S} T_{O} T_{Q W} F_{11} T_{E} I_{L}}=\frac{\left\langle\mathbf{A}_{S}(\mathrm{y}, 0)\right| \mathbf{M}_{Q W}\left(\mathrm{x} 45^{\circ}+\varepsilon\right)}{T_{S} T_{O} T_{Q W}} \frac{\left|\mathbf{F}(a) \mathbf{M}_{E} \boldsymbol{I}_{L}\right\rangle}{F_{11} T_{E} I_{L}}=$

$=\left\langle\begin{array}{c|c}1+\mathrm{y} D_{S} D_{O} & i_{E} \\ \mathrm{~s}_{2 \varepsilon}^{2}\left(D_{O}+\mathrm{y} D_{S}\right) & a q_{E} \\ -\mathrm{c}_{2 \varepsilon} \mathrm{s}_{2 \varepsilon}\left(D_{O}+\mathrm{y} D_{S}\right) & -a u_{E} \\ -\mathrm{xc}_{2 \varepsilon}\left(D_{O}+\mathrm{y} D_{S}\right) & (1-2 a) v_{E}\end{array}\right\rangle=$

$=\left(1+\mathrm{y} D_{S} D_{O}\right) i_{E}+\left(D_{O}+\mathrm{y} D_{S}\right)\left[\mathrm{s}_{2 \varepsilon} a\left(\mathrm{~s}_{2 \varepsilon} q_{E}+\mathrm{c}_{2 \varepsilon} u_{E}\right)-\mathrm{xc}_{2 \varepsilon}(1-2 a) v_{E}\right]$

$\gamma=0 \Rightarrow$

$\frac{\eta^{*}}{\eta}=\frac{\left(1+\mathrm{y} D_{R} D_{O}\right) i_{E}+\left(D_{O}+\mathrm{y} D_{R}\right)\left[\mathrm{s}_{2 \varepsilon} a\left(\mathrm{~s}_{2 \varepsilon} q_{E}+\mathrm{c}_{2 \varepsilon} u_{E}\right)-\mathrm{xc}_{2 \varepsilon}(1-2 a) v_{E}\right]}{\left(1+\mathrm{y} D_{T} D_{O}\right) i_{E}+\left(D_{O}+\mathrm{y} D_{T}\right)\left[\mathrm{s}_{2 \varepsilon} a\left(\mathrm{~s}_{2 \varepsilon} q_{E}+\mathrm{c}_{2 \varepsilon} u_{E}\right)-\mathrm{xc}_{2 \varepsilon}(1-2 a) v_{E}\right]}$

Equation (162) with a cleaned PBS (Supplement Sect. S10.10) becomes Eq. (163), and without calibrator rotation $\varepsilon$ Eq. (162) becomes Eq. (164).

$$
\begin{aligned}
& \gamma=0, D_{T}=+1, D_{R}=-1 \Rightarrow \\
& \frac{\eta^{*}}{\eta}=\frac{1-\mathrm{y} D_{O}}{1+\mathrm{y} D_{O}} \frac{i_{E}-\mathrm{y}\left[\mathrm{s}_{2 \varepsilon} a\left(\mathrm{~s}_{2 \varepsilon} q_{E}+\mathrm{c}_{2 \varepsilon} u_{E}\right)-\mathrm{xc}_{2 \varepsilon}(1-2 a) v_{E}\right]}{i_{E}+\mathrm{y}\left[\mathrm{s}_{2 \varepsilon} a\left(\mathrm{~s}_{2 \varepsilon} q_{E}+\mathrm{c}_{2 \varepsilon} u_{E}\right)-\mathrm{xc}_{2 \varepsilon}(1-2 a) v_{E}\right]} \\
& \gamma=\varepsilon=0 \Rightarrow \\
& \frac{\eta^{*}}{\eta}=\frac{\left(1+\mathrm{y} D_{R} D_{O}\right) i_{E}-\mathrm{x}\left(D_{O}+\mathrm{y} D_{R}\right)(1-2 a) v_{E}}{\left(1+\mathrm{y} D_{T} D_{O}\right) i_{E}-\mathrm{x}\left(D_{O}+\mathrm{y} D_{T}\right)(1-2 a) v_{E}}
\end{aligned}
$$

With a cleaned analyser and without calibrator rotation $\varepsilon$ the gain ratios in Eq. (162) become Eq. (165) and for the $\Delta 90$ calibration Eq. (166).

$$
\begin{aligned}
& \gamma=\varepsilon=0, D_{T}=+1, D_{R}=-1 \Rightarrow \\
& \frac{\eta^{*}}{\eta}=\frac{1-\mathrm{y} D_{O}}{1+\mathrm{y} D_{O}} \frac{i_{E}+\mathrm{xy}(1-2 a) v_{E}}{i_{E}-\mathrm{xy}(1-2 a) v_{E}} \\
& \frac{\eta_{\Delta 90}^{*}}{\eta}=\frac{1-\mathrm{y} D_{O}}{1+\mathrm{y} D_{O}}
\end{aligned}
$$

Equation (165) can be rearranged with Eq. (166) to Eq. (167), from which we get the degree of circular polarisation $v_{E}$ $/ i_{E}$ of the beam behind the emitter optics in Eq. (168). The atmospheric polarisation parameter $a$ must be estimated from a standard measurement, and if we use an atmospheric range without aerosols it becomes $a \approx 1$. While $v_{i n}$ in Eq. (158) includes the mostly unknown retardation terms of the receiving optics, $v_{E}$ in Eq. (168) is free of them and hence a better estimation for the elliptical polarisation of the laser. 
$\gamma=\varepsilon=0, D_{T}=+1, D_{R}=-1 \Rightarrow$

$\frac{\eta^{*}}{\eta_{\Delta 90}^{*}}=\frac{i_{E}+\mathrm{xy}(1-2 a) v_{E}}{i_{E}-\mathrm{xy}(1-2 a) v_{E}} \Rightarrow$

$\frac{v_{E}}{i_{E}}=\frac{1}{\mathrm{xy}(1-2 a)} \frac{\eta^{*}-\eta_{\Delta 90}^{*}}{\eta^{*}+\eta_{\Delta 90}^{*}}$

\subsection{Calibration with an ideal $\lambda / 4$ plate behind the emitter optics}

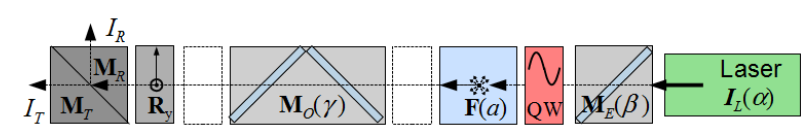

$$
\begin{aligned}
& \boldsymbol{I}_{S}=\eta_{S} \mathbf{M}_{S} \mathbf{R}(\mathrm{y}) \mathbf{M}_{O}(\gamma) \mathbf{F}(a) \mathbf{M}_{Q W}\left(\mathrm{x} 45^{\circ}+\varepsilon\right) \mathbf{M}_{E}(\beta) \boldsymbol{I}_{L}(\alpha)= \\
& =\eta_{S} \mathbf{A}_{S}(\mathrm{y}, \gamma, a) \mathbf{M}_{Q W}\left(\mathrm{x} 45^{\circ}+\varepsilon\right) \boldsymbol{I}_{i n}(\beta, \alpha)
\end{aligned}
$$

With Eq. (D13) for the analyser part $\mathbf{A}_{S}$, an ideal $\lambda / 4$ plate $\mathbf{M}_{Q W}$ Eq. (S.10.17.3), and with an input Stokes vector $\boldsymbol{I}_{\text {in }}$ from Eq. (E8) we get the general calibration signals $I_{S}$ in Eq. (170).

$$
\begin{aligned}
& \frac{I_{S}}{\eta_{S} T_{S} T_{O} F_{11} T_{Q W} T_{E} I_{L}}=\frac{\left\langle\mathbf{M}_{S} \mathbf{R}_{\mathrm{y}} \mathbf{M}_{O}(\gamma) \mathbf{F}(a)\left|\mathbf{M}_{Q W}\left(\mathrm{x} 45^{\circ}+\varepsilon\right)\right| \mathbf{M}_{E} \boldsymbol{I}_{L}\right\rangle}{T_{S} T_{O} F_{11} T_{Q W} T_{E} I_{L}}= \\
& =\left(\begin{array}{c}
a\left[\mathrm{c}_{2 \gamma} D_{O}+\mathrm{y}_{2 \gamma} D_{S}\left(1-\mathrm{s}_{2 \gamma}^{2} W_{O}\right)\right] \\
-a \mathrm{~s}_{2 \gamma}\left(D_{O}+\mathrm{yc}_{2 \gamma} D_{S} W_{O}\right) \\
-(1-2 a) \mathrm{ys}_{2 \gamma} D_{S} Z_{O} \mathrm{~s}_{O}
\end{array}\left|\left(\begin{array}{cccc}
1 & 0 & 0 & 0 \\
0 & \mathrm{~s}_{2 \varepsilon}^{2} & -\mathrm{s}_{2 \varepsilon} \mathrm{c}_{2 \varepsilon} & -\mathrm{xc}_{2 \varepsilon} \\
0 & -\mathrm{s}_{2 \varepsilon} \mathrm{c}_{2 \varepsilon} & \mathrm{c}_{2 \varepsilon}^{2} & -\mathrm{xs}_{2 \varepsilon} \\
0 & \mathrm{xc}_{2 \varepsilon} & \mathrm{xs}_{2 \varepsilon} & 0
\end{array}\right)\right| \begin{array}{l}
i_{E} \\
q_{E} \\
u_{E} \\
v_{E}
\end{array}\right\rangle
\end{aligned}
$$

Special cases: Equivalent to Sect.9.2 we get from Eq. (170) without receiver optics rotation $\gamma$ the calibration signals in Eq. (171).

with $\gamma=0 \Rightarrow$

$$
\frac{I_{S}}{\eta_{S} T_{S} T_{O} T_{Q W} F_{11} T_{E} I_{L}}=\frac{\left\langle\mathbf{A}_{S}(\mathrm{y}, 0, a)\left|\mathbf{M}_{Q W}\left(\mathrm{x} 45^{\circ}+\varepsilon\right)\right| \mathbf{M}_{E} \boldsymbol{I}_{L}\right\rangle}{T_{S} T_{O} T_{Q W} F_{11} T_{E} I_{L}}=\left(\begin{array}{c|c}
1+\mathrm{y} D_{S} D_{O} & i_{E} \\
\mathrm{~s}_{2 \varepsilon}^{2} a\left(D_{O}+\mathrm{y} D_{S}\right) & q_{E} \\
-\mathrm{c}_{2 \varepsilon} \mathrm{s}_{2 \varepsilon} a\left(D_{O}+\mathrm{y} D_{S}\right) & u_{E} \\
-\mathrm{xc}_{2 \varepsilon} a\left(D_{O}+\mathrm{y} D_{S}\right) & v_{E}
\end{array}\right)
$$

From Eq. (171) without calibrator rotation $\varepsilon$ we get the gain ratios in Eq. (172); with additionally a cleaned analyser we get Eq. (173), and with the corresponding $\Delta 90$ calibration Eq. (174). 
with $\gamma=\varepsilon=0 \Rightarrow$

$\frac{\eta^{*}}{\eta}=\frac{\left(1+\mathrm{y} D_{R} D_{O}\right) i_{E}-\mathrm{x} a\left(D_{O}+\mathrm{y} D_{R}\right) v_{E}}{\left(1+\mathrm{y} D_{T} D_{O}\right) i_{E}-\mathrm{x} a\left(D_{O}+\mathrm{y} D_{T}\right) v_{E}}$

with $\gamma=\varepsilon=0, D_{T}=+1, D_{R}=-1 \Rightarrow$

$\frac{\eta^{*}}{\eta}=\frac{1-\mathrm{y} D_{O}}{1+\mathrm{y} D_{O}} \frac{i_{E}+\mathrm{xy} a v_{E}}{i_{E}-\mathrm{xy} a v_{E}}$

$\frac{\eta_{\Delta 90}^{*}}{\eta}=\frac{1-\mathrm{y} D_{O}}{1+\mathrm{y} D_{O}}$

Equation (173) can be rearranged with Eq. (174) to Eq. (175) from which we get the degree of circular polarisation $v_{E}$ $/ i_{E}$ of the beam behind the emitter optics if the atmospheric polarisation parameter $a$ is known, e.g. when we use the lidar signals from an atmospheric range without aerosols where $a \approx 1$.

$\frac{\eta^{*}}{\eta_{\Delta 90}^{*}}=\frac{i_{E}+\operatorname{xy}^{2} v_{E}}{i_{E}-\operatorname{xy} a v_{E}} \Rightarrow \frac{v_{E}}{i_{E}}=\frac{1}{\operatorname{xy} a} \frac{\eta^{*}-\eta_{\Delta 90}^{*}}{\eta^{*}+\eta_{\Delta 90}^{*}}$

\section{Calibration with a circular polariser (CP)}

The use of a circular polariser seems to be ideal for the calibration, but the uncertainties of a real circular polariser are usually not provided by manufacturers and might be difficult to determine. A real CP is mostly a combination of a linear

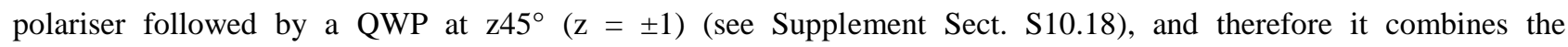
uncertainties of both (see Sects. 8 and 9). Before the results of a circularly polarising calibrator can be trusted, the diattenuation of the linear polariser and the phase-shift uncertainties should be determined and the error assessment performed using the general Eq. (C10) for the calibration signals. If we consider all possible error terms, the Müller matrix for a real CP becomes too complex for this investigation; therefore, we assume a circular polariser with phaseshift error $\omega$ but with an ideal linear polariser from Eq. (S.10.18.4) in the following in order to show the possibilities of this calibrator.

\subsection{Calibration with a circular polariser before the polarising beam splitter}

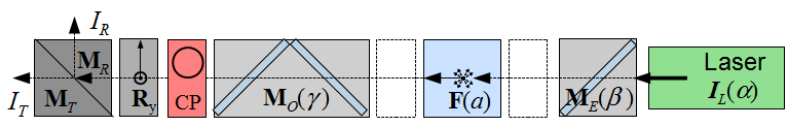

$$
\begin{aligned}
& \boldsymbol{I}_{S}=\eta_{S} \mathbf{M}_{S} \mathbf{R}_{\mathrm{y}} \mathbf{M}_{C P}\left(\mathrm{z}, \mathrm{X} 45^{\circ}+\varepsilon, \omega\right) \mathbf{M}_{o}(\gamma) \mathbf{F}(a) \mathbf{M}_{E}(\beta) \boldsymbol{I}_{L}(\alpha)= \\
& =\eta_{S} \mathbf{A}_{S}(\mathrm{y}) \mathbf{M}_{C P}\left(\mathrm{z}, \mathrm{x} 45^{\circ}+\varepsilon, \omega\right) \boldsymbol{I}_{i n}(\gamma, a, \beta, \alpha)
\end{aligned}
$$


With $\mathbf{A}_{S}$ from Eq. (D5), the circularly polarising calibrator $\mathbf{M}_{C P}$ with retardation error $\omega$ from Eq. (S.10.18.4), and the input Stokes vectors $\boldsymbol{I}_{\text {in }}$ from Sect. E4 we get Eq. (177) for the calibration signals $I_{S}$. As the last term of Eq. (177) is independent of $D_{S}$, it cancels out in the gain ratios in Eq. (178), which is therefore independent of the input Stokes vector, but still includes $\varepsilon$ and $\omega$ terms.

$$
\begin{aligned}
& \frac{I_{S}}{\eta_{S} T_{S} T_{C P} I_{\text {in }}}=\frac{\left\langle\mathbf{M}_{S} \mathbf{R}_{\mathrm{y}}\left|\mathbf{M}_{C P}\left(\mathrm{z}, \mathrm{x} 45^{\circ}+\varepsilon, \omega\right)\right| \boldsymbol{I}_{\text {in }}\right\rangle}{T_{S} T_{C P} I_{i n}}=
\end{aligned}
$$

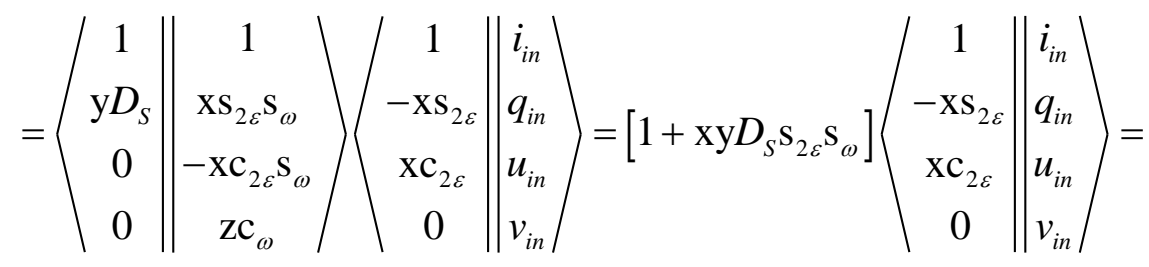

$$
\begin{aligned}
& =\left(1+\mathrm{xy} D_{S} \mathrm{~s}_{2 \varepsilon} \mathrm{s}_{\omega}\right)\left[i_{\text {in }}-\mathrm{x}\left(\mathrm{s}_{2 \varepsilon} q_{\text {in }}-\mathrm{c}_{2 \varepsilon} u_{\text {in }}\right)\right] \\
& \frac{\eta^{*}}{\eta}=\frac{1+\mathrm{xy} D_{R} \mathrm{~s}_{2 \varepsilon} \mathrm{s}_{\omega}}{1+\mathrm{xy}_{T} \mathrm{~s}_{2 \varepsilon} \mathrm{s}_{\omega}}
\end{aligned}
$$

If $\omega$ is zero, we have an ideal circular polariser with which we get the gain ratio independently of $\varepsilon$, and if $\varepsilon$ is zero $\omega$ does not matter (Eq. 179).

$$
\begin{aligned}
& \omega=0 \vee \varepsilon=0 \Rightarrow \\
& \frac{\eta^{*}}{\eta}=1
\end{aligned}
$$

With a cleaned analyser from Eq. (178) we get Eqs. (180) and (181), which show that the deviations of the gain ratios are fully compensated by the $\Delta 90$ calibration. $\omega$ can be determined by means of the successive approximation in Sect. 11, Eq. (198)ff.

$$
\begin{aligned}
& D_{T}=+1, D_{R}=-1 \Rightarrow \\
& \frac{\eta^{*}}{\eta}=\frac{1-\operatorname{xys}_{2 \varepsilon} \mathrm{s}_{\omega}}{1+\operatorname{xys}_{2 \varepsilon} \mathrm{s}_{\omega}} \\
& \frac{\eta_{\Delta 90}^{*}}{\eta}=1
\end{aligned}
$$

\subsection{Calibration with a circular polariser before the receiving optics}

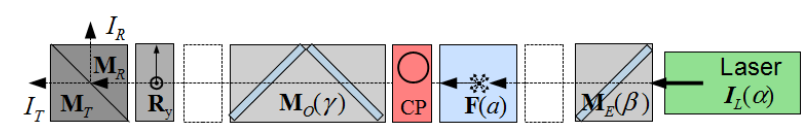


$\boldsymbol{I}_{S}=\eta_{S} \mathbf{M}_{S} \mathbf{R}_{\mathrm{y}} \mathbf{M}_{o}(\gamma) \mathbf{M}_{C P}\left(\mathrm{z}, \mathrm{x} 45^{\circ}+\varepsilon, \omega\right) \mathbf{F}(a) \mathbf{M}_{E}(\beta) \boldsymbol{I}_{L}(\alpha)=$

$=\eta_{S} \mathbf{A}_{S}(\mathrm{y}, \gamma) \mathbf{M}_{C P}\left(\mathrm{z}, \mathrm{x} 45^{\circ}+\varepsilon, \omega\right) \boldsymbol{I}_{\text {in }}(a, \beta, \alpha)$

With $\mathbf{A}_{S}$ from Sect. D2, $\mathbf{M}_{C P}$ with retardation error $\omega$ from Eq. (S.10.18.4), and $\boldsymbol{I}_{\text {in }}$ from Sect. E3 we get Eq. (183) for the calibration signals $I_{S}$.

$$
\begin{aligned}
& \frac{I_{S}}{\eta_{S} T_{S} T_{C P} T_{O} F_{11} T_{E} I_{L}}=\frac{\left\langle\mathbf{M}_{S} \mathbf{R}_{\mathrm{y}} \mathbf{M}_{O}(\gamma)\left|\mathbf{M}_{C P}\left(\mathrm{z}, \mathbf{x} 45^{\circ}+\varepsilon, \omega\right)\right| \mathbf{F}(a) \mathbf{M}_{E} \boldsymbol{I}_{L}\right\rangle}{T_{S} T_{O} T_{C P} F_{11} T_{E} I_{L}}= \\
& \left.=\left\{\begin{array}{c}
1+\mathrm{yc}_{2 \gamma} D_{S} D_{O} \\
\mathrm{c}_{2 \gamma} D_{O}+\mathrm{y}_{S}\left(1-\mathrm{s}_{2 \gamma}^{2} W_{O}\right) \\
\mathrm{s}_{2 \gamma}\left(D_{O}+\mathrm{yc}_{2 \gamma} D_{S} W_{O}\right) \\
-\mathrm{ys}_{2 \gamma} D_{S} Z_{O} \mathrm{~s}_{O}
\end{array} \mid \begin{array}{c}
1 \\
\mathrm{xs}_{2 \varepsilon} \mathrm{s}_{\omega} \\
-\mathrm{xc}_{2 \varepsilon} \mathrm{s}_{\omega} \\
\mathrm{zc}_{\omega}
\end{array}\right)\left|\begin{array}{c}
1 \\
-\mathrm{xs}_{2 \varepsilon} \\
\mathrm{xc}_{2 \varepsilon} \\
0
\end{array}\right| \begin{array}{c}
i_{E} \\
a q_{E} \\
-a u_{E} \\
(1-2 a) v_{E}
\end{array}\right)= \\
& =\left\{\begin{array}{c}
1+\mathrm{y}_{S}\left(\mathrm{c}_{2 \gamma} D_{O}-\mathrm{s}_{2 \gamma} Z_{O} \mathrm{~s}_{O} \mathrm{zc}_{\omega}\right)+ \\
+\mathrm{xs}_{\omega}\left[D_{O} \mathrm{~s}_{2 \varepsilon-2 \gamma}+\mathrm{y}_{S}\left(\mathrm{~s}_{2 \varepsilon}-\mathrm{s}_{2 \gamma} W_{O} \mathrm{c}_{2 \varepsilon-2 \gamma}\right)\right]
\end{array}\right\}\left[\begin{array}{c}
\left.i_{E}-\mathrm{x} a\left(\mathrm{~s}_{2 \varepsilon} q_{E}+\mathrm{c}_{2 \varepsilon} u_{E}\right)\right]
\end{array}\right.
\end{aligned}
$$

As the last term of Eq. (183) is independent of $D_{S}$, it cancels out in the gain ratio. However, as long as the receiver optics rotation $\gamma$ does not vanish, the gain ratios include deviations which do not cancel out with the $\Delta 90$ calibration, even if we used a cleaned analyser (Eq. 184) and additionally an ideal circular polariser (Eq. 185), or without calibrator error $\varepsilon$ (Eq. 186).

$$
\begin{aligned}
& D_{T}=+1, D_{R}=-1 \Rightarrow \\
& \frac{\eta^{*}}{\eta}=\frac{1-\mathrm{y}\left(\mathrm{c}_{2 \gamma} D_{O}-\mathrm{s}_{2 \gamma} Z_{O} \mathrm{~s}_{O} \mathrm{zc}_{\omega}\right)+\mathrm{xs}_{\omega}\left[D_{O} \mathrm{~s}_{2 \varepsilon-2 \gamma}-\mathrm{y}\left(\mathrm{s}_{2 \varepsilon}-\mathrm{s}_{2 \gamma} W_{O} \mathrm{c}_{2 \varepsilon-2 \gamma}\right)\right]}{1+\mathrm{y}\left(\mathrm{c}_{2 \gamma} D_{O}-\mathrm{s}_{2 \gamma} Z_{O} \mathrm{~s}_{O} \mathrm{zc}_{\omega}\right)+\mathrm{xs}_{\omega}\left[D_{O} \mathrm{~s}_{2 \varepsilon-2 \gamma}+\mathrm{y}\left(\mathrm{s}_{2 \varepsilon}-\mathrm{s}_{2 \gamma} W_{O} \mathrm{c}_{2 \varepsilon-2 \gamma}\right)\right]} \\
& D_{T}=+1, D_{R}=-1, \omega=0 \Rightarrow \\
& \frac{\eta^{*}}{\eta}=\frac{1-\mathrm{y}\left(\mathrm{c}_{2 \gamma} D_{O}-\mathrm{s}_{2 \gamma} Z_{O} \mathrm{~s}_{O}\right)}{1+\mathrm{y}\left(\mathrm{c}_{2 \gamma} D_{O}-\mathrm{s}_{2 \gamma} Z_{O} \mathrm{~s}_{O}\right)} \\
& \frac{\eta_{T}^{*}}{\eta}=+1, D_{R}=-1, \varepsilon=0 \Rightarrow \\
& 1+\mathrm{y}\left(\mathrm{c}_{2 \gamma} D_{O}-\mathrm{s}_{2 \gamma} Z_{O} \mathrm{~s}_{O} \mathrm{zc}_{\omega}\right)-\mathrm{xs}_{\omega} \mathrm{s}_{2 \gamma}\left[D_{O}-\mathrm{s}_{2 \gamma} Z_{O} \mathrm{~s}_{O} \mathrm{zc}_{\omega}\right)-\mathrm{xs}_{\omega} \mathrm{s}_{2 \gamma}\left[D_{O}+\mathrm{y} W_{O} \mathrm{c}_{2 \gamma}\right]
\end{aligned}
$$

From Eq. (183) without receiver optics rotation $\gamma$ we get Eq. (187), and with additionally a cleaned analyser Eqs. (188) and (189) are the same as in the previous sections but with the prefactor of Eq. (189). 
$\gamma=0 \Rightarrow \quad \frac{\eta^{*}}{\eta}=\frac{1+\mathrm{y} D_{R} D_{O}+\mathrm{xs}_{\omega} \mathrm{s}_{2 \varepsilon}\left(D_{O}+\mathrm{y} D_{R}\right)}{1+\mathrm{y} D_{T} D_{O}+\mathrm{xs}_{\omega} \mathrm{s}_{2 \varepsilon}\left(D_{O}+\mathrm{y} D_{T}\right)}$

$\gamma=0, D_{T}=+1, D_{R}=-1 \Rightarrow \quad \frac{\eta^{*}}{\eta}=\frac{1-\mathrm{y} D_{O}}{1+\mathrm{y} D_{O}} \frac{1-\mathrm{xys}_{\omega} \mathrm{s}_{2 \varepsilon}}{1+\mathrm{xys}_{\omega} \mathrm{s}_{2 \varepsilon}}$

$\left[\gamma=0, D_{T}=+1, D_{R}=-1\right] \wedge[\omega=0 \vee \varepsilon=0] \Rightarrow \frac{\eta^{*}}{\eta}=\frac{1-\mathrm{y} D_{O}}{1+\mathrm{y} D_{O}}$

\subsection{Calibration with a circular polariser behind the emitter optics}

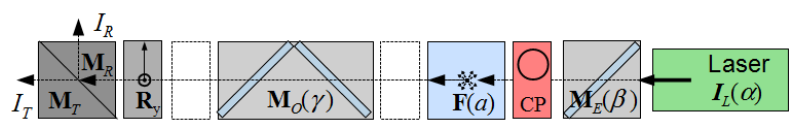

$$
\begin{aligned}
& \boldsymbol{I}_{S}=\eta_{S} \mathbf{M}_{S} \mathbf{R}_{\mathrm{y}} \mathbf{M}_{o}(\gamma) \mathbf{F}(a) \mathbf{M}_{C P}\left(\mathrm{z}, \mathrm{x} 45^{\circ}+\varepsilon\right) \mathbf{M}_{E}(\beta) \boldsymbol{I}_{L}(\alpha)= \\
& =\eta_{S} \mathbf{A}_{S}(\mathrm{y}, \gamma, a) \mathbf{M}_{C P}\left(\mathrm{z}, \mathrm{x} 45^{\circ}+\varepsilon\right) \boldsymbol{I}_{E}(\beta, \alpha)
\end{aligned}
$$

With $\mathbf{A}_{S}$ from 0, $\mathbf{M}_{C P}$ with retardation error $\omega$ from Eq. (S.10.18.4), and $\boldsymbol{I}_{i n}$ from Sect. E2 we get Eq. (191) for the calibration signals $I_{S}$, which differs from Eq. (183) in the last section just by the prefactors depending on the atmospheric polarisation parameter $a$. The same holds for the gain ratios derived with a cleaned analyser in Eq. (192) compared to Eq. (184) and all the subsequent derivations there.

$$
\begin{aligned}
& \frac{I_{S}}{\eta_{S} T_{S} T_{O} F_{11} T_{C P} T_{E} I_{L}}=\frac{\left\langle\mathbf{M}_{S} \mathbf{R}_{\mathrm{y}} \mathbf{M}_{O}(\gamma) \mathbf{F}(a)\left|\mathbf{M}_{C P}\left(\mathrm{z}, \mathrm{X} 45^{\circ}+\varepsilon, \omega\right)\right| \mathbf{M}_{E} \boldsymbol{I}_{L}\right\rangle}{T_{S} T_{O} F_{11} T_{C P} T_{E} I_{L}}= \\
& =\left\langle\begin{array}{c}
a\left[\mathrm{c}_{2 \gamma} D_{O}+\mathrm{yc}_{2 \gamma} D_{S}\left(1-\mathrm{s}_{2 \gamma}^{2} W_{O}\right)\right] \\
-a \mathrm{~s}_{2 \gamma}\left(D_{O}+\mathrm{yc}_{2 \gamma} D_{S} W_{O}\right) \\
-(1-2 a) \mathrm{ys}_{2 \gamma} D_{S} Z_{O} \mathrm{~s}_{O}
\end{array}|| \begin{array}{c}
1 \\
\mathrm{xs}_{2 \varepsilon} \mathrm{s}_{\omega} \\
-\mathrm{xc}_{2 \varepsilon} \mathrm{s}_{\omega} \\
\mathrm{zc}_{\omega}
\end{array}\right)\left\langle\begin{array}{c}
1 \\
-\mathrm{xs}_{2 \varepsilon} \\
\mathrm{xc}_{2 \varepsilon} \\
0
\end{array}\right. \\
& =\left\{\begin{array}{l}
1+\mathrm{y} D_{S}\left(\mathrm{c}_{2 \gamma} D_{O}-(1-2 a) \mathrm{s}_{2 \gamma} Z_{O} \mathrm{~s}_{O} \mathrm{zc}_{\omega}\right)+ \\
+\mathrm{x} \mathrm{s}_{\omega}\left[D_{O} \mathrm{~s}_{2 \varepsilon-2 \gamma}+\mathrm{y} D_{S}\left(\mathrm{~s}_{2 \varepsilon}-\mathrm{s}_{2 \gamma} W_{O} \mathrm{c}_{2 \varepsilon-2 \gamma}\right)\right]
\end{array}\right\}\left[i_{E}-\mathrm{x}\left(\mathrm{s}_{2 \varepsilon} q_{E}+\mathrm{c}_{2 \varepsilon} u_{E}\right)\right] \\
& D_{T}=+1, D_{R}=-1 \Rightarrow \\
& \frac{\eta^{*}}{\eta}=\frac{1-\mathrm{y}\left(\mathrm{c}_{2 \gamma} D_{O}-\mathrm{s}_{2 \gamma}(1-2 a) Z_{O} \mathrm{~s}_{O} \mathrm{zc}_{\omega}\right)+\mathrm{x} a \mathrm{~s}_{\omega}\left[D_{O} \mathrm{~s}_{2 \varepsilon-2 \gamma}-\mathrm{y}\left(\mathrm{s}_{2 \varepsilon}-\mathrm{s}_{2 \gamma} W_{O} \mathrm{c}_{2 \varepsilon-2 \gamma}\right)\right]}{1+\mathrm{y}\left(\mathrm{c}_{2 \gamma} D_{O}-\mathrm{s}_{2 \gamma}(1-2 a) Z_{O} \mathrm{~s}_{O} \mathrm{zc}_{\omega}\right)+\mathrm{x} a \mathrm{~s}_{\omega}\left[D_{O} \mathrm{~s}_{2 \varepsilon-2 \gamma}+\mathrm{y}\left(\mathrm{s}_{2 \varepsilon}-\mathrm{s}_{2 \gamma} W_{O} \mathrm{c}_{2 \varepsilon-2 \gamma}\right)\right]}
\end{aligned}
$$




\section{Determination of the calibrator rotation $\varepsilon$}

The calibration measurements can be used to determine and consequentially correct the calibrator rotation $\varepsilon$, which is especially important for the rotation calibrator (Sect. 7) because here the rotation error $\varepsilon$ is also present in the standard measurements and has to be corrected, either mechanically before the measurements or analytically after the measurements. If the $\pm 45^{\circ}$ calibration measurements can be described or approximated by Eq. (193) with $f(\mathrm{y}, \ldots)$ being a function of any parameter but not of $\mathrm{x}$ and $\varepsilon$, it is possible to estimate the calibrator rotation $\varepsilon$ by means of the relative difference of the $\pm 45^{\circ}$ gain ratios as in Eq. (194) and using the tangent half-angle substitution (Supplement Sect. S12.1) to achieve $\varepsilon$ from Eq. (195). Note that $\eta$ is assumed to be unknown.

$$
\frac{\eta^{*}}{\eta}=f(\mathrm{y}, \ldots) \frac{1+\mathrm{xs}_{2 \varepsilon}}{1-\mathrm{xs}_{2 \varepsilon}}
$$

$Y(\varepsilon) \equiv \frac{\eta^{*}\left(\mathrm{y},+45^{\circ}+\varepsilon\right)-\eta^{*}\left(\mathrm{y},-45^{\circ}+\varepsilon\right)}{\eta^{*}\left(\mathrm{y},+45^{\circ}+\varepsilon\right)+\eta^{*}\left(\mathrm{y},-45^{\circ}+\varepsilon\right)}=\frac{\frac{1+\mathrm{s}_{2 \varepsilon}}{1-\mathrm{s}_{2 \varepsilon}}-\frac{1-\mathrm{s}_{2 \varepsilon}}{1+\mathrm{s}_{2 \varepsilon}}}{\frac{1+\mathrm{s}_{2 \varepsilon}}{1-\mathrm{s}_{2 \varepsilon}}+\frac{\mathrm{s}_{2 \varepsilon}}{1+\mathrm{s}_{2 \varepsilon}}}=\frac{2 \mathrm{~s}_{2 \varepsilon}}{1+\mathrm{s}_{2 \varepsilon}^{2}}$

$\varepsilon(Y)=0.5 * \arcsin [\tan (0.5 * \arcsin [Y])]$

With the assumption $\sin (2 \varepsilon)<<1$ we get a good approximation for $\varepsilon$ in the simple Eq. (196), which deviates by about $5 \%$ at $\varepsilon \approx 6^{\circ}$ and $Y(\varepsilon) \approx 0.4$.

$\mathrm{s}_{2 \varepsilon} \ll 1 \Rightarrow Y(\varepsilon) \approx 2 \mathrm{~s}_{2 \varepsilon} \Rightarrow \quad \varepsilon \approx 0.25 * Y$

Equation (193) is applicable in Eqs. (130) and (137) for the linear polariser calibrator, and it is a good approximation for Eq. (144) if the atmospheric polarisation parameter $a \approx 1$. For the rotation calibration before the receiving optics (Sect. 7.2, Eq. 117), we have to assume that $a \approx 1$ and additionally that the laser beam behind the emitter optics is horizontally linearly polarised. Eq. (117) can then be approximated by Eq. (197).

with $\gamma=0, D_{T}=+1, D_{R}=-1, i_{E}=q_{E}=1, u_{E}=v_{E}=0, a \approx 1 \Rightarrow$

$\frac{\eta^{*}}{\eta} \approx \frac{1-\mathrm{y}_{O}}{1+\mathrm{y} D_{O}} \frac{1+\mathrm{xs}_{2 \varepsilon}}{1-\mathrm{xs}_{2 \varepsilon}}$

If instead of Eq. (193) we have a form such as Eq. (198) (see Supplement Sect. S12.1), we get Eqs. (199) and (200). If $\varepsilon$ is known, Eq. (200) can be solved for $K$, which yields Eq. (201).

$\frac{\eta^{*}}{\eta}=f(\mathrm{y}, \ldots) \frac{1+K \mathrm{xs}_{2 \varepsilon}}{1-K \mathrm{xs}_{2 \varepsilon}}$ with $\quad K \leq 1$ 


$$
\begin{aligned}
& Y(\varepsilon, K) \equiv \frac{\eta^{*}\left(\mathrm{y},+45^{\circ}+\varepsilon, K\right)-\eta^{*}\left(\mathrm{y},-45^{\circ}+\varepsilon, K\right)}{\eta^{*}\left(\mathrm{y},+45^{\circ}+\varepsilon, K\right)+\eta^{*}\left(\mathrm{y},-45^{\circ}+\varepsilon, K\right)}=\frac{2 K \mathrm{~s}_{2 \varepsilon}}{1+K^{2} \mathrm{~s}_{2 \varepsilon}^{2}} \\
& \varepsilon=\frac{1}{2} \arcsin \left[\frac{1}{K} \tan \left(\frac{\arcsin [Y(\varepsilon, K)]}{2}\right)\right] \\
& K=\left[\frac{1}{\sin 2 \varepsilon} \tan \left(\frac{\arcsin [Y(\varepsilon, K)]}{2}\right)\right]
\end{aligned}
$$

If the true $\varepsilon$ and $K$ are unknown, we can retrieve them by successive approximation. With $K<1$ we find as a first approximation $\varepsilon_{l}$ from Eq. (202) and make the next calibration measurement after adjusting the calibrator rotation by $-\varepsilon_{1}$, which results in the actual position $\left(\varepsilon-\varepsilon_{l}\right)$ and the corresponding Eq. (203).

$\varepsilon_{1}=\frac{1}{2} \arcsin \left[\tan \left(\frac{\arcsin [Y(\varepsilon, K)]}{2}\right)\right]<\varepsilon$

$Y\left(\varepsilon-\varepsilon_{1}, K\right)=\frac{2 K \mathrm{~s}_{2\left(\varepsilon-\varepsilon_{1}\right)}}{1+K^{2} \mathrm{~s}_{2\left(\varepsilon-\varepsilon_{1}\right)}^{2}}$

Using the calibration measurements at the two positions $\varepsilon$ and $\left(\varepsilon-\varepsilon_{l}\right)$ with Eqs. (199) and (203), we get an estimation of the true $\varepsilon$ with Eq. (205) derived from the ratio in Eq. (204).

$$
\begin{aligned}
& \frac{Y\left(\varepsilon-\varepsilon_{1}, K\right)}{Y(\varepsilon, K)}=\frac{\left(1+K^{2} \mathrm{~s}_{2 \varepsilon}^{2}\right) 2 K \mathrm{~s}_{2\left(\varepsilon-\varepsilon_{1}\right)}}{\left(1+K^{2} \mathrm{~s}_{2\left(\varepsilon-\varepsilon_{1}\right)}^{2}\right) 2 K \mathrm{~s}_{2 \varepsilon}} \approx \frac{\mathrm{s}_{2\left(\varepsilon-\varepsilon_{1}\right)}}{\mathrm{s}_{2 \varepsilon}} \approx \frac{\left(\varepsilon-\varepsilon_{1}\right)}{\varepsilon}=1-\frac{\varepsilon_{1}}{\varepsilon} \\
& \varepsilon \approx \frac{Y(\varepsilon, K)}{Y(\varepsilon, K)-Y\left(\varepsilon-\varepsilon_{1}, K\right)} \varepsilon_{1}
\end{aligned}
$$

Finally, with known $\varepsilon$, we can use Eq. (201) to estimate $K$.

\section{Determination of the rotation $\alpha$ of the plane of polarisation of the emitted laser beam.}

The orientation of the plane of polarisation of the laser beam is in general specified by manufacturers just as vertical or horizontal, without specifying the reference and the accuracy. Furthermore, the assembly of the laser with the telescope and the receiver optics in a lidar system can often not be done with similar accuracy as the assembly of the optical elements in the receiver optics, and the necessary alignment mechanisms for the tilt between the laser and telescope axes additionally introduce variability and uncertainty. On top of that, the adjustments may change after every laser maintenance. Therefore it is desirable to determine the laser rotation once in a while. 
Using the calibrator equations for the calibrator before the receiver optics from 0 with an analyser without receiver optics rotation $(\gamma=0$; Eq. D8), i.e.

$$
\begin{aligned}
& \gamma=0^{\circ} \Rightarrow\left\langle\mathbf{A}_{S}\right|\left(\mathrm{y}, 0^{\circ}\right)=\left\langle\mathbf{M}_{S} \mathbf{R}_{\mathrm{y}}\right| \mathbf{M}_{O}\left(0^{\circ}\right)=T_{O} T_{S}\left\langle 1+\mathrm{y} D_{S} D_{O} \quad D_{O}+\mathrm{y} D_{S} \quad 0 \quad 0\right|= \\
& =\left\langle\mathbf{M}_{S \mathrm{y} O}\left(0^{\circ}\right)\right|=T_{\text {Sy }}\left\langle\begin{array}{llll}
1 & D_{\text {Sy } O} & 0 & 0
\end{array}\right| \\
& \Rightarrow A_{S}^{3}=A_{S}^{4}=0
\end{aligned}
$$

with elliptically polarised emitted laser light as Eq. (E.25),

$$
\frac{\boldsymbol{I}_{\text {in }}(a, b, \alpha)}{I_{\text {in }}}=\frac{\mathbf{F}(a) \boldsymbol{I}_{E}}{F_{11} T_{E} I_{L}}=\left|\begin{array}{llll}
1 & a b c_{2 \alpha} & -a b s_{2 \alpha} & (1-2 a) \sqrt{1-b^{2}}
\end{array}\right\rangle
$$

and with ideal calibrators, we get the signals for the four ideal calibrator types in Eqs. (206) to (209).

$$
\begin{aligned}
& \frac{I_{S}}{\eta_{S} I_{i n}}=\frac{\left\langle\mathbf{A}_{S}\left|\mathbf{M}_{r o t}\left(\mathrm{x} 45^{\circ}+\varepsilon, \mathrm{h}\right)\right| \boldsymbol{I}_{i n}\right\rangle}{I_{i n}}= \\
& =A_{S}^{1} i_{i n}+A_{S}^{4} \mathrm{~h} v_{i n}-\mathrm{x}\left[\left(\mathrm{s}_{2 \varepsilon} A_{S}^{2}-\mathrm{c}_{2 \varepsilon} A_{S}^{3}\right) q_{i n}+\left(\mathrm{c}_{2 \varepsilon} A_{S}^{2}+\mathrm{s}_{2 \varepsilon} A_{S}^{3}\right) \mathrm{h} u_{i n}\right]= \\
& =T_{\text {Syo }}\left(1-\mathrm{x} a b D_{S y O} \mathrm{~s}_{2 \varepsilon-\mathrm{h} 2 \alpha}\right) \\
& D_{P}=1 \Rightarrow \\
& \frac{I_{S}}{\eta_{S} T_{P} I_{i n}}=\frac{\left\langle\mathbf{A}_{S}\left|\mathbf{M}_{P}\left(\mathrm{x} 45^{\circ}+\varepsilon\right)\right| \boldsymbol{I}_{i n}\right\rangle}{T_{P} I_{i n}}=\left[A_{S}^{1}+\mathrm{x}\left(\mathrm{c}_{2 \varepsilon} A_{S}^{3}-\mathrm{s}_{2 \varepsilon} A_{S}^{2}\right)\right]\left[i_{i n}+\mathrm{x}\left(\mathrm{c}_{2 \varepsilon} u_{i n}-\mathrm{s}_{2 \varepsilon} q_{i n}\right)\right]= \\
& =T_{S y O}\left(1-\mathrm{xs}_{2 \varepsilon} D_{S y O}\right)\left(1-\mathrm{x} a b \mathrm{~s}_{2 \alpha+2 \varepsilon}\right)=T_{S y O}\left[1+a b D_{S y O} \mathrm{~s}_{2 \varepsilon} \mathrm{s}_{2 \alpha+2 \varepsilon}-\mathrm{x}\left(\mathrm{s}_{2 \varepsilon} D_{S y O}+a b \mathrm{~s}_{2 \alpha+2 \varepsilon}\right)\right] \\
& \\
& \omega=0 \Rightarrow \\
& \frac{I_{S}}{\eta_{S} T_{Q W} I_{i n}}=\frac{\left\langle\mathbf{A}_{S}\left|\mathbf{M}_{Q W}\left(\mathrm{x} 45^{\circ}+\varepsilon, 0\right)\right| \boldsymbol{I}_{i n}\right\rangle}{T_{Q W} I_{i n}}= \\
& =A_{S}^{1} i_{i n}-\left(\mathrm{s}_{2 \varepsilon} A_{S}^{2}-\mathrm{c}_{2 \varepsilon} A_{S}^{3}\right)\left(\mathrm{s}_{2 \varepsilon} q_{i n}-\mathrm{c}_{2 \varepsilon} u_{i n}\right)-\mathrm{x}\left[A_{S}^{4}\left(\mathrm{c}_{2 \varepsilon} q_{i n}+\mathrm{s}_{2 \varepsilon} u_{i n}\right)+\left(\mathrm{c}_{2 \varepsilon} A_{S}^{2}+\mathrm{s}_{2 \varepsilon} A_{S}^{3}\right) v_{i n}\right]= \\
& =T_{S y O}\left[1-a b D_{S y O} \mathrm{~s}_{2 \varepsilon} \mathrm{s}_{2 \varepsilon+2 \alpha}+\mathrm{x} D_{S y O} \mathrm{c}_{2 \varepsilon}(1-2 a) \sqrt{1-b^{2}}\right] \\
& T_{C P} I_{i n} \\
& \frac{I_{S}}{\eta_{S} T_{C P} I_{i n}}=\frac{\left\langle\mathbf{A}_{S}\left|\mathbf{M}_{C P}\left(\mathrm{z}, \mathrm{x} 45^{\circ}+\varepsilon\right)\right| \boldsymbol{I}_{i n}\right\rangle}{I_{S y O}}=\left(1+A_{S}^{1}+\mathrm{z} A_{S}^{4}\right)\left(i_{i n}-\mathrm{x}\left(\mathrm{c}_{2 \varepsilon} u_{i n}-\mathrm{s}_{2 \varepsilon} q_{i n}\right)\right)= \\
& \left.=T_{2 \alpha+2 \varepsilon}\right) \\
& =1 \Rightarrow
\end{aligned}
$$


Equations (206) and (209) are of the type of Eq. (193); therefore, the solutions described in Sect. 11 can be applied, but only to determine $\varepsilon \pm \alpha$. In order to determine $\alpha$ alone, $\varepsilon$ must be known, or a series of measurements with variable $\varepsilon$ are fitted to the gain ratios $\eta^{*}$ formulated with one of the Eqs. (206) to (209), as explained by Alvarez et al. (2006).

Furthermore, for the case of the linear polariser calibrator (Eq. 207), an unpolarised light source (i.e. $i_{i n}=1$ and $q_{i n}=u_{i n}$ $=v_{i n}=0$ ) before the receiver optics/telescope gives Eq. (210) from Eq. (207), which is of the type of Eq. (193), and with a cleaned analyser $D_{S y O}= \pm 1$.

$D_{P}=1, \quad i_{\text {in }}=1, \quad q_{\text {in }}=u_{\text {in }}=v_{\text {in }}=0 \Rightarrow$

$\frac{I_{S}}{\eta_{S} T_{P} I_{i n}}=\frac{\left\langle\mathbf{A}_{S}\left|\mathbf{M}_{P}\left(\mathrm{x} 45^{\circ}+\varepsilon\right)\right| \boldsymbol{I}_{i n}\right\rangle}{T_{P} I_{\text {in }}}=T_{S \mathrm{y} O}\left(1-\mathrm{xs}_{2 \varepsilon} D_{S \mathrm{~S} O}\right)$

\section{Assumptions and constraints of the model}

1. The correction of the standard signals (Sect. 4) and of the calibration factor (Sect. 5ff.) is only applicable in scattering ranges without aerosol or with randomly oriented, non-spherical particles with rotation and reflection symmetry as described in Sect. 2.1, and not for clouds with oriented particles as in cirrus and rain clouds (Kaul et al., 2004; Hayman et al., 2014; Volkov et al., 2015). However, the scattering volume for the calibration measurements can be chosen to avoid oriented particles in the calibration range, and then the calibration corrections in Sects. 5 to 10 can be applied for the retrieval of the calibration factor $\eta=\left(\eta_{R} T_{R}\right) /\left(\eta_{T} T_{T}\right)$, which itself is general for the considered types of lidar setups in Fig. 1.

2. We assume that the extinction in the range between the lidar and the scattering volume is polarisationindependent and that signal contributions due to multiple scattering can be neglected.

3. We assume that the atmospheric depolarisation in the calibration range does not change between the two measurements of the $\Delta 90$ calibrations. This can be verified by comparison of standard measurements before and after and possibly between the two calibration measurements.

4. Not considered are range-dependent effects as the overlap function and the range-dependent transmission and polarisation of interference filters and dichroic beam splitters, which is caused by the range-dependent incident angles on the optics.

5. We assume that the optical elements of the lidar do not depolarize. Such depolarization can be caused by optical elements inside the emitter and receiver optics, which are not well aligned with their optical axes, and by variable retardation or diattenuation over the aperture of optical elements, for example due to crystalline (e.g. $\mathrm{CaF}_{2}$ and $\mathrm{MgF}_{2}$ lenses) or stress birefringence. The latter can be present in all optical elements if they are inappropriately restrained in their holders. Larger optics (e.g. telescope windows) can exhibit inherent stress birefringence due to annealing and/or their own weight. Such optical elements can easily be visually inspected by means of crossed polarising sheet filters before and after the sample. Furthermore, non-parallel (converging or diverging) incident beams on optics with polarisation effects depending on the incidence angle will cause 
depolarisation. The manufacturer's specification of dedicated polarisation optics should be sufficient to determine the maximum allowable divergence of the incident beam, but, for example, the coatings of $90^{\circ}$ reflecting mirrors in Newtonian telescopes are usually not sufficiently specified to determine their polarisation effects. The depolarising effects of optics can additionally depend on the state of polarisation of the incident beam.

\section{Summary and conclusions}

The presented equations can be used to analyse the effects of polarising optics of a variety of lidar systems and to assess the accuracy and error of several calibration techniques. From the parameters of the optics, which are either given by the manufacturers or measured (some can be measured by means of procedures described in this work), we determine the general signal parameters $G_{S}$ and $H_{S}$ in Sect. 4 for the correction of the measured signals and the derived linear depolarisation ratio regarding polarisation effects of the lidar system. They are independent of the atmospheric depolarisation. Additionally we need calibration measurements for the unpolarised gain ratio of the cross to the parallel signal. They can depend on the atmospheric depolarisation and must also be corrected for polarisation effects of the lidar system as described in Sects. 5 to 10. We show how this dependence can be avoided or minimized and presented correction formulas for the gain ratio for all cases.

Major findings are that a cleaned analyser and no rotation of the receiving optics with respect to the laser polarisation avoid many error terms and allow the determination and correction of other misalignments and the optics diattenuation, and that the $\Delta 90$ calibration can decrease the error of a single $\pm 45^{\circ}$ calibration into insignificance.

We show that a linear polariser such as a calibrator should have a very good extinction ratio in order to avoid large calibration errors (Eq. 126). The advantage of a sheet polariser (and $\lambda / 4$ sheet filters) is its tenuity; therefore, it can be included in many existing lidar systems with minimal space requirement, for example with a sheet holder as shown in Fig. 5. Such a sheet holder guarantees an accurate $\Delta 90^{\circ}$ rotation of the sheet; therefore, the absolute accuracy of the $45^{\circ}$ orientation is not important. Together with an existing calibration technique or inserted at different positions, the filter holder can be used to determine the diattenuation of the optics between the two positions (see Eqs. 131 and 138-145). Furthermore, the determination of the calibration factor with an ideal linear polariser calibrator is always independent of changes of the input light and hence independent of the atmospheric depolarisation, in contrast to the other calibrators. Plastic sheet filters can easily be cut to be used in a rotation holder as in Fig. 6 so that the filter can be automatically rotated to $\Delta 90^{\circ}$ positions and out of the optical path for standard measurements. Large acceptance angles of linearly polarising sheet filters allows the mounting close to the telescope focus where we have some free space, and the filter diameter and mechanical mounting can be small due to the small beam diameter. However, it should be considered that the direction of the polarising structure of a sheet filter is not necessarily constant over the whole sheet, which is usually not specified by the manufacturers and should be inquired before the purchase.

$\lambda / 4$ plates and circular polariser made of sheet films have similar constraints. Furthermore, the $\Delta 90$ calibration does not work with a $\lambda / 4$ plate because the $\pm 45^{\circ}$ errors do not compensate (Eqs. 154 and 164), but in exchange we can determine with it the amount of circular polarisation (Eqs. 158 and 168). In contrast to that, the ideal circular polariser calibration 
does not depend on the rotation error $\varepsilon$ and the input light polarisation at all and does not need a $\Delta 90$ calibration, but inherent errors of a real circular polariser, which usually are not sufficiently specified by manufacturers, would be difficult to assess, and the resulting error equations are complex.

While all optical calibrators exhibit wavelength dependency and have the disadvantage of possible inhomogeneities over the surface and other optical errors such as inaccurate phase shift or crosstalk, which would add a system depolarisation, the only possible error source of the mechanical rotation calibrator (Sect. 7) is the accuracy of the rotation itself. Although more bulky, it is the most reliable calibrator if used with a cleaned analyser and accurate $\Delta 90^{\circ}$ rotation (Eq. 105). It is independent of wavelength, has no internal uncertainties, and is insensitive to temporal changes and degradation.
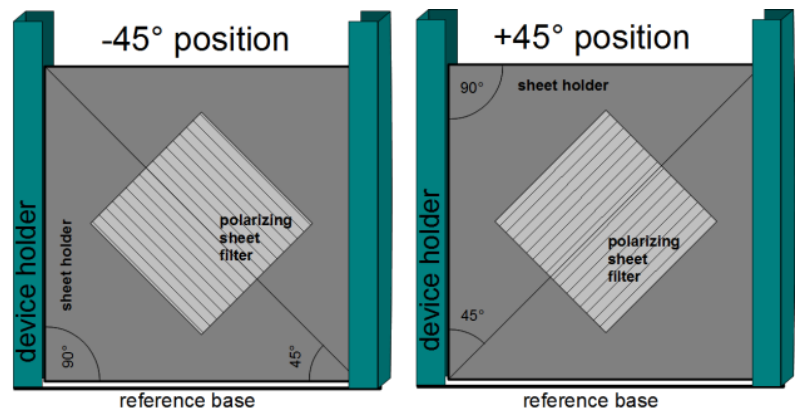

Figure 5. Simple holder for sheet filters (linear polariser or $\lambda / 4$ plate) with accurate positioning for the $\Delta 90$ calibration.
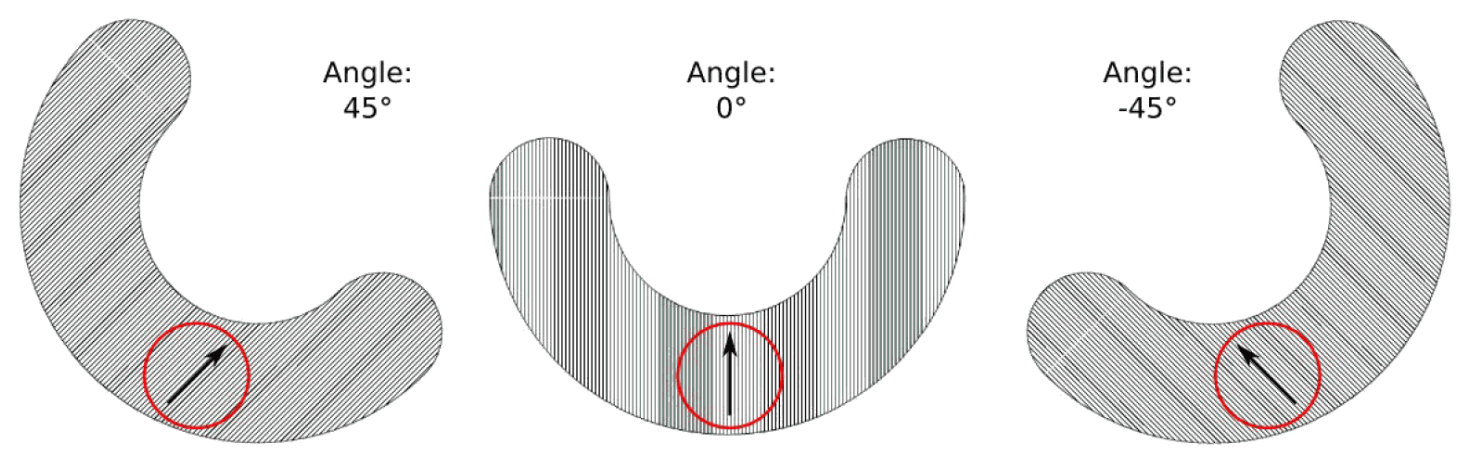

Figure 6. Linearly polarising sheet filter cut-out for use in a rotation mount. The optical axis of the filtered light beam is in the centre of the red circle. Reproduced with permission from Kölbl (2010). 


\section{Appendix A: Abbreviations and definitions}

$a$

$a_{L}$

$a^{\prime}$

$\alpha$

$\beta$

$\gamma$

$\mathrm{c}_{2 \varepsilon}$

$\delta$

$\delta *$

D

$\varepsilon$

$\eta_{T, R}$

$\eta$

$\eta *$

$\eta_{\Delta 90}^{*}$

I

I

LDR

F

$F_{i j}$

$G_{S}, H_{S}$

K

$\mathbf{M}_{S}, \mathbf{M}_{T, R}$

PBS

$T_{S}$

$T^{p}, T^{s}, R^{p}, R^{s}$

$Z_{O}$
Polarisation parameter of the atmospheric volume; see Eq. 9

Polarisation parameter of the light beam leaving the laser

$a^{\prime}=a a_{L}$, combined laser-atmosphere polarisation parameter

Rotation of the plane of horizontal linear polarisation of the laser around the $z$ axis (laser rotation)

Rotation of the emitter optics around the $z$ axis

Rotation of the receiver optics around the $z$ axis

$\cos (2 \varepsilon)$

Volume linear depolarisation ratio of the atmospheric scattering volume; see Eq. (12)

Calibrated signal ratio, but including crosstalk and alignment errors

Diattenuation parameter (see Sect. 2.2)

Error angle of the $\Delta 90$ calibration setup

Electronic amplification of individual transmitted/reflected channels

$\eta=\eta_{R} T_{R} / \eta_{T} T_{T}$ calibration factor including only the electronic amplification and the optical diattenuation of the polarising beam splitter

Gain ratio, i.e. the measured, apparent calibration factor $\eta^{*}$ of the polarisation channels, i.e. the calibration factor $\eta$ including the crosstalk from optics before the polarising beam splitter and from system alignment errors

$\Delta 90$ gain ratio $\eta_{\Delta 90}^{*} \equiv \sqrt{\eta^{*}\left(+45^{\circ}+\varepsilon\right) \eta^{*}\left(-45^{\circ}+\varepsilon\right)}$; measured, apparent calibration factor retrieved with the $\Delta 90$ calibration method

Power/flux of the light beam (watt/lumen) (colloquially: intensity)

Stokes vector of the light beam

Linear depolarisation ratio $=\delta$

Müller matrix of the atmospheric scattering volume in backscattering direction

Element $i j$ of $\mathbf{F}$

Crosstalk parameters (Sect. 4.1)

Correction of the measured gain ratio $\eta^{*}$ to get the calibration factor $\eta$ (Sect. 5)

Müller matrix of the polarising beam splitter $s$, e.g. a polarising beam splitter cube, in the transmission $_{T}$ and reflection ${ }_{R}$ path.

Polarising beam splitter

Transmission of matrix $\mathbf{M}_{S}$ for unpolarised light (alias average transmission)

Intensity transmission and reflection coefficients of the polarising beam splitter for parallel $p$ and perpendicular $s$ linearly polarised light with respect to the plane of incidence.

$Z_{O}=\sqrt{1-D_{o}^{2}}$ 


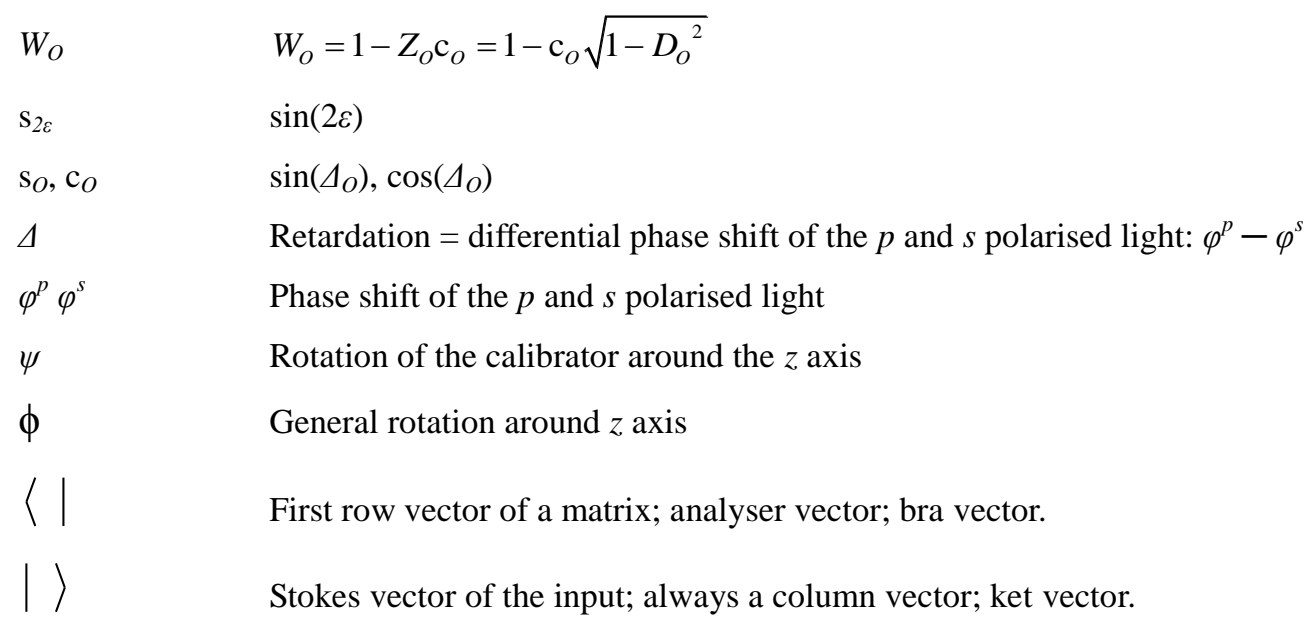

Setup parameters:

$\mathrm{h}$

$\mathrm{X}, \mathrm{z}$

$\mathrm{y}$
Binary operator to select either manual rotation $(h=+1)$ or rotation by means of a $\lambda / 2$ plate $(h=-1)$

Binary operators to select calibration angles of $+45^{\circ}(\mathrm{x}, \mathrm{z}=+1)$ or $-45^{\circ}(\mathrm{x}, \mathrm{z}=-1)$

Binary operator to select standard measurement setup angles of $+0^{\circ}(\mathrm{y}=+1)$ or $+90^{\circ}(\mathrm{y}=-1)$

\section{Appendix B: The <bra|ket $>$ notation}

Superscript ${ }^{\mathrm{T}}$ means the transposition of a row vector to a column vector and vice versa, while the $\mid$ ket $>$ and $<$ bra| vector symbols always stand for a column vector and row vector, respectively. That means

$$
\left(\begin{array}{l}
a \\
b \\
c \\
d
\end{array}\right)=\left(\begin{array}{llll}
a & b & c & d
\end{array}\right)^{T}=\left|\begin{array}{llll}
a & b & c & d
\end{array}\right\rangle=\left|\begin{array}{l}
a \\
b \\
c \\
d
\end{array}\right|
$$

are forms of column vectors, and

$$
\left(\begin{array}{llll}
a & b & c & d
\end{array}\right)=\left(\begin{array}{l}
a \\
b \\
c \\
d
\end{array}\right)^{T}=\left\langle\begin{array}{llll}
a & b & c & d
\end{array}\right|=\left(\begin{array}{l}
a \\
b \\
c \\
d
\end{array} \mid\right.
$$

are forms of row vectors. 


\section{Appendix C: The calibration equation}

The general equation for the calibration signals in Eq. (81) can be written similarly to that in Kaul et al. (2004) using general expressions for the analyser row vector $\left\langle\mathbf{A}_{S}\right|$ (see Appendix D) and for the input Stokes vector $\left|\boldsymbol{I}_{\text {in }}\right\rangle$ (see Appendix E) as in Eq. (C1), irrespective of the actual position of the calibrator.

$\frac{I_{S}}{\eta_{S}}=\left\langle\mathbf{A}_{S}\left|\mathbf{C}\left(\mathrm{x} 45^{\circ}+\varepsilon\right)\right| \boldsymbol{I}_{\text {in }}\right\rangle=I_{i n}\left\langle\begin{array}{c}A_{S}^{1} \\ A_{S}^{2} \\ A_{S}^{3} \\ A_{S}^{4}\end{array}\left|\mathbf{C}\left(\mathrm{x} 45^{\circ}+\varepsilon\right)\right| \begin{array}{c}i_{\text {in }} \\ q_{\text {in }} \\ u_{\text {in }} \\ v_{\text {in }}\end{array}\right\rangle$

For certain setups the fully expanded equations are very complex. But sometimes slightly expanded versions are sufficient to achieve significant insights. Demerging the $\left( \pm 45^{\circ}+\varepsilon\right)$ rotation from the calibrator, as in Eq. (C2), or just the $\varepsilon$ - rotations, as in Eq. (C3), and applying the appropriate parts to the analyser and to the input Stokes vector can help to show general relations. For this purpose we define the rotated analyser vector $\left\langle\mathbf{A}_{S, \varepsilon}\right|$ and the rotated input Stokes vector $\mid \boldsymbol{I}_{i, \varepsilon}>$ as shown in Eq. (C3).

$$
\begin{aligned}
& I_{S}=\eta_{S}\left\langle\mathbf{A}_{S}\left|\mathbf{C}\left(\mathrm{x} 45^{\circ}+\varepsilon\right)\right| \boldsymbol{I}_{\text {in }}\right\rangle= \\
& =\eta_{S}\left\langle\mathbf{A}_{S}\left|\mathbf{R}\left(\mathrm{x} 45^{\circ}+\varepsilon\right) \mathbf{C}(0) \mathbf{R}\left(-\mathrm{x} 45^{\circ}-\varepsilon\right)\right| \boldsymbol{I}_{\text {in }}\right\rangle \Rightarrow \\
& \frac{I_{S}}{\eta_{S} I_{i n}}=\left(\begin{array}{c}
A_{S}^{1} \\
A_{S}^{2} \\
A_{S}^{3} \\
A_{S}^{4}
\end{array}\left(\begin{array}{cccc}
1 & 0 & 0 & 0 \\
0 & -\mathrm{xs}_{2 \varepsilon} & -\mathrm{xc}_{2 \varepsilon} & 0 \\
0 & \mathrm{xc}_{2 \varepsilon} & -\mathrm{xs}_{2 \varepsilon} & 0 \\
0 & 0 & 0 & 1
\end{array}\right) \mathbf{C}(0)\left(\begin{array}{cccc}
1 & 0 & 0 & 0 \\
0 & -\mathrm{xs}_{2 \varepsilon} & \mathrm{xc}_{2 \varepsilon} & 0 \\
0 & -\mathrm{xc}_{2 \varepsilon} & -\mathrm{xs}_{2 \varepsilon} & 0 \\
0 & 0 & 0 & 1
\end{array}\right) \mid \begin{array}{l}
i_{i n} \\
q_{i n} \\
u_{i n} \\
v_{\text {in }}
\end{array}\right)= \\
& =\left\langle\begin{array}{c}
A_{S}^{1} \\
\mathrm{x}\left(\mathrm{c}_{2 \varepsilon} A_{S}^{3}-\mathrm{s}_{2 \varepsilon} A_{S}^{2}\right) \\
-\mathrm{x}\left(\mathrm{c}_{2 \varepsilon} A_{S}^{2}+\mathrm{s}_{2 \varepsilon} A_{S}^{3}\right) \\
A_{S}^{4}
\end{array}|\mathbf{C}(0)| \begin{array}{c}
i_{i n} \\
\mathrm{x}\left(\mathrm{c}_{2 \varepsilon} u_{i n}-\mathrm{s}_{2 \varepsilon} q_{i n}\right) \\
-\mathrm{x}\left(\mathrm{c}_{2 \varepsilon} q_{i n}+\mathrm{s}_{2 \varepsilon} u_{i n}\right) \\
v_{i n}
\end{array}\right)=\left\langle\begin{array}{c}
A_{S}^{1} \\
\mathrm{x} A_{S, \varepsilon}^{3} \\
-\mathrm{x} A_{S, \varepsilon}^{2} \\
A_{S}^{4}
\end{array}|\mathbf{C}(0)| \begin{array}{c}
i_{i n} \\
\mathrm{x} u_{i n, \varepsilon} \\
-\mathrm{x} q_{i n, \varepsilon} \\
v_{i n}
\end{array}\right\rangle
\end{aligned}
$$




$$
\begin{aligned}
& I_{S}=\eta_{S}\left\langle\mathbf{A}_{S}\left|\mathbf{C}\left(\mathrm{x} 45^{\circ}+\varepsilon\right)\right| \boldsymbol{I}_{\text {in }}\right\rangle= \\
& =\eta_{S}\left\langle\mathbf{A}_{S}\left|\mathbf{R}(+\varepsilon) \mathbf{C}\left(\mathrm{x} 45^{\circ}\right) \mathbf{R}(-\varepsilon)\right| \boldsymbol{I}_{i n}\right\rangle=\eta_{S}\left\langle\mathbf{A}_{S, \varepsilon}\left|\mathbf{C}\left(\mathrm{x} 45^{\circ}\right)\right| \boldsymbol{I}_{i n, \varepsilon}\right\rangle \Rightarrow \\
& \frac{I_{S}}{\eta_{S} I_{\text {in }}}=\left(\begin{array}{c}
A_{S}^{1} \\
A_{S}^{2} \\
A_{S}^{3} \\
A_{S}^{4}
\end{array}\left(\begin{array}{cccc}
1 & 0 & 0 & 0 \\
0 & \mathrm{c}_{2 \varepsilon} & -\mathrm{s}_{2 \varepsilon} & 0 \\
0 & \mathrm{~s}_{2 \varepsilon} & \mathrm{c}_{2 \varepsilon} & 0 \\
0 & 0 & 0 & 1
\end{array}\right) \mathbf{C}\left(\times 45^{\circ}\right)\left(\begin{array}{cccc}
1 & 0 & 0 & 0 \\
0 & \mathrm{c}_{2 \varepsilon} & \mathrm{s}_{2 \varepsilon} & 0 \\
0 & -\mathrm{s}_{2 \varepsilon} & \mathrm{c}_{2 \varepsilon} & 0 \\
0 & 0 & 0 & 1
\end{array}\right) \mid \begin{array}{l}
i_{i n} \\
q_{i n} \\
u_{i n} \\
v_{\text {in }}
\end{array}\right)= \\
& =\left\langle\begin{array}{c}
A_{S}^{1} \\
\mathrm{c}_{2 \varepsilon} A_{S}^{2}+\mathrm{s}_{2 \varepsilon} A_{S}^{3} \\
\mathrm{c}_{2 \varepsilon} A_{S}^{3}-\mathrm{s}_{2 \varepsilon} A_{S}^{2} \\
A_{S}^{4}
\end{array}\left|\mathbf{C}\left(\mathrm{x} 45^{\circ}\right)\right| \begin{array}{c}
i_{i n} \\
\mathrm{c}_{2 \varepsilon} q_{i n}+\mathrm{s}_{2 \varepsilon} u_{i n} \\
\mathrm{c}_{2 \varepsilon} u_{i n}-\mathrm{s}_{2 \varepsilon} q_{i n} \\
v_{i n}
\end{array}\right| \equiv\left\langle\begin{array}{c}
A_{S}^{1} \\
A_{S, \varepsilon}^{2} \\
A_{S, \varepsilon}^{3} \\
A_{S}^{4}
\end{array}\left|\mathbf{C}\left(\mathrm{x} 45^{\circ}\right)\right| \begin{array}{c}
i_{i n} \\
q_{i n, \varepsilon} \\
u_{i n, \varepsilon} \\
v_{i n}
\end{array}\right\rangle
\end{aligned}
$$

Note the exchange of places of $A_{S, \varepsilon}^{2}$ and $A_{S, \varepsilon}^{3}$ and of $q_{i n, \varepsilon}$ and $u_{i n, \varepsilon}$ between Eqs. (C2) and (C3).

\section{C1 Calibration with a rotator}

From Eqs. (C1), (C3), and (S.10.15.2) we get the general calibration signals in Eq. (C4) with analyser vectors $<\mathbf{A} \mid$ from Appendix D and input Stokes vectors $\mid \boldsymbol{I}_{i n}>$ from Appendix E.

$$
\begin{aligned}
& \frac{I_{S}}{\eta_{S} I_{\text {in }}}=\frac{\left\langle\mathbf{A}_{S}\left|\mathbf{M}_{\text {rot }}\left(\mathrm{x} 45^{\circ}+\varepsilon, \mathrm{h}\right)\right| \boldsymbol{I}_{\text {in }}\right\rangle}{I_{\text {in }}}= \\
& =\left\langle\begin{array}{c}
A_{S}^{1} \\
A_{S}^{2} \\
A_{S}^{3} \\
A_{S}^{4}
\end{array}\left|\left(\begin{array}{cccc}
1 & 0 & 0 & 0 \\
0 & -\mathrm{xs}_{2 \varepsilon} & -\mathrm{xhc}_{2 \varepsilon} & 0 \\
0 & \mathrm{xc}_{2 \varepsilon} & -\mathrm{xhs}_{2 \varepsilon} & 0 \\
0 & 0 & 0 & \mathrm{~h}
\end{array}\right)\right| \begin{array}{l}
i_{i n} \\
q_{i n} \\
u_{i n} \\
v_{i n}
\end{array}\right\rangle= \\
& =A_{S}^{1} i_{i n}+A_{S}^{4} \mathrm{~h} v_{i n}-\mathrm{x}\left[\left(\mathrm{s}_{2 \varepsilon} A_{S}^{2}-\mathrm{c}_{2 \varepsilon} A_{S}^{3}\right) q_{i n}+\left(\mathrm{c}_{2 \varepsilon} A_{S}^{2}+\mathrm{s}_{2 \varepsilon} A_{S}^{3}\right) \mathrm{h} u_{i n}\right]= \\
& =A_{S}^{1} i_{i n}+A_{S}^{4} \mathrm{~h} v_{i n}+\mathrm{x}\left[\begin{array}{llll}
A_{S, \varepsilon}^{3} q_{i n} & - & A_{S, \varepsilon}^{2} \mathrm{~h} u_{\text {in }}
\end{array}\right]
\end{aligned}
$$

\section{C2 Calibration with a linear polariser}

From Eqs. (C3) and (S.10.7.1) we get the general calibration signals in Eq. (C5) with analyser vectors <A| from Appendix D and input Stokes $\left|\boldsymbol{I}_{\text {in }}\right\rangle$ vectors from Appendix E. With an ideal linear polariser Eq. (C5) reduces to Eq. (C6). 


$$
\begin{aligned}
& I_{S}=\eta_{S}\left\langle\mathbf{A}_{S}\left|\mathbf{M}_{P}\left(\mathrm{x} 45^{\circ}+\varepsilon\right)\right| \boldsymbol{I}_{i n}\right\rangle=\eta_{S}\left\langle\mathbf{A}_{S}\left|\mathbf{R}(+\varepsilon) \mathbf{M}_{P}\left(\mathrm{x} 45^{\circ}\right) \mathbf{R}(-\varepsilon)\right| \boldsymbol{I}_{i n}\right\rangle \Rightarrow \\
& \left.\left.\frac{I_{S}}{\eta_{S} T_{P} I_{i n}}=\left\langle\begin{array}{c}
A_{S}^{1} \\
A_{S, \varepsilon}^{2} \\
A_{S, \varepsilon}^{3} \\
A_{S}^{4}
\end{array}\right| \begin{array}{cccc}
1 & 0 & \mathrm{x} D_{P} & 0 \\
0 & Z_{P} \mathrm{c}_{P} & 0 & -\mathrm{x}_{P} \mathrm{~s}_{P} \\
\mathrm{x} D_{P} & 0 & 1 & 0 \\
0 & \mathrm{x} Z_{P} \mathrm{~s}_{P} & 0 & Z_{P} \mathrm{c}_{P}
\end{array}\right) \mid \begin{array}{c}
i_{i n} \\
q_{i n, \varepsilon} \\
u_{i n, \varepsilon} \\
v_{i n}
\end{array}\right)= \\
& =\left\{\begin{array}{l}
A_{S}^{1} i_{i n}+A_{S, \varepsilon}^{3} u_{i n, \varepsilon}+Z_{P} \mathrm{c}_{P}\left(A_{S, \varepsilon}^{2} q_{i n, \varepsilon}+A_{S}^{4} v_{i n}\right)+ \\
+\mathrm{x}\left[D_{P}\left(A_{S}^{1} u_{i n, \varepsilon}+A_{S, \varepsilon}^{3} i_{i n}\right)-Z_{P} \mathrm{~s}_{P}\left(A_{S, \varepsilon}^{2} v_{i n}-A_{S}^{4} q_{i n, \varepsilon}\right)\right]
\end{array}\right\} \\
& D_{P}=1, Z_{P}=0 \Rightarrow \\
& \frac{I_{S}}{\eta_{S} T_{P} I_{i n}}=A_{S}^{1} i_{i n}+A_{S, \varepsilon}^{3} u_{i n, \varepsilon}+\mathrm{x}\left(A_{S}^{1} u_{i n, \varepsilon}+A_{S, \varepsilon}^{3} i_{i n}\right)=\left(A_{S}^{1}+\mathrm{x} A_{S, \varepsilon}^{3}\right)\left(i_{i n}+\mathrm{x} u_{i n, \varepsilon}\right)
\end{aligned}
$$

\section{C3 Calibration with a $\lambda / 4$ plate (QWP)}

From Eqs. (C2) and (S.10.11.1) for the $\lambda / 4$ plate with retardation error $\omega$ as in Eq. (C7) we get the general calibration signals in Eq. (C8) with analyser vectors $<\mathbf{A} \mid$ from Appendix D and input Stokes vectors $\mid \boldsymbol{I}_{\text {in }}>$ from Appendix E.

$$
\Delta_{Q W}=90^{\circ}+\omega \Rightarrow \mathrm{c}_{Q W}=-\mathrm{s}_{\omega}, \mathrm{s}_{Q W}=\mathrm{c}_{\omega}
$$

$\frac{I_{S}}{\eta_{S} T_{Q W} I_{\text {in }}}=\frac{\left\langle\mathbf{A}_{S}\left|\mathbf{M}_{Q W}\left(\mathrm{x} 45^{\circ}+\varepsilon, \omega\right)\right| \boldsymbol{I}_{\text {in }}\right\rangle}{T_{Q W} I_{\text {in }}}=$

$=\frac{\left\langle\mathbf{A}_{S}\left|\mathbf{R}\left(\mathrm{x} 45^{\circ}+\varepsilon\right) \mathbf{M}_{Q W}(0, \omega) \mathbf{R}\left(-\mathrm{x} 45^{\circ}-\varepsilon\right)\right| \boldsymbol{I}_{\text {in }}\right\rangle}{T_{Q W} I_{\text {in }}}=$

$=\left\langle\begin{array}{c}A_{S}^{1} \\ \mathrm{x} A_{S, \varepsilon}^{3} \\ -\mathrm{x} A_{S, \varepsilon}^{2} \\ A_{S}^{4}\end{array}\left|\left(\begin{array}{cccc}1 & 0 & 0 & 0 \\ 0 & 1 & 0 & 0 \\ 0 & 0 & -\mathrm{s}_{\omega} & \mathrm{c}_{\omega} \\ 0 & 0 & \mathrm{c}_{\omega} & -\mathrm{s}_{\omega}\end{array}\right)\right| \begin{array}{c}i_{i n} \\ \mathrm{x} u_{i n, \varepsilon} \\ -\mathrm{x} q_{i n, \varepsilon} \\ v_{i n}\end{array}\right\rangle=$

$=A_{S}^{1} i_{i n}+A_{S, \varepsilon}^{3} u_{i n, \varepsilon}-\mathrm{s}_{\omega}\left(A_{S, \varepsilon}^{2} q_{i n, \varepsilon}+A_{S}^{4} v_{i n}\right)-\mathrm{xc}_{\omega}\left(A_{S}^{4} q_{i n, \varepsilon}+A_{S, \varepsilon}^{2} v_{i n}\right)$

$\omega=0 \Rightarrow$

$\frac{I_{S}}{\eta_{S} T_{Q W} I_{i n}}=\frac{\left\langle\mathbf{A}_{S}\left|\mathbf{M}_{Q W}\left(\mathrm{x} 45^{\circ}+\varepsilon, 0\right)\right| \boldsymbol{I}_{i n}\right\rangle}{T_{Q W} I_{i n}}=A_{S}^{1} i_{i n}+A_{S, \varepsilon}^{3} u_{i n, \varepsilon}-\mathrm{x}\left(A_{S}^{4} q_{i n, \varepsilon}+A_{S, \varepsilon}^{2} v_{i n}\right)=$

$=A_{S}^{1} i_{i n}-\left(\mathrm{s}_{2 \varepsilon} A_{S}^{2}-\mathrm{c}_{2 \varepsilon} A_{S}^{3}\right)\left(\mathrm{s}_{2 \varepsilon} q_{i n}-\mathrm{c}_{2 \varepsilon} u_{i n}\right)-\mathrm{x}\left[A_{S}^{4}\left(\mathrm{c}_{2 \varepsilon} q_{i n}+\mathrm{s}_{2 \varepsilon} u_{i n}\right)+\left(\mathrm{c}_{2 \varepsilon} A_{S}^{2}+\mathrm{s}_{2 \varepsilon} A_{S}^{3}\right) v_{i n}\right]$ 


\section{C4 Calibration with a circular polariser (CP)}

From Eq. (C2) for a circular polariser composed of a linear polariser and a $\lambda / 4$ plate with retardation error $\omega$ as in Eq. (C7) we get the general calibration signals in Eq. (C10) with analyser vectors $<\mathbf{A} \mid$ from Appendix D and input Stokes vectors $\mid \boldsymbol{I}_{\text {in }}>$ from Appendix E. Note that $\mathrm{z}= \pm 1$ discerns between a right and left circular polariser, and $\mathrm{x}= \pm 1$ between the $\pm 45^{\circ}$ orientations of the whole circular polariser. With an ideal linear polariser this quite complex equation reduces to Eq. (C11), with an ideal QWP without retardation error to Eq. (C12), and to Eq. (C13) with both constraints, i.e. for an ideal circular polariser. Since only the terms with an $\mathrm{x}$ in Eqs. (C11) to (C13) are compensated by means of the $\Delta 90$ calibration, neither of the two constraints alone is sufficient to reduce the uncertainty.

$$
\begin{aligned}
& \frac{I_{S}}{\eta_{S} T_{C P} I_{\text {in }}}=\frac{\left\langle\mathbf{A}_{S}\left|\mathbf{M}_{C P}\left(\mathrm{z}, \mathrm{X} 45^{\circ}+\varepsilon\right)\right| \boldsymbol{I}_{\text {in }}\right\rangle}{T_{C P} I_{\text {in }}}= \\
& =\frac{\left\langle\mathbf{A}_{S} \mathbf{R}\left(\mathrm{x} 45^{\circ}+\varepsilon\right)\left|\mathbf{M}_{Q W}\left(\mathrm{z} 45^{\circ}, \omega\right) \mathbf{M}_{P}\right| \mathbf{R}\left(-\mathrm{x} 45^{\circ}-\varepsilon\right) \boldsymbol{I}_{\text {in }}\right\rangle}{T_{Q W} T_{P} I_{\text {in }}}= \\
& =\left(\begin{array}{c}
A_{S}^{1} \\
\mathrm{x} A_{S, \varepsilon}^{3} \\
-\mathrm{x} A_{S, \varepsilon}^{2} \\
A_{S}^{4}
\end{array}\left|\left(\begin{array}{cccc}
1 & 0 & 0 & 0 \\
0 & -\mathrm{s}_{\omega} & 0 & -\mathrm{zc}_{\omega} \\
0 & 0 & 1 & 0 \\
0 & \mathrm{zc}_{\omega} & 0 & -\mathrm{s}_{\omega}
\end{array}\right)\left(\begin{array}{cccc}
1 & D_{P} & 0 & 0 \\
D_{P} & 1 & 0 & 0 \\
0 & 0 & Z_{P} \mathrm{c}_{P} & Z_{P} \mathrm{~s}_{P} \\
0 & 0 & -Z_{P} \mathrm{~s}_{P} & Z_{P} \mathrm{c}_{P}
\end{array}\right)\right| \begin{array}{c}
i_{i n} \\
\mathrm{x} u_{i n, \varepsilon} \\
-\mathrm{x} q_{i n, \varepsilon} \\
v_{i n}
\end{array}\right)= \\
& =\left\{\begin{array}{l}
\left(A_{S}^{1}+A_{S}^{4} \mathrm{zc}_{\omega} D_{P}\right) i_{i n}+\left(A_{S, \varepsilon}^{2} Z_{P} \mathrm{c}_{P}-\mathrm{z} A_{S, \varepsilon}^{3} \mathrm{c}_{\omega} Z_{P} \mathrm{~s}_{P}\right) q_{i n, \varepsilon}-A_{S, \varepsilon}^{3} \mathrm{~s}_{\omega} u_{i n, \varepsilon}-A_{S}^{4} \mathrm{~s}_{\omega} Z_{P} \mathrm{c}_{P} v_{i n}+ \\
+\mathrm{x}\left[\begin{array}{l}
-A_{S, \varepsilon}^{3} \mathrm{~s}_{\omega} D_{P} i_{i n}-A_{S}^{4} \mathrm{~s}_{\omega} Z_{P} \mathrm{~s}_{P} q_{i n, \varepsilon}+ \\
+\left(A_{S}^{1} D_{P}+A_{S}^{4} \mathrm{zc}_{\omega}\right) u_{i n, \varepsilon}-\left(A_{S, \varepsilon}^{2} \mathrm{~s}_{P}+A_{S, \varepsilon}^{3} \mathrm{zc}_{\omega} \mathrm{c}_{P}\right) Z_{P} v_{i n}
\end{array}\right]
\end{array}\right\}
\end{aligned}
$$

From Eq. (C10) we get under different conditions Eqs. (C11) to (C13).

$$
\begin{aligned}
& D_{P}=1, Z_{P}=0 \Rightarrow \\
& \frac{I_{S}}{\eta_{S} T_{C P} I_{i n}}=\left(A_{S}^{1}+\mathrm{zc}_{\omega} A_{S}^{4}-\mathrm{s}_{\omega} \mathrm{x} A_{S, \varepsilon}^{3}\right)\left(i_{i n}+\mathrm{x} u_{i n, \varepsilon}\right) \\
& \omega=0 \Rightarrow \\
& \frac{I_{S}}{\eta_{S} T_{C P} I_{i n}}=\left\{\begin{array}{l}
\left(A_{S}^{1}+A_{S}^{4} \mathrm{z} D_{P}\right) i_{i n}+\left(A_{S, \varepsilon}^{2} Z_{P} \mathrm{c}_{P}-\mathrm{z} A_{S, \varepsilon}^{3} Z_{P} \mathrm{~s}_{P}\right) q_{i n, \varepsilon} \\
+\mathrm{x}\left[\left(A_{S}^{1} D_{P}+\mathrm{z} A_{S}^{4}\right) u_{i n, \varepsilon}-\left(A_{S, \varepsilon}^{2} \mathrm{~s}_{P}+\mathrm{z} A_{S, \varepsilon}^{3} \mathrm{c}_{P}\right) Z_{P} v_{i n}\right]
\end{array}\right\} \\
& \omega=0, D_{P}=1, Z_{P}=0 \Rightarrow \\
& \frac{I_{S}}{\eta_{S} T_{C P} I_{i n}}=\left(A_{S}^{1}+\mathrm{z} A_{S}^{4}\right)\left(i_{i n}-\mathrm{x} u_{i n, \varepsilon}\right)
\end{aligned}
$$


Appendix D: The analyser row vector $<A_{S}$

The general formulation for the Stokes vector of a standard lidar signal $\boldsymbol{I}_{S}$ at the detector in the reflected channel, $\boldsymbol{I}_{R}$, and transmitted channel, $\boldsymbol{I}_{T}$, is

$$
\boldsymbol{I}_{S}=\eta_{S} \mathbf{M}_{S} \mathbf{R}_{\mathrm{y}} \mathbf{M}_{O}(\gamma) \mathbf{F}(a) \mathbf{M}_{E}(\beta) \boldsymbol{I}_{L}
$$

Only the first Stokes parameter is directly measured, and therefore we can reduce the complexity of the full matrix equations to an inner product between the analyser row vector $\left\langle\mathbf{A}_{S}\right|$ and the input Stokes column vector $\boldsymbol{I}_{\text {in }}$ similar to Kaul et al. (1992) and Volkov et al. (2015) as in Eq. (D2):

$$
I_{S}=\left\langle\mathbf{A}_{S} \mid \boldsymbol{I}_{\text {in }}\right\rangle
$$

In the case of a calibration measurement, we place a calibrator with matrix $\mathbf{C}$ between the input Stokes vector and the analyser vector:

$$
I_{S}=\left\langle\mathbf{A}_{S}|\mathbf{C}| \boldsymbol{I}_{\text {in }}\right\rangle
$$

As calibrators we use a mechanical rotator, a rotation of the plane of polarisation by means of a $\lambda / 2$ plate (HWP), a linear polariser, a $\lambda / 4$ plate (QWP), and a circular polariser. We can place the calibrator anywhere in the optical setup, with different results. In the following we develop the general expressions of the analyser vector in Appendix D and of the input Stokes vector in Appendix E for the different setups.

\section{D1 $\angle A_{S} \mid$ with $\mathrm{C}$ before the polarising beam splitter}

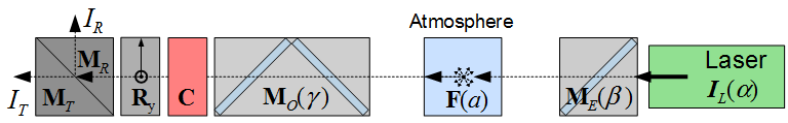

$$
\boldsymbol{I}_{S}=\eta_{S} \mathbf{M}_{S} \mathbf{R}_{\mathrm{y}} \mathbf{C} \mathbf{M}_{O} \mathbf{F} \boldsymbol{I}_{E} \Rightarrow \mathbf{A}_{S}=\mathbf{M}_{S} \mathbf{R}_{\mathrm{y}}
$$

The analyser part consists of a polarising beam splitter $\mathbf{M}_{S}$ and an optional $90^{\circ}$ rotation of the detector setup $\mathbf{R}_{\mathrm{y}}$ (see Eq. 47)

$$
\begin{aligned}
& \frac{\left\langle\mathbf{A}_{S}\right|}{T_{S}}=\frac{\left\langle\mathbf{M}_{S} \mathbf{R}_{\mathrm{y}}\right|}{T_{S}}= \\
& =\left\langle\left(\begin{array}{cccc}
1 & D_{S} & 0 & 0 \\
D_{S} & 1 & 0 & 0 \\
0 & 0 & Z_{S} \mathrm{c}_{S} & Z_{S} \mathrm{~s}_{S} \\
0 & 0 & -Z_{S} \mathrm{~s}_{S} & Z_{S} \mathrm{c}_{S}
\end{array}\right)\left(\begin{array}{cccc}
1 & 0 & 0 & 0 \\
0 & \mathrm{y} & 0 & 0 \\
0 & 0 & \mathrm{y} & 0 \\
0 & 0 & 0 & 1
\end{array}\right)\right|=\left\langle\left(\begin{array}{cccc}
1 & \mathrm{y} D_{S} & 0 & 0 \\
D_{S} & \mathrm{y} & 0 & 0 \\
0 & 0 & \mathrm{y} Z_{S} \mathrm{c}_{S} & Z_{S} \mathrm{~s}_{S} \\
0 & 0 & -\mathrm{y} Z_{S} \mathrm{~s}_{S} & Z_{S} \mathrm{c}_{S}
\end{array}\right)\right|=\left(\begin{array}{c}
1 \\
\mathrm{y} D_{S} \\
0 \\
0
\end{array} \mid\right.
\end{aligned}
$$


D2 $<A_{S} \mid$ with $\mathrm{C}$ before the receiving optics

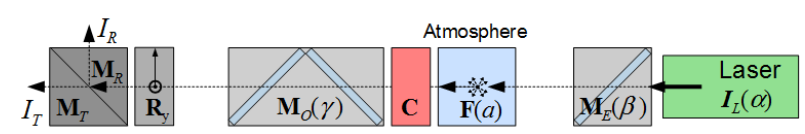

$\boldsymbol{I}_{S}=\eta_{S} \mathbf{M}_{S} \mathbf{R}_{\mathrm{y}} \mathbf{M}_{O} \mathbf{C F I} \boldsymbol{I}_{E} \Rightarrow \mathbf{A}_{S}=\mathbf{M}_{S} \mathbf{R}_{\mathrm{y}} \mathbf{M}_{O}$

Using Eq. (D5) we get

$$
\begin{aligned}
& \frac{\left\langle\mathbf{A}_{S}(\mathrm{y}, \gamma)\right|}{T_{O} T_{S}}=\frac{\left\langle\mathbf{M}_{S} \mathbf{R}_{\mathrm{y}}\right| \mathbf{M}_{O}(\gamma)}{T_{O} T_{S}}= \\
& =\left(\begin{array}{c}
1 \\
\mathrm{y} D_{S} \\
0 \\
0
\end{array} \mid\left(\begin{array}{cccc}
1 & \mathrm{c}_{2 \gamma} D_{O} & \mathrm{~s}_{2 \gamma} D_{O} & 0 \\
\mathrm{c}_{2 \gamma} D_{O} & 1-\mathrm{s}_{2 \gamma}^{2} W_{O} & \mathrm{~s}_{2 \gamma} \mathrm{c}_{2 \gamma} W_{O} & -\mathrm{s}_{2 \gamma} Z_{O} \mathrm{~s}_{O} \\
\mathrm{~s}_{2 \gamma} D_{O} & \mathrm{~s}_{2 \gamma} \mathrm{c}_{2 \gamma} W_{O} & 1-\mathrm{c}_{2 \gamma}^{2} W_{O} & \mathrm{c}_{2 \gamma} Z_{O} \mathrm{~s}_{O} \\
0 & \mathrm{~s}_{2 \gamma} Z_{O} \mathrm{~s}_{O} & -\mathrm{c}_{2 \gamma} Z_{O} \mathrm{~s}_{O} & Z_{O} \mathrm{c}_{O}
\end{array}\right)=\left(\begin{array}{c}
1+\mathrm{yc}_{2 \gamma} D_{O} D_{S} \\
\mathrm{c}_{2 \gamma} D_{O}+\mathrm{y} D_{S}\left(1-\mathrm{s}_{2 \gamma}^{2} W_{O}\right) \\
\mathrm{s}_{2 \gamma}\left(D_{O}+\mathrm{yc}_{2 \gamma} D_{S} W_{O}\right) \\
-\mathrm{ys}_{2 \gamma} D_{S} Z_{O} \mathrm{~s}_{O}
\end{array} \mid\right.\right.
\end{aligned}
$$

Simplifications: A rotation $\gamma$ of a retarding diattenuator $\mathbf{M}_{O}$ between the calibrator and the polarising beam splitter $\mathbf{M}_{S}$ complicates the equations considerably. In the case that $\mathbf{M}_{O}$ is not rotated $(\gamma=0)$, the matrices $\mathbf{M}_{S}$, the optional $90^{\circ}$ rotation $\mathbf{R}_{\mathrm{y}}$, and $\mathbf{M}_{O}$ can be combined to a new polarising beam splitter module $\mathbf{M}_{S \mathrm{y} O}$ according to Supplement Sect. S10.10, and all equations developed for the Sect. 7.1 case can be applied in Sect. 7.2. For $\gamma=0^{\circ}$ Eq. (D7) becomes $\gamma=0^{\circ} \Rightarrow$

$$
\begin{aligned}
& \left\langle\mathbf{A}_{S}\right|\left(\mathrm{y}, 0^{\circ}\right)=\left\langle\mathbf{M}_{S} \mathbf{R}_{\mathrm{y}}\right| \mathbf{M}_{O}\left(0^{\circ}\right)=T_{O} T_{S}\left\langle 1+\mathrm{y} D_{S} D_{O} \quad D_{O}+\mathrm{y} D_{S} \quad 0 \quad 0\right|= \\
& =\left\langle\mathbf{M}_{S \mathrm{y} O}\left(0^{\circ}\right)\right|=T_{S \mathrm{y} O}\left\langle\begin{array}{llll}
1 & D_{S \mathrm{y} O} & 0 & 0
\end{array}\right|
\end{aligned}
$$

with $T_{\text {SyO }}=T_{O} T_{S}\left(1+\mathrm{y} D_{S} D_{O}\right)$ and $D_{S y O}=\frac{D_{O}+\mathrm{y} D_{S}}{1+\mathrm{y} D_{S} D_{O}}$

With a cleaned analyser we get from Eq. (D9)

$$
\begin{aligned}
& D_{R}=-1, D_{T}=+1 \Rightarrow \\
& D_{S \mathrm{y} O}=y D_{S}, \quad D_{R \mathrm{y} O}=-y, \quad D_{T \mathrm{y} O}=+y \\
& T_{R \mathrm{y} O}=T_{O} T_{R}\left(1-\mathrm{y} D_{O}\right), \quad T_{T \mathrm{y} O}=T_{O} T_{T}\left(1+\mathrm{y} D_{O}\right)
\end{aligned}
$$

and explicitly with Eqs. (S.10.10.11) and (S.10.10.14) 


$$
\begin{array}{ll}
D_{R}=-1, D_{T}=+1, y=+1 \Rightarrow \\
T_{R+O}=T_{O} T_{R}\left(1-D_{O}\right)=0.5 T_{R}^{s} k_{1} T_{O}^{s}, & D_{R+O}=-1 \\
T_{T+O}=T_{O} T_{T}\left(1+D_{O}\right)=0.5 T_{T}^{p} k_{1} T_{O}^{p}, & D_{T+O}=+1 \\
D_{R}=-1, D_{T}=+1, y=-1 \Rightarrow & \\
T_{R-O}=T_{O} T_{R}\left(1+D_{O}\right)=0.5 T_{R}^{s} k_{1} T_{O}^{p}, & D_{R-O}=+1 \\
T_{T-O}=T_{O} T_{T}\left(1-D_{O}\right)=0.5 T_{T}^{p} k_{1} T_{O}^{s}, & D_{T-O}=-1
\end{array}
$$

See also Supplement Sects. S10.10 and S6.

Only few special cases with rotated $\mathbf{M}_{O}(\gamma \neq 0)$ (see Eq. S.5.1.4) are discussed additionally.

\section{D3 $<A_{S} \mid$ with $\mathrm{C}$ behind the emitter optics}

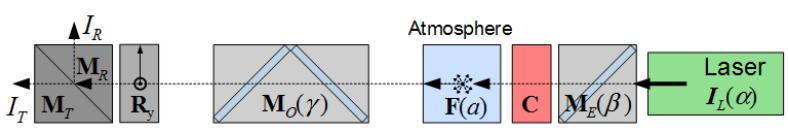

$$
\boldsymbol{I}_{S}=\eta_{S} \mathbf{M}_{S} \mathbf{R}_{\mathrm{y}} \mathbf{M}_{O} \mathbf{F C I _ { E }} \Rightarrow \mathbf{A}_{S}=\mathbf{M}_{S} \mathbf{R}_{\mathrm{y}} \mathbf{M}_{O} \mathbf{F C} \text { and } \boldsymbol{I}_{\text {in }}=\boldsymbol{I}_{E}
$$

The additional effect of the atmospheric depolarisation, $\mathbf{F}(a)$, on the analyser Eq. (D7) is

$$
\begin{aligned}
& \frac{\left\langle\mathbf{A}_{S}\right|}{T_{O} T_{S} F_{11}}=\frac{\left\langle\mathbf{M}_{S} \mathbf{R}_{\mathrm{y}} \mathbf{M}_{O}(\gamma)\right| \mathbf{F}(a)}{T_{O} T_{S} F_{11}}= \\
& =\left(\begin{array}{c}
1+\mathrm{yc}_{2 \gamma} D_{S} D_{O} \\
\mathrm{c}_{2 \gamma} D_{O}+\mathrm{y} D_{S}\left(1-\mathrm{s}_{2 \gamma}^{2} W_{O}\right) \\
\mathrm{s}_{2 \gamma}\left(D_{O}+\mathrm{yc}_{2 \gamma} D_{S} W_{O}\right) \\
-\mathrm{ys}_{2 \gamma} D_{S} Z_{O} \mathrm{~s}_{O}
\end{array} \mid\left(\begin{array}{cccc}
1 & 0 & 0 & 0 \\
0 & a & 0 & 0 \\
0 & 0 & -a & 0 \\
0 & 0 & 0 & 1-2 a
\end{array}\right)=\left(\begin{array}{c}
1+\mathrm{yc}_{2 \gamma} D_{S} D_{O} \\
a\left[\mathrm{c}_{2 \gamma} D_{O}+\mathrm{y} D_{S}\left(1-\mathrm{s}_{2 \gamma}^{2} W_{O}\right)\right] \\
-a \mathrm{~s}_{2 \gamma}\left(D_{O}+\mathrm{yc}_{2 \gamma} D_{S} W_{O}\right) \\
-(1-2 a) \mathrm{ys}_{2 \gamma} D_{S} Z_{O} \mathrm{~s}_{O}
\end{array} \mid\right.\right.
\end{aligned}
$$

Without receiver optics rotation $\mathbf{M}_{\boldsymbol{o}}\left(\gamma=0^{\circ}\right)$ we get with Eq. (D8)ff.

$$
\left\langle\mathbf{A}_{S}\right|=\left\langle\mathbf{M}_{S \mathrm{y} O}\left(0^{\circ}\right)\right| \mathbf{F}(a)=T_{\text {Syo } O}\left\langle\begin{array}{llll}
1 & a D_{S y O} & 0 & 0
\end{array}\right|
$$

\section{Appendix E: The input Stokes vector $I_{\text {in }}$}

The formulation for the most general input Stokes vector $\boldsymbol{I}_{\text {in }}$ into the analyser part $\mathbf{A}_{S}$ is

$$
\boldsymbol{I}_{\text {in }}(\gamma, a, \beta)=\mathbf{M}_{O}(\gamma) \mathbf{F}(a) \mathbf{M}_{E}(\beta) \boldsymbol{I}_{L}
$$

and assuming a rotated, partly linearly polarised laser with polarisation parameter $a_{L}$

$$
\boldsymbol{I}_{\text {in }}\left(\gamma, a, \beta, \alpha, a_{L}\right)=\mathbf{M}_{O}(\gamma) \mathbf{F}(a) \mathbf{M}_{E}(\beta) \boldsymbol{I}_{L}\left(\alpha, a_{L}\right)
$$


In the ideal case the laser has no depolarisation $\left(a_{L}=1\right)$ and is horizontally linearly polarised (see Eq. E6), and the optical elements are not rotated, which results in Eq. (E3):

$a_{L}=1, i_{L}=q_{L}=1, u_{L}=v_{L}=0, \alpha=\beta=\gamma=0 \Rightarrow$

$\boldsymbol{I}_{\text {in }}(0,0,0,0,1)=T_{O} F_{11} T_{E} I_{L}\left(1+D_{E}\right)\left|1+a D_{O} \quad D_{O}+a \quad 0 \quad 0\right\rangle$

\section{E1 Laser $I_{L}$}

We start with the Stokes vector for the laser beam with arbitrary state of polarisation and additionally rotated by angle $\alpha$ around the optical axis (see Eq. S.5.1.1)

$\left.\left.\boldsymbol{I}_{L}(\alpha)=I_{L} \mid \begin{array}{c}i_{L, \alpha} \\ q_{L, \alpha} \\ u_{L, \alpha} \\ v_{L, \alpha}\end{array}\right)=\left(\begin{array}{cccc}1 & 0 & 0 & 0 \\ 0 & \mathrm{c}_{2 \alpha} & -\mathrm{s}_{2 \alpha} & 0 \\ 0 & \mathrm{~s}_{2 \alpha} & \mathrm{c}_{2 \alpha} & 0 \\ 0 & 0 & 0 & 1\end{array}\right) I_{L} \mid \begin{array}{c}i_{L} \\ q_{L} \\ u_{L} \\ v_{L}\end{array}\right)=I_{L}\left|\begin{array}{c}i_{L} \\ \mathrm{c}_{2 \alpha} q_{L}-\mathrm{s}_{2 \alpha} u_{L} \\ \mathrm{~s}_{2 \alpha} q_{L}+\mathrm{c}_{2 \alpha} u_{L} \\ v_{L}\end{array}\right|$

The total, linear, and circular degree of polarisation (DOP, DLP, and DCP, respectively) do not change with such a rotation.

We get for a rotated, horizontally linearly polarised laser

$\boldsymbol{I}_{L}(\alpha)=I_{L}\left|1 \quad \mathrm{c}_{2 \alpha} \quad \mathrm{s}_{2 \alpha} \quad 0\right\rangle$

for a horizontally linearly polarised laser

$\boldsymbol{I}_{L}(0)=I_{L}|1 \quad 1 \quad 0 \quad 0\rangle$

and for a rotated, linearly polarised laser with polarisation parameter $a_{L}$ with $\delta_{L}=\left(1-a_{L}\right) /\left(1+a_{L}\right)$

$\left.\left.\boldsymbol{I}_{L}\left(\alpha, a_{L}\right)=\left(\begin{array}{cccc}1 & 0 & 0 & 0 \\ 0 & \mathrm{c}_{2 \alpha} & -\mathrm{s}_{2 \alpha} & 0 \\ 0 & \mathrm{~s}_{2 \alpha} & \mathrm{c}_{2 \alpha} & 0 \\ 0 & 0 & 0 & 1\end{array}\right) I_{L} \mid \begin{array}{c}1 \\ a_{L} \\ 0 \\ 0\end{array}\right)=I_{L} \mid \begin{array}{c}1 \\ \mathrm{c}_{2 \alpha} a_{L} \\ \mathrm{~s}_{2 \alpha} a_{L} \\ 0\end{array}\right)$ 
E2 I $I_{\text {in }}$ with $\mathrm{C}$ behind the emitter optics

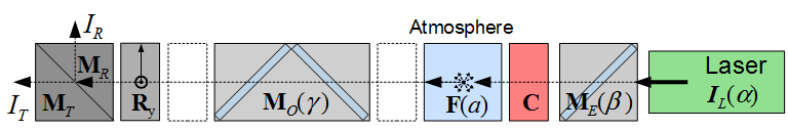

$\frac{\boldsymbol{I}_{\text {in }}(\beta, \alpha)}{T_{E} I_{L}}=\frac{\boldsymbol{I}_{E}(\beta, \alpha)}{T_{E} I_{L}}=\frac{\mathbf{M}_{E}(\beta) \boldsymbol{I}_{L}(\alpha)}{T_{E} I_{L}}=\frac{\mathbf{M}_{E}(\beta)}{T_{E} I_{L}}\left|i_{L} \quad q_{L} \quad u_{L} \quad v_{L}\right\rangle=$

$=\left|i_{i n} \quad q_{\text {in }} \quad u_{\text {in }} \quad v_{\text {in }}\right\rangle=\left|\begin{array}{llll}i_{E} & q_{E} & u_{E} & v_{E}\end{array}\right|$

Equation (E8) with input $\boldsymbol{I}_{L}$ from a rotated, linearly polarised laser Eq. (E4) and with rotated emitter optics Eq. (S.10.4.1) results in Eq. (E9).

$$
\begin{aligned}
& \frac{\boldsymbol{I}_{i n}(\beta, \alpha)}{T_{E} I_{L}}=\frac{\boldsymbol{I}_{E}(\beta, \alpha)}{T_{E} I_{L}}=\frac{\mathbf{M}_{E}(\beta)\left|\boldsymbol{I}_{L}(\alpha)\right\rangle}{T_{E} I_{L}}=\left|\begin{array}{llll}
i_{E} & q_{E} & u_{E} & v_{E}
\end{array}\right\rangle= \\
& \left.=\left(\begin{array}{cccc}
1 & \mathrm{c}_{2 \beta} D_{E} & \mathrm{~s}_{2 \beta} D_{E} & 0 \\
\mathrm{c}_{2 \beta} D_{E} & 1-\mathrm{s}_{2 \beta}{ }^{2} W_{E} & \mathrm{~s}_{2 \beta} \mathrm{c}_{2 \beta} W_{E} & -\mathrm{s}_{2 \beta} Z_{E} \mathrm{~s}_{E} \\
\mathrm{~s}_{2 \beta} D_{E} & \mathrm{~s}_{2 \beta} \mathrm{c}_{2 \beta} W_{E} & 1-\mathrm{c}_{2 \beta}{ }^{2} W_{E} & \mathrm{c}_{2 \beta} Z_{E} \mathrm{~s}_{E} \\
0 & \mathrm{~s}_{2 \beta} Z_{E} \mathrm{~s}_{E} & -\mathrm{c}_{2 \beta} Z_{E} \mathrm{~s}_{E} & Z_{E} \mathrm{c}_{E}
\end{array}\right) \mid \begin{array}{c}
i_{L} \\
\mathrm{c}_{2 \alpha} q_{L}-\mathrm{s}_{2 \alpha} u_{L} \\
\mathrm{~s}_{2 \alpha} q_{L}+\mathrm{c}_{2 \alpha} u_{L} \\
v_{L}
\end{array}\right)= \\
& =\left|\begin{array}{c}
i_{L}+D_{E}\left(\mathrm{c}_{2 \alpha-2 \beta} q_{L}-\mathrm{s}_{2 \alpha-2 \beta} u_{L}\right) \\
\mathrm{c}_{2 \beta} D_{E} i_{L}+\left(\mathrm{c}_{2 \alpha} q_{L}-\mathrm{s}_{2 \alpha} u_{L}\right)+\mathrm{s}_{2 \beta}\left[W_{E}\left(\mathrm{~s}_{2 \alpha-2 \beta} q_{L}+\mathrm{c}_{2 \alpha-2 \beta} u_{L}\right)-Z_{E} \mathrm{~s}_{E} v_{L}\right] \\
\mathrm{s}_{2 \beta} D_{E} i_{L}+\left(\mathrm{s}_{2 \alpha} q_{L}+\mathrm{c}_{2 \alpha} u_{L}\right)-\mathrm{c}_{2 \beta}\left[W_{E}\left(\mathrm{~s}_{2 \alpha-2 \beta} q_{L}+\mathrm{c}_{2 \alpha-2 \beta} u_{L}\right)-Z_{E} \mathrm{~s}_{E} v_{L}\right] \\
-Z_{E} \mathrm{~s}_{E}\left(\mathrm{~s}_{2 \alpha-2 \beta} q_{L}+\mathrm{c}_{2 \alpha-2 \beta} u_{L}\right)+Z_{E} \mathrm{c}_{E} v_{L}
\end{array}\right\rangle
\end{aligned}
$$

Special cases: Equation (E9) without rotation of the emitter optics with respect to the plane of polarisation of the laser

$$
\begin{aligned}
& \alpha=\beta \Rightarrow \\
& \frac{\boldsymbol{I}_{i n}(\alpha, \alpha)}{T_{E} I_{L}}=\frac{\boldsymbol{I}_{E}(\alpha, \alpha)}{T_{E} I_{L}}=\left|\begin{array}{l}
i_{E} \\
q_{E} \\
u_{E} \\
v_{E}
\end{array}\right|=\left|\begin{array}{c}
i_{L}+D_{E} q_{L} \\
\mathrm{c}_{2 \alpha} D_{E} i_{L}+\left(\mathrm{c}_{2 \alpha} q_{L}-\mathrm{s}_{2 \alpha} u_{L}\right)+\mathrm{s}_{2 \alpha}\left[W_{E} u_{L}-Z_{E} \mathrm{~s}_{E} v_{L}\right] \\
\mathrm{s}_{2 \alpha} D_{E} i_{L}+\left(\mathrm{s}_{2 \alpha} q_{L}+\mathrm{c}_{2 \alpha} u_{L}\right)-\mathrm{c}_{2 \alpha}\left[W_{E} u_{L}-Z_{E} \mathrm{~s}_{E} v_{L}\right] \\
-Z_{E} \mathrm{~s}_{E} u_{L}+Z_{E} \mathrm{c}_{E} v_{L}
\end{array}\right|= \\
& =\left|\begin{array}{c}
i_{L}+D_{E} q_{L} \\
\mathrm{c}_{2 \alpha}\left(D_{E} i_{L}+q_{L}\right)-\mathrm{s}_{2 \alpha} Z_{E}\left(\mathrm{c}_{E} u_{L}+\mathrm{s}_{E} v_{L}\right) \\
\mathrm{s}_{2 \alpha}\left(D_{E} i_{L}+q_{L}\right)+\mathrm{c}_{2 \alpha} Z_{E}\left(\mathrm{c}_{E} u_{L}+\mathrm{s}_{E} v_{L}\right) \\
-Z_{E}\left(\mathrm{~s}_{E} u_{L}-\mathrm{c}_{E} v_{L}\right)
\end{array}\right|
\end{aligned}
$$

Equation (E9) without laser and emitter optics rotation 


$$
\begin{aligned}
& \alpha=\beta=0 \Rightarrow \\
& \frac{\boldsymbol{I}_{i n}(0,0)}{T_{E} I_{L}}=\frac{\boldsymbol{I}_{E}(0,0)}{T_{E} I_{L}}=\frac{\mathbf{M}_{E}(0)\left|\boldsymbol{I}_{L}(0)\right\rangle}{T_{E} I_{L}}=\left|\begin{array}{c}
i_{E} \\
q_{E} \\
u_{E} \\
v_{E}
\end{array}\right\rangle=\left|\begin{array}{c}
i_{L}+D_{E} q_{L} \\
D_{E} i_{L}+q_{L} \\
Z_{E}\left(\mathrm{c}_{E} u_{L}+\mathrm{s}_{E} v_{L}\right) \\
Z_{E}\left(-\mathrm{s}_{E} u_{L}+\mathrm{c}_{E} v_{L}\right)
\end{array}\right\rangle
\end{aligned}
$$

Equation (E9) with rotated, horizontally linearly polarised laser with rotated emitter optics

$$
\begin{aligned}
& \boldsymbol{I}_{L}=I_{L} \mid \begin{array}{llll}
1 & 1 & 0 & 0
\end{array} \Rightarrow \\
& \frac{\boldsymbol{I}_{i n}(\beta, \alpha)}{T_{E} I_{L}}=\frac{\boldsymbol{I}_{E}(\beta, \alpha)}{T_{E} I_{L}}=\frac{\mathbf{M}_{E}(\beta)\left|\boldsymbol{I}_{L}(\alpha)\right\rangle}{T_{E} I_{L}}=\left|\begin{array}{c}
i_{E} \\
q_{E} \\
u_{E} \\
v_{E}
\end{array}\right|=\left|\begin{array}{c}
1+D_{E} \mathrm{c}_{2 \alpha-2 \beta} \\
\mathrm{c}_{2 \alpha}+\mathrm{c}_{2 \beta} D_{E}+\mathrm{s}_{2 \beta} W_{E} \mathrm{~s}_{2 \alpha-2 \beta} \\
\mathrm{s}_{2 \alpha}+\mathrm{s}_{2 \beta} D_{E}-\mathrm{c}_{2 \beta} W_{E} \mathrm{~s}_{2 \alpha-2 \beta} \\
-Z_{E} \mathrm{~s}_{E} \mathrm{~s}_{2 \alpha-2 \beta}
\end{array}\right\rangle
\end{aligned}
$$

Equation (E9) with rotated, linearly polarised laser without emitter optics rotation

$$
\begin{aligned}
& \alpha=\beta \wedge \boldsymbol{I}_{L}=I_{L}|1 \quad 1 \quad 0 \quad 0\rangle \Rightarrow \\
& \boldsymbol{I}_{\text {in }}(\alpha, \alpha)=T_{E} I_{L}\left|i_{E} \quad q_{E} \quad u_{E} \quad v_{E}\right\rangle=T_{E} I_{L}\left(1+D_{E}\right)\left|1 \quad c_{2 \alpha} \quad \mathrm{s}_{2 \alpha} \quad 0\right\rangle
\end{aligned}
$$

Rotated, elliptically polarised light behind the emitter optics with

$$
\boldsymbol{I}_{\text {in }}=\boldsymbol{I}_{E}=T_{E} I_{L}\left|i_{E} \quad q_{E} \quad u_{E} \quad v_{E}\right\rangle=T_{E} I_{L}\left|1 \quad b c_{2 \alpha} \quad b s_{2 \alpha} \quad v_{E}\right\rangle
$$

with the degree of polarisation $D O P_{E}=1$ and the degree of linear polarisation $D O L P_{E}=b$

$$
\begin{aligned}
& D O P_{E}=\sqrt{q_{E}^{2}+u_{E}^{2}+v_{E}^{2}}=\sqrt{b^{2}+v_{E}^{2}}=1 \Rightarrow v_{E}=\sqrt{1-b^{2}} \\
& \boldsymbol{I}_{\text {in }}=\boldsymbol{I}_{E}=T_{E} I_{L}\left|i_{E} \quad q_{E} \quad u_{E} \quad v_{E}\right\rangle=T_{E} I_{L} \mid \begin{array}{llll}
1 & b \mathrm{c}_{2 \alpha} & b \mathrm{~s}_{2 \alpha} & \left.\sqrt{1-b^{2}}\right\rangle
\end{array}
\end{aligned}
$$

Rotated, linearly polarised laser with linear polarisation parameter $a_{L}$ with rotated emitter optics: laser Stokes vector Eq. (E7) and rotated diattenuator Eq. (S.10.4.1) 


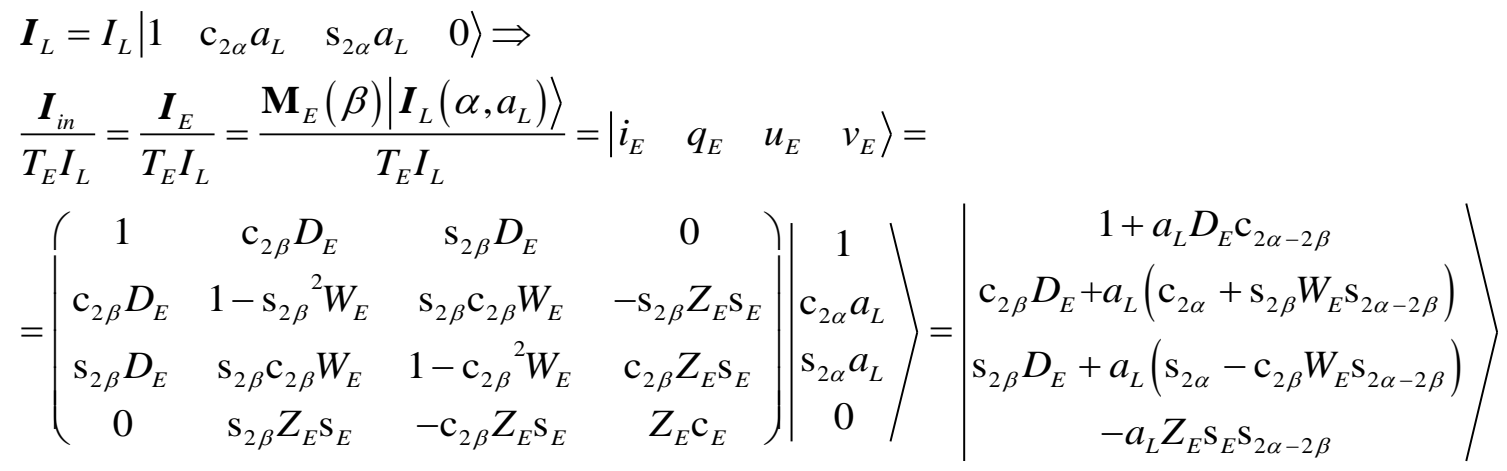

\section{E3 I $I_{\text {in }}$ with $\mathrm{C}$ before the receiver optics}

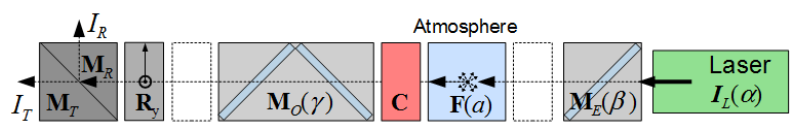

General input Stokes $\boldsymbol{I}_{\text {in }}$ vector with atmospheric backscatter.

$$
\boldsymbol{I}_{S}=\eta_{S} \mathbf{M}_{S} \mathbf{R}_{\mathrm{y}} \mathbf{M}_{O} \mathbf{C} \mathbf{F} \boldsymbol{I}_{E} \Rightarrow \boldsymbol{I}_{\text {in }}=\mathbf{F} \boldsymbol{I}_{E}
$$

With atmospheric depolarisation from Eq. (S.3.1) and an emitter beam $\boldsymbol{I}_{E}$ from Sect. E2:

$$
\boldsymbol{I}_{\text {in }}(a)=\left|\mathbf{F}(a) \boldsymbol{I}_{E}\right\rangle=F_{11} T_{E} I_{L}\left|i_{E} \quad a q_{E} \quad-a u_{E} \quad(1-2 a) v_{E}\right\rangle
$$

Special cases: Equation (E19) becomes Eq. (E20) with a rotated linearly polarised laser with linear polarisation parameter $a_{L}$, with rotated emitter optics, and atmospheric backscatter, i.e. Eq. (E17). Note that without laser depolarisation $a_{L}=1$.

$$
\begin{aligned}
& \frac{\boldsymbol{I}_{\text {in }}\left(a, \beta, \alpha, a_{L}\right)}{F_{11} T_{E} I_{L}}=\frac{\mathbf{F}(a)\left|\mathbf{M}_{E}(\beta) \boldsymbol{I}_{L}\left(\alpha, a_{L}\right)\right\rangle}{F_{11} T_{E} I_{L}}= \\
& =\left|\begin{array}{l}
i_{i n} \\
q_{\text {in }} \\
u_{\text {in }} \\
v_{\text {in }}
\end{array}\right|=\left|\begin{array}{c}
i_{E} \\
a q_{E} \\
-a u_{E} \\
(1-2 a) v_{E}
\end{array}\right|=\left|\begin{array}{c}
1+a_{L} D_{E} \mathrm{c}_{2 \alpha-2 \beta} \\
a\left[\mathrm{c}_{2 \beta} D_{E}+a_{L}\left(\mathrm{c}_{2 \alpha}+\mathrm{s}_{2 \beta} W_{E} \mathrm{~s}_{2 \alpha-2 \beta}\right)\right] \\
-a\left[\mathrm{~s}_{2 \beta} D_{E}+a_{L}\left(\mathrm{~s}_{2 \alpha}-\mathrm{c}_{2 \beta} W_{E} \mathrm{~s}_{2 \alpha-2 \beta}\right)\right] \\
-(1-2 a) a_{L} Z_{E} \mathrm{~s}_{E} \mathrm{~s}_{2 \alpha-2 \beta}
\end{array}\right\rangle
\end{aligned}
$$

Equation (E20) without rotation errors becomes Eq. (E21), and additionally without laser depolarisation, i.e. $a_{L}=1$, Eq. (E22).

$$
\begin{aligned}
& \alpha=\beta=0 \Rightarrow \\
& \frac{\boldsymbol{I}_{\text {in }}\left(a, 0,0, a_{L}\right)}{F_{11} T_{E} I_{L}}=\frac{\mathbf{F}(a)\left|\mathbf{M}_{E}(0) \boldsymbol{I}_{L}\left(0, a_{L}\right)\right\rangle}{F_{11} T_{E} I_{L}}=\left|1+a_{L} D_{E} \quad a D_{E}+a a_{L} \quad 0 \quad 0\right\rangle
\end{aligned}
$$




$$
\boldsymbol{I}_{\text {in }}(a, 0,0,0)=F_{11} T_{E} I_{L}\left(1+D_{E}\right)|1 \quad a \quad 0 \quad 0\rangle
$$

Equation (E20) without emitter optics becomes Eq. (E23).

$$
\begin{aligned}
& {\left[D_{E}=0 \Rightarrow Z_{E}=1, \mathrm{~s}_{E}=0 \Rightarrow \mathrm{c}_{E}=1 \Rightarrow W_{E}=0\right] \Rightarrow} \\
& \frac{\boldsymbol{I}_{i n}\left(a,, \alpha, a_{L}\right)}{F_{11} I_{L}}=\frac{\mathbf{F}(a)\left|\boldsymbol{I}_{L}\left(\alpha, a_{L}\right)\right\rangle}{F_{11} I_{L}}=\left|\begin{array}{llll}
1 & a a_{L} \mathrm{c}_{2 \alpha} & -a a_{L} \mathrm{~s}_{2 \alpha} & 0
\end{array}\right\rangle
\end{aligned}
$$

Note it is impossible to combine $a^{\prime}=a a_{L}$ if emitter optics $\mathbf{M}_{E}$ with diattenuation parameter $D_{E} \neq 0$ or retardation (i.e. $Z_{E}$ $\neq 0$ and $\mathbf{s}_{E} \neq 0$ ) are between the laser and the atmosphere $\mathbf{F}$, even if there are no angular misalignments $\alpha$ and $\beta$ in the emitter, which means that the atmospheric depolarisation cannot be retrieved without detailed knowledge of the emitter optics parameters and alignment errors.

Equation (E20) without emitter optics $\mathbf{M}_{E}$ and without laser depolarisation becomes Eq. (E24).

$$
\begin{aligned}
& a_{L}=1,\left[D_{E}=0 \Rightarrow Z_{E}=1, \mathrm{~s}_{E}=0 \Rightarrow \mathrm{c}_{E}=1 \Rightarrow W_{E}=0\right] \Rightarrow \\
& \left.\frac{\boldsymbol{I}_{\text {in }}(a,, \alpha)}{I_{\text {in }}}=\frac{\mathbf{F}(a)\left|\boldsymbol{I}_{L}(\alpha)\right\rangle}{F_{11} I_{L}}=\mid \begin{array}{llll}
1 & a \mathrm{c}_{2 \alpha} & -a \mathrm{~s}_{2 \alpha} & 0
\end{array}\right)
\end{aligned}
$$

- Equation (E19) with $\boldsymbol{I}_{E}$ from Eq. (E14), i.e. with rotated, elliptically polarised light behind the emitter optics

$$
\begin{aligned}
& \frac{\boldsymbol{I}_{\text {in }}(a, b, \alpha)}{I_{\text {in }}}=\frac{\mathbf{F}(a) \boldsymbol{I}_{E}}{F_{11} T_{E} I_{L}}=\left|\begin{array}{llll}
i_{E} & a q_{E} & -a u_{E} & (1-2 a) v_{E}
\end{array}\right\rangle= \\
& =\left|\begin{array}{llll}
1 & a b \mathrm{c}_{2 \alpha} & -a b \mathrm{~s}_{2 \alpha} & (1-2 a) \sqrt{1-b^{2}}
\end{array}\right\rangle
\end{aligned}
$$

Including the calibrator rotation $\mathrm{R}(\varepsilon)$ in $I_{\text {in }}$ in Eq. (E19) with Eq. (S.10.15.1) gives Eq. (E26), and with an elliptically polarised laser of Eq. (E16) we get Eq. (E27), which results without emitter optics and horizontally linearly polarised laser light $(b=1)$ in Eq. (E28).

$$
\begin{aligned}
& \frac{\boldsymbol{I}_{i n, \varepsilon}(\varepsilon, \mathrm{h}, a)}{I_{i n}}=\frac{\left|\mathbf{R}(\varepsilon) \mathbf{M}_{\mathrm{h}} \mathbf{F}(a) \boldsymbol{I}_{E}\right\rangle}{T_{r o t} F_{11} T_{E} I_{L}}= \\
& =\left|\begin{array}{l}
i_{i n, \varepsilon} \\
q_{i n, \varepsilon} \\
u_{i n, \varepsilon} \\
v_{i n, \varepsilon}
\end{array}\right\rangle=\left(\begin{array}{cccc}
1 & 0 & 0 & 0 \\
0 & \mathrm{c}_{2 \varepsilon} & -\mathrm{hs}_{2 \varepsilon} & 0 \\
0 & \mathrm{~s}_{2 \varepsilon} & \mathrm{hc}_{2 \varepsilon} & 0 \\
0 & 0 & 0 & \mathrm{~h}
\end{array}\right)\left|\begin{array}{c}
i_{E} \\
a q_{E} \\
-a u_{E} \\
(1-2 a) v_{E}
\end{array}\right|=\left|\begin{array}{c}
i_{E} \\
a\left(q_{E} \mathrm{c}_{2 \varepsilon}+\mathrm{h} u_{E} \mathrm{~s}_{2 \varepsilon}\right) \\
a\left(q_{E} \mathrm{~s}_{2 \varepsilon}-\mathrm{h} u_{E} \mathrm{c}_{2 \varepsilon}\right) \\
(1-2 a) \mathrm{h} v_{E}
\end{array}\right\rangle
\end{aligned}
$$




$$
\begin{aligned}
& \boldsymbol{I}_{E}=T_{E} I_{L}\left|i_{E} \quad q_{E} \quad u_{E} \quad v_{E}\right\rangle=T_{E} I_{L}\left|\begin{array}{llll}
1 & b \mathrm{c}_{2 \alpha} & b \mathrm{~s}_{2 \alpha} & \sqrt{1-b^{2}}
\end{array}\right\rangle \Rightarrow \\
& \frac{\boldsymbol{I}_{i n, \varepsilon}(\varepsilon, \mathrm{h}, a, \alpha, b)}{I_{i n}}=\frac{\left|\mathbf{R}(\varepsilon) \mathbf{M}_{\mathrm{h}} \mathbf{F}(a) \boldsymbol{I}_{E}(\alpha, b)\right\rangle}{T_{r o t} F_{11} T_{E} I_{L}}= \\
& =\left|\begin{array}{lllll}
i_{i n, \varepsilon} & q_{i n, \varepsilon} & u_{i n, \varepsilon} & v_{i n, \varepsilon}
\end{array}\right\rangle=\left|\begin{array}{llll}
1 & a b \mathrm{c}_{2 \varepsilon-\mathrm{h} 2 \alpha} & a b \mathrm{~s}_{2 \varepsilon+\mathrm{h} 2 \alpha} & (1-2 a) \mathrm{h} \sqrt{1-b^{2}}
\end{array}\right\rangle
\end{aligned}
$$

$\mathbf{M}_{E}=$ idendity, $b=1 \Rightarrow$

$\frac{\boldsymbol{I}_{i n, \varepsilon}(\varepsilon, \mathrm{h}, a, \alpha, b)}{I_{i n}}=\frac{\left|\mathbf{R}(\varepsilon) \mathbf{M}_{\mathrm{h}} \mathbf{F}(a) \boldsymbol{I}_{L}(\alpha, b)\right\rangle}{T_{r o t} F_{11} I_{L}}=$

$$
=\left|\begin{array}{llll}
i_{i n, \varepsilon} & q_{i n, \varepsilon} & u_{i n, \varepsilon} & v_{i n, \varepsilon}
\end{array}\right\rangle=\left|\begin{array}{llll}
1 & a \mathrm{c}_{2 \varepsilon-\mathrm{h} 2 \alpha} & a \mathrm{~s}_{2 \varepsilon+\mathrm{h} 2 \alpha} & 0
\end{array}\right\rangle
$$

\section{E4 I $I_{\text {in }}$ with $\mathrm{C}$ before the polarising beam splitter}

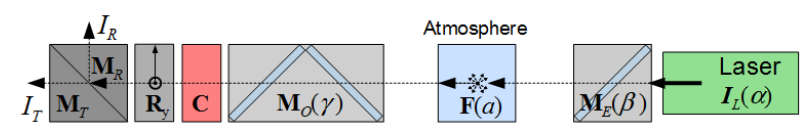

General input vector $\boldsymbol{I}_{\text {in }}$ with atmospheric backscatter and emitter, and receiver optics.

$$
\boldsymbol{I}_{S}=\eta_{S} \mathbf{M}_{S} \mathbf{R}_{\mathrm{y}} \mathbf{C} \mathbf{M}_{O} \mathbf{F} \mathbf{M}_{E} \boldsymbol{I}_{L} \Rightarrow \boldsymbol{I}_{\text {in }}=\mathbf{M}_{O} \mathbf{F} \boldsymbol{I}_{E}
$$

The most complex case for the input Stokes vector $\boldsymbol{I}_{\text {in }}$ is when the calibrator is placed before the polarising beam splitter because here we have to multiply several matrices. All other cases can be derived from this case by neglecting the appropriate parameters (see 0). The emitted beam Stokes vector $\boldsymbol{I}_{E}$ from Sect. E2 has to be multiplied with the atmospheric backscatter matrix $\mathbf{F}$ (Eq. S.3.1) and the receiver optics matrix $\mathbf{M}_{O}$, the latter expressed as a rotated diattenuator (see Eq. E32). In general the emitter optics and the laser polarisation $\boldsymbol{I}_{L}$ are rotated as in Eq. (E30), which is not mentioned explicitly when needless.

$\boldsymbol{I}_{E}(\beta, \alpha)=\mathbf{M}_{E}(\beta)\left|\boldsymbol{I}_{L}(\alpha)\right\rangle=T_{E} I_{L}\left|i_{E}(\beta, \alpha) \quad q_{E}(\beta, \alpha) \quad u_{E}(\beta, \alpha) \quad v_{E}(\beta, \alpha)\right\rangle$ 


$$
\begin{aligned}
& \frac{\boldsymbol{I}_{i n}(\gamma, a,,)}{T_{i n} I_{L}}=\frac{\mathbf{M}_{O}(\gamma)\left|\mathbf{F}(a) \mathbf{M}_{E} \boldsymbol{I}_{L}\right\rangle}{T_{O} F_{11} T_{E} I_{L}}=\frac{\mathbf{M}_{O}(\gamma)\left|\mathbf{F}(a) \boldsymbol{I}_{E}\right\rangle}{T_{O} F_{11} T_{E} I_{L}}=\left|i_{\text {in }} \quad q_{\text {in }} \quad u_{\text {in }} \quad v_{i n}\right\rangle= \\
& \left.=\left(\begin{array}{cccc}
1 & \mathrm{c}_{2 \gamma} D_{O} & \mathrm{~s}_{2 \gamma} D_{O} & 0 \\
\mathrm{c}_{2 \gamma} D_{O} & 1-\mathrm{s}_{2 \gamma}^{2} W_{O} & \mathrm{~s}_{2 \gamma} \mathrm{c}_{2 \gamma} W_{O} & -\mathrm{s}_{2 \gamma} Z_{O} \mathrm{~s}_{O} \\
\mathrm{~s}_{2 \gamma} D_{O} & \mathrm{~s}_{2 \gamma} \mathrm{c}_{2 \gamma} W_{O} & 1-\mathrm{c}_{2 \gamma}^{2} W_{O} & \mathrm{c}_{2 \gamma} Z_{O} \mathrm{~s}_{O} \\
0 & \mathrm{~s}_{2 \gamma} Z_{O} \mathrm{~s}_{O} & -\mathrm{c}_{2 \gamma} Z_{O} \mathrm{~s}_{O} & Z_{O} \mathrm{c}_{O}
\end{array}\right) \mid \begin{array}{c}
i_{E} \\
a q_{E} \\
-a u_{E} \\
(1-2 a) v_{E}
\end{array}\right)= \\
& =\left|\begin{array}{c}
i_{E}+D_{O} a\left(\mathrm{c}_{2 \gamma} q_{E}-\mathrm{s}_{2 \gamma} u_{E}\right) \\
\mathrm{c}_{2 \gamma} D_{O} i_{E}+a q_{E}-\mathrm{s}_{2 \gamma}\left[W_{O} a\left(\mathrm{~s}_{2 \gamma} q_{E}+\mathrm{c}_{2 \gamma} u_{E}\right)+Z_{O} \mathrm{~s}_{O}(1-2 a) v_{E}\right] \\
\mathrm{s}_{2 \gamma} D_{O} i_{E}-a u_{E}+\mathrm{c}_{2 \gamma}\left[W_{O} a\left(\mathrm{~s}_{2 \gamma} q_{E}+\mathrm{c}_{2 \gamma} u_{E}\right)+Z_{O} \mathrm{~s}_{O}(1-2 a) v_{E}\right] \\
Z_{O} \mathrm{~s}_{O} a\left(\mathrm{~s}_{2 \gamma} q_{E}+\mathrm{c}_{2 \gamma} u_{E}\right)+Z_{O} \mathrm{c}_{O}(1-2 a) v_{E}
\end{array}\right\rangle
\end{aligned}
$$

Special cases: From Eq. (E31) without receiver optics rotation $\gamma$ we get Eq. (E32).

$$
\begin{aligned}
& \gamma=0 \Rightarrow \\
& \left.\frac{\boldsymbol{I}_{i n}(0, a,)}{T_{i n} I_{L}}=\frac{\mathbf{M}_{O}(0)\left|\mathbf{F}(a) \mathbf{M}_{E} \boldsymbol{I}_{L}\right\rangle}{T_{O} F_{11} T_{E} I_{L}}=\left|\begin{array}{l}
i_{\text {in }} \quad q_{i n} \quad u_{i n} \quad v_{i n}
\end{array}\right\rangle=\mid \begin{array}{c}
i_{E}+a D_{O} q_{E} \\
D_{O} i_{E}+a q_{E} \\
Z_{O}\left[\begin{array}{c}
-\mathrm{c}_{O} a u_{E}+\mathrm{s}_{O}(1-2 a) v_{E}
\end{array}\right] \\
Z_{O}\left[\mathrm{~s}_{O} a u_{E}+\mathrm{c}_{O}(1-2 a) v_{E}\right.
\end{array}\right]
\end{aligned}
$$

With linearly polarised laser $\boldsymbol{I}_{L}$ with polarisation parameter $a_{L}$, with emitter optics $\mathbf{M}_{E}$, atmosphere $\mathbf{F}$, and receiver optics $\mathbf{M}_{O}$, and with Eqs. (E32) and (E20) we get Eq. (E33).

$$
\begin{aligned}
& i_{L}=q_{L}=1, u_{L}=v_{L}=0 \Rightarrow \\
& \frac{\boldsymbol{I}_{i n}\left(\gamma, a, \beta, \alpha, a_{L}\right)}{T_{i n} I_{L}}=\frac{\mathbf{M}_{O}(\gamma)\left|\mathbf{F}(a) \mathbf{M}_{E}(\beta) \boldsymbol{I}_{L}\left(\alpha, a_{L}\right)\right\rangle}{T_{O} F_{11} T_{E} I_{L}}= \\
& =\left(\begin{array}{cccc}
1 & \mathrm{c}_{2 \gamma} D_{O} & \mathrm{~s}_{2 \gamma} D_{O} & 0 \\
\mathrm{c}_{2 \gamma} D_{O} & 1-\mathrm{s}_{2 \gamma}^{2} W_{O} & \mathrm{~s}_{2 \gamma} \mathrm{c}_{2 \gamma} W_{O} & -\mathrm{s}_{2 \gamma} Z_{O} \mathrm{~s}_{O} \\
\mathrm{~s}_{2 \gamma} D_{O} & \mathrm{~s}_{2 \gamma} \mathrm{c}_{2 \gamma} W_{O} & 1-\mathrm{c}_{2 \gamma}^{2} W_{O} & \mathrm{c}_{2 \gamma} Z_{O} \mathrm{~s}_{O} \\
0 & \mathrm{~s}_{2 \gamma} Z_{O} \mathrm{~s}_{O} & -\mathrm{c}_{2 \gamma} Z_{O} \mathrm{~s}_{O} & Z_{O} \mathrm{c}_{O}
\end{array}\right) \mid \begin{array}{c}
1+a_{L} D_{E} \mathrm{c}_{2 \alpha-2 \beta} \\
a\left[\begin{array}{c}
\left.\mathrm{c}_{2 \beta} D_{E}+a_{L}\left(\mathrm{c}_{2 \alpha}+\mathrm{s}_{2 \beta} W_{E} \mathrm{~s}_{2 \alpha-2 \beta}\right)\right] \\
-a\left[\mathrm{~s}_{2 \beta} D_{E}+a_{L}\left(\mathrm{~s}_{2 \alpha}-\mathrm{c}_{2 \beta} W_{E} \mathrm{~s}_{2 \alpha-2 \beta}\right)\right] \\
-(1-2 a) a_{L} Z_{E} \mathrm{~s}_{E} \mathrm{~s}_{2 \alpha-2 \beta}
\end{array}\right)
\end{array}
\end{aligned}
$$

Equation (E33) with rotated, linearly polarised laser without laser depolarisation $\left(a_{L}=1\right)$ and rotated emitter optics (Eq. E20) the input Stokes vector becomes explicitly 


$$
\begin{aligned}
& a_{L}=1, i_{L}=q_{L}=1, u_{L}=v_{L}=0, \gamma=0 \Rightarrow \\
& \frac{\boldsymbol{I}_{i n}}{T_{i n} I_{L}}=\frac{\mathbf{M}_{O}(0)\left|\mathbf{F}(a) \mathbf{M}_{E}(\beta) \boldsymbol{I}_{L}(\alpha)\right\rangle}{T_{O} F_{11} T_{E} I_{L}}= \\
& \left(1+D_{E} \mathrm{c}_{2 \alpha-2 \beta}\right)+a D_{O}\left(\mathrm{c}_{2 \alpha}+\mathrm{c}_{2 \beta} D_{E}+\mathrm{s}_{2 \beta} W_{E} \mathrm{~s}_{2 \alpha-2 \beta}\right) \\
& D_{O}\left(1+D_{E} \mathrm{c}_{2 \alpha-2 \beta}\right)+a\left(\mathrm{c}_{2 \alpha}+\mathrm{c}_{2 \beta} D_{E}+\mathrm{s}_{2 \beta} W_{E} \mathrm{~s}_{2 \alpha-2 \beta}\right) \\
& \left.=\mid \begin{array}{c}
\left.\left.\left.\mathrm{c}_{2 \beta} W_{E} \mathrm{~s}_{2 \alpha-2 \beta}\right)-2 \mathrm{~s}_{O} Z_{E} \mathrm{~s}_{E} \mathrm{~s}_{2 \alpha-2 \beta}\right]\right\} \\
-Z_{O}\left\{\mathrm{~s}_{O} Z_{E} \mathrm{~s}_{E} \mathrm{~s}_{2 \alpha-2 \beta}+a\left[\mathrm{c}_{O}\left(\mathrm{~s}_{2 \alpha}+\mathrm{s}_{2 \beta} D_{E}-\mathrm{c}_{2 \beta} W_{E}\right)\right.\right. \\
-Z_{O}\left\{\mathrm{c}_{O} Z_{E} \mathrm{~s}_{E} \mathrm{~s}_{2 \alpha-2 \beta}-a\left[\mathrm{~s}_{O}\left(\mathrm{~s}_{2 \alpha}+\mathrm{s}_{2 \beta} D_{E}-\mathrm{c}_{2 \beta} W_{E} \mathrm{~s}_{2 \alpha-2 \beta}\right)+2 \mathrm{c}_{O} Z_{E} \mathrm{~s}_{E} \mathrm{~s}_{2 \alpha-2 \beta}\right]\right\}
\end{array}\right)
\end{aligned}
$$

Equation (E34) with laser polarisation and emitter optics aligned

$$
\begin{aligned}
& a_{L}=1, i_{L}=q_{L}=1, u_{L}=v_{L}=0, \gamma=0, \beta=\alpha \Rightarrow \\
& \frac{\boldsymbol{I}_{\text {in }}}{T_{\text {in }} I_{L}}=\frac{\mathbf{M}_{O}(0)\left|\mathbf{F}(a) \mathbf{M}_{E}(\alpha) \boldsymbol{I}_{L}(\alpha)\right\rangle}{T_{O} F_{11} T_{E} I_{L}}=\left(1+D_{E}\right)\left|\begin{array}{c}
1+a D_{O} \mathrm{c}_{2 \alpha} \\
D_{O}+a \mathrm{c}_{2 \alpha} \\
-Z_{O} a \mathrm{c}_{O} \mathrm{~s}_{2 \alpha} \\
+Z_{O} a \mathrm{~s}_{O} \mathrm{~s}_{2 \alpha}
\end{array}\right|
\end{aligned}
$$

and without any optics and laser rotation

$$
\begin{aligned}
& a_{L}=1, i_{L}=q_{L}=1, u_{L}=v_{L}=0, \alpha=\beta=\gamma=0 \Rightarrow \\
& \frac{\boldsymbol{I}_{\text {in }}(0,0,0,0,1)}{T_{\text {in }} I_{L}}=\frac{\mathbf{M}_{O}(0)\left|\mathbf{F}(a) \mathbf{M}_{E}(0) \boldsymbol{I}_{L}(0)\right\rangle}{T_{O} F_{11} T_{E} I_{L}}=\left(1+D_{E}\right)\left|1+a D_{O} \quad D_{O}+a \quad 0 \quad 0\right\rangle
\end{aligned}
$$

Equation (E.33) without emitter optics $\mathbf{M}_{E}$

$$
\begin{aligned}
& D_{E}=0, \mathrm{~s}_{E}=0, W_{E}=0, a^{\prime}=a a_{L} \Rightarrow \\
& \frac{\boldsymbol{I}_{i n}\left(\gamma, a, 0, \alpha, a_{L}\right)}{T_{i n} I_{L}}=\frac{\mathbf{M}_{O}(\gamma) \mathbf{F}(a) \boldsymbol{I}_{L}\left(\alpha, a_{L}\right)}{T_{O} F_{11} I_{L}}=\left[\begin{array}{c}
1+\mathrm{c}_{2 \gamma+2 \alpha} a^{\prime} D_{O} \\
\mathrm{c}_{2 \gamma} D_{O}+a^{\prime}\left[\mathrm{c}_{2 \alpha}-\mathrm{s}_{2 \gamma} \mathrm{s}_{2 \gamma+2 \alpha} W_{O}\right] \\
\mathrm{s}_{2 \gamma} D_{O}-a^{\prime}\left[\mathrm{s}_{2 \alpha}-\mathrm{c}_{2 \gamma} \mathrm{s}_{2 \gamma+2 \alpha} W_{O}\right] \\
\mathrm{s}_{2 \gamma+2 \alpha} a^{\prime} Z_{O} \mathrm{~s}_{O}
\end{array}\right)
\end{aligned}
$$

No emitter optics $\mathbf{M}_{E}$ and no receiver optics rotation

with $\gamma=0, T_{E}=1, D_{E}=0, \mathrm{~s}_{E}=0, W_{E}=0, a^{\prime}=a a_{L} \Rightarrow$

$$
\begin{aligned}
& \frac{\boldsymbol{I}_{\text {in }}\left(0, a, 0, \alpha, a_{L}\right)}{T_{i n} I_{L}}=\frac{\mathbf{M}_{O}(0) \mathbf{F}(a) \boldsymbol{I}_{L}\left(\alpha, a_{L}\right)}{T_{O} F_{11} I_{L}}= \\
& =\left|\begin{array}{llll}
1+\mathrm{c}_{2 \alpha} a^{\prime} D_{O} & D_{O}+\mathrm{c}_{2 \alpha} a^{\prime} & -\mathrm{s}_{2 \alpha} a^{\prime} Z_{O} \mathrm{c}_{O} & \mathrm{~s}_{2 \alpha} a^{\prime} Z_{O} \mathrm{~s}_{O}
\end{array}\right\rangle
\end{aligned}
$$


The latter and no laser rotation

with $\alpha=0, \gamma=0, T_{E}=1, D_{E}=0, \mathrm{~s}_{E}=0, W_{E}=0, a^{\prime}=a a_{L} \Rightarrow$

$$
\frac{\boldsymbol{I}_{\text {in }}\left(0, a, 0,0, a_{L}\right)}{T_{\text {in }} I_{L}}=\frac{\mathbf{M}_{O}(0) \mathbf{F}(a) \boldsymbol{I}_{L}\left(0, a_{L}\right)}{T_{O} F_{11} I_{L}}=\left|1+a^{\prime} D_{O} \quad D_{O}+a^{\prime} \quad 0 \quad 0\right\rangle
$$

E5 $I_{\text {in }}$ with $\mathrm{C}$ amidst the receiving optics

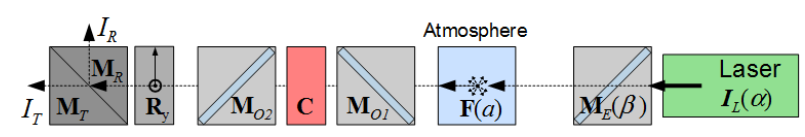

In the case that there is polarising or/and retarding optics before $\left(\mathbf{M}_{O 1}\right)$ and after $\left(\mathbf{M}_{O 2}\right)$ the calibrator as in Eq. (E40), the basic equations can be constructed by using the analyser matrix $\mathbf{A}_{S}$ from Sect. D2 and the input Stokes vectors $\boldsymbol{I}_{\text {in }}$ from Sect. E4.

$\boldsymbol{I}_{S}=\eta_{S} \mathbf{M}_{S} \mathbf{R}_{\mathrm{y}} \mathbf{M}_{O 2} \mathbf{C} \mathbf{M}_{O 1} \mathbf{F} \boldsymbol{I}_{E} \Rightarrow \mathbf{A}_{S}=\mathbf{M}_{S} \mathbf{R}_{\mathrm{y}} \mathbf{M}_{O 2}$ and $\boldsymbol{I}_{\text {in }}=\mathbf{M}_{O 1} \mathbf{F} \boldsymbol{I}_{E}$ 


\title{
The Supplement related to this article is available online at doi:10.5194/amt-9-4181-2016-supplement.
}

\begin{abstract}
Acknowledgements. The financial support for EARLINET in the ACTRIS Research Infrastructure Project by the European Union's Horizon 2020 research and innovation programme under grant agreement no. 654169 and previously under grant agreement no. 262254 in the 7th Framework Programme (FP7/2007-2013) is gratefully acknowledged.
\end{abstract}

Edited by: A. Ansmann

Reviewed by: three anonymous referees

\section{References}

Alvarez, J. M., Vaughan, M. A., Hostetler, C. A., Hunt, W. H., and Winker, D. M.: Calibration Technique for Polarization-Sensitive Lidars, J. Atmos. Ocean. Tech., 23, 683-699, 2006.

Anderson, R.: Polarization and atmospheric backscatter coefficient measurements, Appl. Optics, 28, 865-874, 1989.

Ansmann, A. and Müller, D.: Lidar and Atmospheric Aerosol Particles, in: Lidar, edited by: Weitkamp, C., Springer New York, 105-141, 2005.

Ansmann, A., Tesche, M., Seifert, P., Groß, S., Freudenthaler, V., Apituley, A., Wilson, K. M., Serikov, I., Linné, H., Heinold, B., Hiebsch, A., Schnell, F., Schmidt, J., Mattis, I., Wandinger, U., and Wiegner, M.: Ash and fine-mode particle mass profiles from EARLINET-AERONET observations over central Europe after the eruptions of the Eyjafjallajökull volcano in 2010, J. Geophys. Res., 116, D00U02, doi:10.1029/2010JD015567, 2011.

Azzam, R. M. A.: Ellipsometry in: Handbook of Optics, Volume I, 3rd Edn., chap. 16, McGraw-Hill, 2009.

Behrendt, A. and Nakamura, T.: Calculation of the calibration constantof polarization lidar and its dependency on atmospheric temperature, Opt. Express, 10, 805-817, 2002.

Bennett, J. M.: Polarization in: Handbook of Optics, Volume I, 3rd Edn., chap. 12, McGraw-Hill, 2009 a.

Bennett, J. M.: Polarizers in: Handbook of Optics, Volume I, 3rd Edn., chap. 13, McGraw-Hill, $2009 \mathrm{~b}$

Beyerle, G.: Untersuchungen stratosphärischer Aerosole vulkanischen Ursprungs und polarer stratosphärischer Wolken mit einem Mehrwellen-Lidar auf Spitzbergen $\left(79^{\circ} \mathrm{N}, 12^{\circ} \mathrm{E}\right)$, Berichte zur Polarforschung (Reports on Polar Research), 138, 130 pp, hdl:10013/epic.10139.d001, 1994.

Biele, J., Beyerle, G., and Baumgarten, G.: Polarization Lidar: Correction of instrumental effects, Opt. Express, 7, 427435, 2000. 
Böckmann, C. and Osterloh, L.: Runge-Kutta type regularization method for inversion of spheroidal particle distribution from limited optical data, Inverse Problems in Science and Engineering, 22, 150-165, 2014.

Borovoi, A., Konoshonkin, A. and Kustova, N.: Backscatter ratios for arbitrary oriented hexagonal ice crystals of cirrus clouds, Opt. Lett., 39, 5788-5791, 2014.

Boucher, O., Randall, D., Artaxo, P., Bretherton, C., Feingold, G., Forster, P., Kerminen, V.-M., Kondo, Y., Liao, H., Lohmann, U., Rasch, P., Satheesh, S., Sherwood, S., Stevens, B., and Zhang, X.: in: Clouds and Aerosols, Cambridge University Press, Cambridge, United Kingdom and New York, NY, USA, 571-658, 2013.

Bravo-Aranda, J. A., Belegante, L., Freudenthaler, V., Alados-Arboledas, A., Nicolae, D., Granados-Muñoz, M. J., Guerrero- Rascado, J. L., Amodeo, A., D’Amico, G., Engelmann, R., Pappalardo, G., Kokkalis, P., Mamouri, R., Papayannis, A., Navas-Guzm F., Olmo, F. J., Wandinger, U., and Haeffelin, M.: Assessment of lidar depolarization uncertainty by means of a polarimetric lidar simulator, Atmos. Meas. Tech. Discuss.,

doi:10.5194/amt-2015-339, in review, 2016.

Bravo-Aranda, J. A., Navas-Guzmán, F., Guerrero-Rascado, J. L., Pérez-Ramírez, D., Granados-Muñoz, M. J., and Alados-Arboledas, L.: Analysis of lidar depolarization calibration procedure and application to the atmospheric aerosol characterization, Int. J. Remote Sens., 34, 3543-3560, 2013.

Breckinridge, J. B., Lam, W. S. T., and Chipman, R. A.: Polarization Aberrations in Astronomical Telescopes: The Point Spread Function, Publ. Astron. Soc. Pacific, 127, 445-468, 2015.

Burton, S. P., Hair, J. W., Kahnert, M., Ferrare, R. A., Hostetler, C. A., Cook, A. L., Harper, D. B., Berkoff, T. A., Seaman, S. T., Collins, J. E., Fenn, M. A., and Rogers, R. R.: Observations of the spectral dependence of linear particle depolarization ratio of aerosols using NASA Langley airborne High Spectral Resolution Lidar, Atmos. Chem. Phys., 15, 13453-13473, doi:10.5194/acp-15-13453-2015, 2015.

Burton, S. P., Vaughan, M. A., Ferrare, R. A., and Hostetler, C. A.: Separating mixtures of aerosol types in airborne High Spectral Resolution Lidar data, Atmos. Meas. Tech., 7, 419-436, doi:10.5194/amt-7-419-2014, 2014.

Cairo, F., Donfrancesco, G. D., Adriani, A., Pulvirenti, L., and Fierli, F.: Comparison of Various Linear Depolarization Parameters Measured by Lidar, Appl. Optics, 38, 4425-4432, 1999.

Cao, X., Roy, G., and Bernier, R.: Lidar polarization discrimination of bioaerosols, in: Proc. SPIE 7672, Polarization: Measurement, Analysis, and Remote Sensing IX, 76720P, doi:10.1117/12.849649, 26 April 2010.

Chipman, R. A.: Mueller matrices in: Handbook of Optics, Volume I, 3rd Edn., chap. 14, McGraw-Hill, 2009 b.

Chipman, R. A.: Polarimetry in: Handbook of Optics, Volume I, 3rd Edn., chap. 15, McGraw-Hill, 2009 a.

Clark, N. and Breckinridge, J. B.: Polarization compensation of Fresnel aberrations in telescopes, in: Proc. SPIE 8146, UV/Optical/IR Space Telescopes and Instruments: Innovative Technologies and Concepts V, 81460O, doi:10.1117/12.896638, 14 September 2011.

Clarke, D.: Stellar Polarimetry, Wiley-VCH Verlag GmbH \& Co. KGaA, 2009.

David, G., Miffre, A., Thomas, B., and Rairoux, P.: Sensitive and accurate dual-wavelength UV-VIS polarization detector for optical remote sensing of tropospheric aerosols, Appl. Phys. B, 108, 197-216, 2012.

David, G., Thomas, B., Coillet, E., Miffre, A., and Rairoux, P.: Polarization-resolved exact light backscattering by an ensemble of particles in air, Opt. Express, 21, 18624-18639, 2013. 
Del Guasta, M., Vallar, E., Riviere, O., Castagnoli, F., Venturi, V., and Morandi, M.: Use of polarimetric lidar for the study of oriented ice plates in clouds, Appl. Optics, 45, 4878-4887, doi:10.1364/AO.45.004878, 2006.

Di, H., Hua, D., Yan, L., Hou, X., and Wei, X.: Polarization analysis and corrections of different telescopes in polarization lidar, Appl. Optics, 54, 389-397, 2015.

Eloranta, E.: High Spectral Resolution Lidar, Springer New York, 143-163, 2005.

Eloranta, E.W. and Piironen, P.: Depolarization measurements with the High Spectral Resolution Lidar, Seventeenth International Laser Radar Conference, Sendai, Japan, 1994.

Engelmann, R., Kanitz, T., Baars, H., Heese, B., Althausen, D., Skupin, A., Wandinger, U., Komppula, M., Stachlewska, I. S., Amiridis, V., Marinou, E., Mattis, I., Linn., and Ansmann, A.: The automated multiwavelength Raman polarization and watervapor lidar PollyXT: the neXT generation, Atmos. Meas. Tech., 9, 1767-1784, doi:10.5194/amt-9-1767-2016, 2016.

Esselborn, M., Wirth, M., Fix, A., Tesche, M., and Ehret, G.: Airborne high spectral resolution lidar for measuring aerosol extinction and backscatter coefficients, Appl. Optics, 47, 346-358, 2008.

Flynn, C. J., Mendoza, A., Zheng, Y., and Mathur, S.: Novel polarization-sensitive micropulse lidar measurement technique, Opt. Express, 15, 2785-2790, 2007.

Freudenthaler, V., Esselborn, M., Wiegner, M., Heese, B., Tesche, M., Ansmann, A., Müller, D., Althausen, D., Wirth, M., Fix, A., Ehret, G., Knippertz, P., Toledano, C., Gasteiger, J., Garhammer, M., and Seefeldner, M.: Depolarization ratio profiling at several wavelengths in pure Saharan dust during SAMUM 2006, Tellus B, 61, 165-179, 2009.

Freudenthaler, V., Seefeldner, M., Gro, S., and Wandinger, U.: Accuracy of linear depolaristion ratios in clear air ranges measured with POLIS-6 at 355 and 532 nm, in: 27th International Laser Radar Conference, 2015.

Gasteiger, J. and Freudenthaler, V.: Benefit of depolarization ratio at $\lambda=1064 \mathrm{~nm}$ for the retrieval of the aerosol microphysics from lidar measurements, Atmos. Meas. Tech., 7, 3773-3781, doi:10.5194/amt-7-3773-2014, 2014.

Gasteiger, J., Wiegner, M., Groß, S., Freudenthaler, V., Toledano, C., Tesche, M., and Kandler, K.: Modelling lidarrelevant optical properties of complex mineral dust aerosols, Tellus B, 63, 725-741, 2011.

Geier, M. and Arienti, M.: Detection of preferential particle orientation in the atmosphere: Development of an alternative polarization lidar system, J. Quant. Spectrosc. Ra., 149, 16-32, 2014.

Gimmestad, G. G.: Reexamination of depolarization in lidar measurements, Appl. Optics, 47, 3795-3802, 2008.

Goldstein, D.: Polarized Light, Marcel Dekker, 2003.

Groß, S., Freudenthaler, V.,Wirth, M., andWeinzierl, B.: Towards an aerosol classification scheme for future EarthCARE lidar observations and implications for research needs, Atmos. Sci. Lett., 16, 77-82, doi:10.1002/as12.524, 2014.

Hair, J. W., Hostetler, C. A., Cook, A. L., Harper, D. B., Ferrare, R. A., Mack, T. L., Welch, W., Izquierdo, L. R., and Hovis, F. E.: Airborne High Spectral Resolution Lidar for profiling aerosol optical properties, Appl. Optics, 47, 6734$6752,2008$.

Handbook of Optics, Volume I - Geometrical and Physical Optics, Polarized Light, Components and Instruments, 3rd Edn., edited by: Bass, M., McGraw Hill Professional, 2009. 
Hauge, P., Muller, R., and Smith, C.: Conventions and formulas for using the Mueller-Stokes calculus in ellipsometry, Surf. Sci., 96, 81-107, 1980.

Hayman, M.: Optical Theory for the Advancement of Polarization Lidar, PhD thesis, University of Colorado at Boulder, ECEE dept., 2011.

Hayman, M., Spuler, S., and Morley, B.: Polarization lidar observations of backscatter phase matrices from oriented ice crystals and rain, Opt. Express, 22, 16976-16990, 2014.

Hayman, M., Spuler, S., Morley, B., and VanAndel, J.: Polarization lidar operation for measuring backscatter phase matrices of oriented scatterers, Opt. Express, 20, 29553-29567, 2012.

Hayman, M. and Thayer, J. P.: Explicit description of polarization coupling in lidar applications, Opt. Lett., 34, 611613, 2009.

Hayman, M. and Thayer, J. P.: General description of polarization in lidar using Stokes vectors and polar decomposition of Mueller matrices, J. Opt. Soc. Am. A, 29, 400-409, 2012.

Houston, J. D. and Carswell, A. I.: Four-component polarization measurement of lidar atmospheric scattering, Appl. Optics, 17, 614-620, 1978 .

Hunt,W. H.,Winker, D. M., Vaughan, M. A., Powell, K. A., Lucker, P. L. and Weimer, C.: CALIPSO Lidar Description and Performance Assessment, J. Atmos. Ocean. Tech., 26, 1214-1228, 2009.

Kahnert, M., Nousiainen, T., and Lindqvist, H.: Review: Model particles in atmospheric optics, J. Quant. Spectr. Ra., 146, 41-58, 2014.

Kaul, B. V., Kuznetsov, A. L., and Polovtseva, E. R.: Measurements of backscattering phase matrices of crystalline clouds with a polarization lidaR, Atmospheric and Oceanic Optics, 5, 381-383, 1992.

Kaul, B. V., Samokhvalov, I. V. and Volkov, S. N.: Investigating Particle Orientation in Cirrus Clouds by Measuring Backscattering Phase Matrices with Lidar, Appl. Optics, 43, 6620-6628, 2004.

Kölbl, C.: Depolarization of lidar signals in the arctic atmosphere; Calibration and optimization of the ALOMAR Troposphere Lidar, Bachelor thesis, University of Constance, Department of Physics, Germany, 69 pp., 2010.

Liu, Z., McGill, M., Hu, Y., Hostetler, C., Vaughan, M., and Winker, D.: Validating Lidar Depolarization Calibration Using Solar Radiation Scattered by Ice Clouds, IEEE Geosci. Remote Sens. Lett., 1, 157-161, 2004.

Lu, S.-Y. and Chipman, R. A.: Interpretation of Mueller matrices based on polar decomposition, J. Opt. Soc. Am. A, 13, 1106-1113, 1996.

Mattis, I., Tesche, M., Grein, M., Freudenthaler, V., and Müller, D.: Systematic error of lidar profiles caused by a polarizationdependent receiver transmission: quantification and error correction scheme, Appl. Optics, 48, 2742-2751, 2009.

McGill, M., Hlavka, D., Hart, W., Scott, V. S., Spinhirne, J., and Schmid, B.: Cloud Physics Lidar: instrument description and initial measurement results, Appl. Optics, 41, 3725-3734, 2002.

Mishchenko, M., Travis, L., and Lacis, A.: Scattering, absorption, and emission of light by small particles, Cambridge University Press, 2002.

Mishchenko, M. I. and Hovenier, J. W.: Depolarization of light backscattered by randomly oriented nonspherical particles, Opt. Lett., 20, 1356-1358, 1995. 
Müller, D., Hostetler, C. A., Ferrare, R. A., Burton, S. P., Chemyakin, E., Kolgotin, A., Hair, J. W., Cook, A. L., Harper, D. B., Rogers, R. R., Hare, R. W., Cleckner, C. S., Obland, M. D., Tomlinson, J., Berg, L. K., and Schmid, B.: Airborne Multiwavelength High Spectral Resolution Lidar (HSRL-2) observations during TCAP 2012: vertical profiles of optical and microphysical properties of a smoke/urban haze plume over the northeastern coast of the US, Atmos. Meas. Tech., 7, 3487-3496, doi:10.5194/amt-7-3487-2014, 2014.

Müller, D., Wandinger, U., and Ansmann, A.: Microphysical Particle Parameters from Extinction and Backscatter Lidar Data by Inversion with Regularization: Theory, Appl. Optics, 38, 2346-2357, 1999.

Muller, R.: Definitions and conventions in ellipsometry, Surf. Sci., 16, 14-33, 1969.

Nee, S.-M. F.: Errors of Mueller matrix measurements with a partially polarized light source, Appl. Optics, 45, 64976506, 2006.

Nemuc, A., Vasilescu, J., Talianu, C., Belegante, L., and Nicolae, D.: Assessment of aerosol's mass concentrations from measured linear particle depolarization ratio (vertically resolved) and simulations, Atmos. Meas. Tech., 6, 32433255, doi:10.5194/amt-6-3243-2013, 2013.

Nisantzi, A., Mamouri, R. E., Ansmann, A., and Hadjimitsis, D.: Injection of mineral dust into the free troposphere during fire events observed with polarization lidar at Limassol, Cyprus, Atmos. Chem. Phys., 14, 12155-12165, doi:10.5194/acp-14-12155-2014, 2014.

Nousiainen, T., Kahnert, M., and Lindqvist, H.: Can particle shape information be retrieved from light-scattering observations using spheroidal model particles?, J. Quant. Spectr. Ra., 112, 2213-2225, 2011.

Pal, S. R. and Carswell, A. I.: Polarization Properties of Lidar Backscattering from Clouds, Appl. Optics, 12, 15301535, 1973.

Pérez-Ramírez, D., Whiteman, D. N., Veselovskii, I., Kolgotin, A., Korenskiy, M., and Alados-Arboledas, L.: Effects of systematic and random errors on the retrieval of particle microphysical properties from multiwavelength lidar measurements using inversion with regularization, Atmos. Meas. Tech., 6, 3039-3054, doi:10.5194/amt-6-3039-2013, 2013.

Pezzaniti, L. J. and Chipman, R. A.: Angular dependence of polarizing beam splitter cubes, Appl. Optics, 33, 19161929, 1994.

Platt, C. M. R.: Lidar Observation of a Mixed-Phase Altostratus Cloud, J. Appl. Meteor., 16, 339-345, 1977.

Reichardt, J., Baumgart, R., and McGee, T. J.: Three-Signal Method for Accurate Measurements of Depolarization Ratio with Lidar, Appl. Optics, 42, 4909-4913, 2003.

Roy, G., Cao, X., and Bernier, R.: On the information content of linear and circular depolarization signatures of bioaerosols, in: Proc. SPIE 8018, Chemical, Biological, Radiological, Nuclear, and Explosives (CBRNE) Sensing XII, 801807, doi:10.1117/12.883713, 3 June 2011.

Sassen, K.: The Polarization Lidar Technique for Cloud Research: A Review and Current Assessment, B. Am. Meteorol. Soc., 72, 1848-1866, 1991.

Sassen, K.: Polarization in lidar, in: Lidar: Range-Resolved Optical Remote Sensing of the Atmosphere, Springer, 1942, 2005.

Sassen, K. and Benson, S.: A Midlatitude Cirrus Cloud Climatology from the Facility for Atmospheric Remote Sensing. Part II: Microphysical Properties Derived from Lidar Depolarization, J. Atmos. Sci., 58, 2103-2112, 2001. 
Schotland, R. M., Sassen, K., and Stone, R.: Observations by Lidar of Linear Depolarization Ratios for Hydrometeors, J. Appl. Meteorol., 10, 1011-1017, 1971.

Seldomridge, N. L., Shaw, J. A., and Repasky, K. S.: Dualpolarization lidar using a liquid crystal variable retarder, Opt. Eng., 45, 106202, doi:10.1117/1.2358636, 2006.

Shimizu, A., Sugimoto, N., Matsui, I., Arao, K., Uno, I., Murayama, T., Kagawa, N., Aoki, K., Uchiyama, A., and Yamazaki, A.: Continuous observations of Asian dust and other aerosols by polarization lidars in China and Japan during ACE-Asia, J. Geophys. Res.-Atmos., 109, doi:10.1029/2002JD003253, 2004.

Skumanich, A., Lites, B. W., Pillet, V. M., and Seagraves, P.: The Calibration of the Advanced Stokes Polarimeter, The Astrophysical Journal Supplement Series, 110, 357-380, doi:10.1086/313004, 1997.

Snels, M., Cairo, F., Colao, F., and Di Donfrancesco, G.: Calibration method for depolarization lidar measurements, Int. J. Remote Sens., 30, 5725-5736, 2009.

Socas-Navarro, H., Elmore, D., Asensio Ramos, A., and Harrington, D. M.: Characterization of telescope polarization properties across the visible and near-infrared spectrum, Astronomy Astrophysics, 531, A2, 8 pp., doi:10.1051/00046361/201015804, 2011.

Spinhirne, J. D., Hansen, M. Z., and Caudill, L. O.: Cloud top remote sensing by airborne lidar, Appl. Optics, 21, 1564$1571,1982$.

Steinborn, E. and Ruedenberg, K.: Rotation and Translation of Regular and Irregular Solid Spherical Harmonics, in: Advances in Quantum Chemistry 7, Löwdin, P.-O. (Ed.), Academic Press, 1-81, doi:10.1016/S0065-3276(08)60558-4, 1973.

Sugimoto, N. and Lee, C. H.: Characteristics of dust aerosols inferred from lidar depolarization measurements at two wavelengths, Appl. Optics, 45, 7468-7474, 2006.

Sugimoto, N., Matsui, I., Shimizu, A., Uno, I., Asai, K., Endoh, T., and Nakajima, T.: Observation of dust and anthropogenic aerosol plumes in the Northwest Pacific with a two-wavelength polarization lidar on board the research vessel Mirai, Geophys. Res. Lett., 29, 1901, doi:10.1029/2002GL015112, 2002.

van de Hulst, H. C.: Light scattering by small particles, Dover Publications, New York, 1981.

Veselovskii, I., Whiteman, D. N., Korenskiy, M., Kolgotin, A., Dubovik, O., Perez-Ramirez, D., and Suvorina, A.: Retrieval of spatio-temporal distributions of particle parameters from multiwavelength lidar measurements using the linear estimation technique and comparison with AERONET, Atmos. Meas. Tech., 6, 2671-2682, doi:10.5194/amt-62671-2013, 2013.

Volkov, S. N., Samokhvalov, I. V., Cheong, H. D., and Kim, D.: Investigation of East Asian clouds with polarization light detection and ranging, Appl. Optics, 54, 3095-3105, 2015.

Winker, D. M., Vaughan, M. A., Omar, A., Hu, Y., Powell, K. A., Liu, Z., Hunt, W. H., and Young, S. A.: Overview of the CALIPSO Mission and CALIOP Data Processing Algorithms, J. Atmos. Ocean. Tech., 26, 2310-2323, 2009. 